\title{
KNOT DOUBLING OPERATORS AND BORDERED HEEGAARD FLOER HOMOLOGY
}

\author{
ADAM SIMON LEVINE
}

\begin{abstract}
We use bordered Heegaard Floer homology to compute the $\tau$ invariant of a family of satellite knots obtained via twisted infection along two components of the Borromean rings, a generalization of Whitehead doubling. We show that $\tau$ of the resulting knot depends only on the two twisting parameters and the values of $\tau$ for the two companion knots. We also include some notes on bordered Heegaard Floer homology that may serve as a useful introduction to the subject.
\end{abstract}

\section{Contents}

1. Introduction

2. Background on bordered Heegaard Floer homology

2.1. Algebraic objects

2.2. The torus algebra

2.3. Bordered 3-manifolds and their invariants

2.4. Bimodules

2.5. Knots in bordered manifolds

2.6. The edge reduction algorithm

2.7. $\widehat{\mathrm{CFD}}$ of knot complements

3. Direct computation of $\widehat{\mathrm{CFAA}}\left(Y, B_{3}\right)$

3.1. A Heegaard diagram for $\left(\mathcal{Y}, B_{3}\right)$

3.2. Enumerating index-1 positive domains

3.3. Algebraic computation of $\widehat{\mathrm{CFA} A}$

4. Evaluation of the tensor product

4.1. Tensor product over $\mathcal{A}_{\rho}$

4.2. Simplification of $\widehat{\mathrm{CFA}}\left(\mathcal{V}, D_{J, s}\right)$

4.3. Tensor product over $\mathcal{A}_{\sigma}$

4.4. Computation of $\tau\left(D_{J, s}(K, t)\right)$

5. Other results regarding $D_{J, s}(K, t)$

Appendix A. Notes on the computation

A.1. HeegaardDiagram.nb

A.2. TorusAlgebra.nb

A.3. Borromean.nb

The author was supported by NSF grants DMS-0739392 and DMS-1004622. This paper appears in Journal of Topology $\mathbf{5}$ (2012) 651-712. 

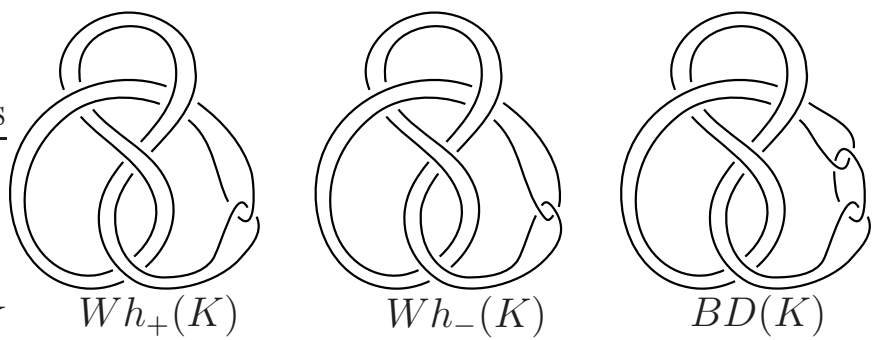

Figure 1. The positive and negative Whitehead doubles and the Bing double of the figure-eight knot.

\section{INTRODUCTION}

A knot in the 3-sphere is called topologically slice if it bounds a locally flatly embedded disk in the 4-ball, and smoothly slice if the disk can be taken to be smoothly embedded. Two knots are called (topologically or smoothly) concordant if they are the ends of an embedded annulus in $S^{3} \times I$; thus, a knot is slice if and only if it is concordant to the unknot. More generally, a link is (topologically or smoothly) slice if it bounds a disjoint union of appropriately embedded disks. The study of concordance - especially the relationship between the notions of topological and smooth sliceness - is one of the major areas of active research in knot theory, and it is closely tied to the perplexing differences between topological and smooth 4-manifold theory.

While all known explicit constructions of slice disks use smooth techniques, the early obstructions to sliceness - including the Alexander polynomial, the signature, J. Levine's algebraic concordance group, and Casson-Gordon invariants - arise from the algebraic topology of the complement of a slice disk, so they only obstruct a knot from being topologically slice. However, in the 1980s, Freedman [3] showed that any knot whose Alexander polynomial is 1 is topologically slice, even though it is difficult to describe the slice disks explicitly. In particular, the untwisted positive and negative Whitehead doubles of any knot $K$, denoted $W h_{ \pm}(K)$ (Figure 1), are topologically slice. Around the same time, the advent of Donaldson's gauge theory made it possible to show that some of these examples are not smoothly slice. Akbulut [unpublished] first proved in 1983 that the positive, untwisted Whitehead double of the right-handed trefoil is not smoothly slice. Later, using results of Kronheimer and Mrowka on Seiberg-Witten theory, Rudolph [20] showed that any nontrivial knot that is strongly quasipositive cannot be smoothly slice. In particular, the positive, untwisted Whitehead double of a strongly quasipositive knot is strongly quasipositive; thus, by induction, any iterated positive Whitehead double of a strongly quasipositive knot is topologically but not smoothly slice. Bižaca [1] used this result to give explicit constructions of exotic smooth structures on $\mathbb{R}^{4}$.

Using Heegaard Floer homology, Ozsváth and Szabó [14] defined an additive, integer-valued knot invariant $\tau(K)$, defined as the minimum Alexander grading of an element of $\widehat{\operatorname{HFK}}\left(S^{3}, K\right)$ that survives to the $E^{\infty}$ page of the spectral sequence from $\widehat{\operatorname{HFK}}\left(S^{3}, K\right)$ to $\widehat{\mathrm{HF}}\left(S^{3}\right)$. The $\tau$ invariant provides a lower bound on the genus 
(a)

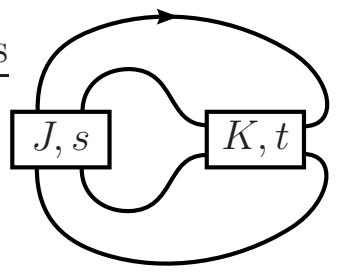

(b)

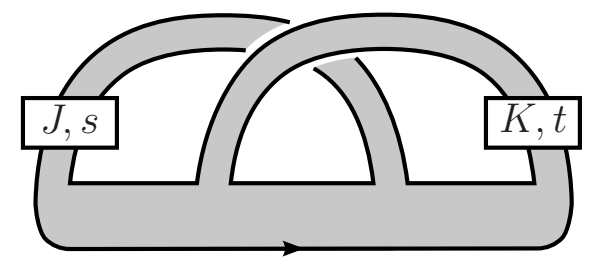

Figure 2. (a) The knot $D_{J, s}(K, t)$. (b) A genus-1 Seifert surface for $D_{J, s}(K, t)$.

of smooth surfaces in the four-ball bounded by $K:|\tau(K)| \leq g_{4}(K)$. In particular, if $K$ is smoothly slice, then $\tau(K)=0$. This fact can be used to generalize many of the previously known results about knots that are topologically but not smoothly slice. For example, Hedden [4] computed the value of $\tau$ for all twisted Whitehead doubles in terms of $\tau$ of the original knot:

$$
\tau\left(W h_{+}(K, t)\right)= \begin{cases}1 & t<2 \tau(K) \\ 0 & t \geq 2 \tau(K) .\end{cases}
$$

(An analogous formula for negative Whitehead doubles follows from the fact that $\tau(\bar{K})=-\tau(K)$.) In particular, if $\tau(K)>0$, then $\tau\left(W h_{+}(K, 0)\right)=1$, so $W h_{+}(K, 0)$ (the untwisted Whitehead double of $K$ ) is not smoothly slice. Since $\tau$ of a strongly quasipositive knot is equal to its genus [12], Rudolph's result follows from Hedden's. There is a famous conjecture (Problem 1.38 on Kirby's problem list [8]) that the untwisted Whitehead double of $K$ is smoothly slice if and only if $K$ is smoothly slice. However, it is not yet known whether, for instance, the positive Whitehead double of the left-handed trefoil is smoothly slice. Indeed, it seems that gauge theory invariants have a fundamental asymmetry that makes them unable to detect such examples, which likely places the "only if" direction of this conjecture beyond the scope of currently existing techniques.

We consider the following generalization of Whitehead doubling. For knots $J, K$ and integers $s, t$, let $D_{J, s}(K, t)$ denote the knot shown in Figure2(a); the box marked $K, t$ (resp. $J, s$ ) indicates that the strands are tied along $t$-framed (resp. $s$-framed) parallel copies of the tangle $K \backslash\{\mathrm{pt}\}$ (resp. $J \backslash\{\mathrm{pt}\}$ ). (We give a more formal definition below.) If $J$ is the unknot and $s= \pm 1$, then $D_{J, s}(K, t)$ is the $t$-twisted $\mp$ Whitehead double of $K$.

A genus-1 Seifert surface for $D_{J, s}(K, t)$ is shown in Figure 2(b). From the Seifert form of this surface, we can compute that the Alexander polynomial of $D_{J, s}(K, t)$ is

$$
\Delta_{D_{J, s}(K, t)}(T)=s t T+(1-2 s t)+s t T^{-1} \text {. }
$$

In particular, this equals 1 whenever $s=0$ or $t=0$. By Freedman's theorem, $D_{J, s}(K, 0)$ is therefore topologically slice. Moreover, if $K$ is smoothly slice, then $D_{J, s}(K, 0)$ is smoothly slice for any $(J, s)$. To see this, perform a ribbon move to eliminate the band that is tied into $J$; the resulting two-component link, consisting of two parallel copies of $K$ with linking number 0 , is then the boundary of two parallel 


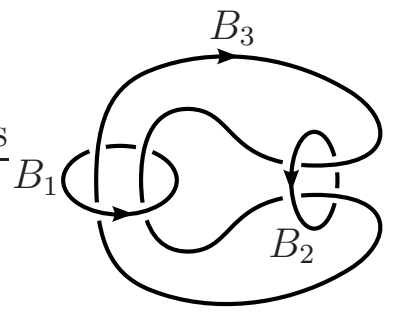

Figure 3. The Borromean rings.

copies of a slice disk for $K$. The conjecture about sliceness of untwisted Whitehead doubles described above has many potential generalizations in terms of $D_{J, s}(K, 0)$ satellites, all apparently equally difficult.

As a partial result in this direction, we prove the following theorem, which generalizes Hedden's result:

Theorem 1.1. Let $J$ and $K$ be knots, and let $s, t \in \mathbb{Z}$. Then

$$
\tau\left(D_{J, s}(K, t)\right)= \begin{cases}1 & s<2 \tau(J) \text { and } t<2 \tau(K) \\ -1 & s>2 \tau(J) \text { and } t>2 \tau(K) \\ 0 & \text { otherwise. }\end{cases}
$$

In particular, if $\tau(K)>0$ and $s<2 \tau(J)$, or if $\tau(K)<0$ and $s>2 \tau(J)$, then $D_{J, s}(K, 0)$ is topologically but not smoothly slice.

We now provide a more rigorous description of $D_{J, s}(K, t)$. Suppose $L$ is a link in $S^{3}$, and $\gamma$ is an oriented curve in $S^{3} \backslash L$ that is unknotted in $S^{3}$. For any knot $K \subset S^{3}$ and $t \in \mathbb{Z}$, we may form a new link $I_{\gamma, K, t}(L)$, the $t$-twisted infection of $L$ by $K$ along $\gamma$, by deleting a neighborhood of $\gamma$ and gluing in a copy of the exterior of $K$ by a map that takes a Seifert-framed longitude of $K$ to a meridian of $\gamma$ and a meridian of $K$ to a $t$-framed longitude of $\gamma$. Since $S^{3} \backslash \gamma=S^{1} \times D^{2}$, the resulting 3-manifold is simply $\infty$ surgery on $K$, i.e. $S^{3}$; the new link $I_{\gamma, K, t}(L)$ is defined as the image of $L$. Infecting along the boundary of a disk perpendicular to a group of strands formalizes the notion of "tying the strands into a knot." Moreover, given an unlink $\gamma_{1}, \ldots, \gamma_{n}$ disjoint from $L$, we may infect simultaneously along all the $\gamma_{i}$; the result may be denoted $I_{\gamma_{1}, K_{1}, t_{1} ; \cdots ; \gamma_{n}, K_{n}, t_{n}}(L)$, and the order of the tuples $\left(\gamma_{i}, K_{i}, t_{i}\right)$ does not matter.

Let $B=B_{1} \cup B_{2} \cup B_{3}$ denote the Borromean rings, oriented as shown in Figure 3 . Then $D_{J, s}(K, t)$ is the knot obtained from $B_{3}$ by $s$-twisted infection by $J$ along $B_{1}$ and $t$-twisted infection by $K$ along $B_{2}$ :

$$
D_{J, s}(K, t)=I_{B_{1}, J, s ; B_{2}, K, t}\left(B_{3}\right) .
$$

The theory of bordered Heegaard Floer homology, developed recently by Lipshitz, Ozsváth, and Thurston [10, 11], is well-suited to the problem of computing Heegaard Floer invariants of knots obtained via infection. Briefly, it associates to a 3-manifold with boundary a module over an algebra associated to the boundary, so that if a 3manifold $Y$ is decomposed as $Y=Y_{1} \cup_{\phi} Y_{2}$, where $\phi: \partial Y_{1} \cong \partial Y_{2}$, the chain complex $\widehat{\mathrm{CF}}(Y)$ may be computed as the derived tensor product of the invariants associated 
to $Y_{1}$ and $Y_{2}$. If a knot $K$ is contained (nulhomologously) in, say, $Y_{1}$, then we may obtain the filtration on $\widehat{\mathrm{CF}}(Y)$ corresponding to $K$ via a filtration on the algebraic invariant of $Y_{1}$. The theory also includes bimodules associated to manifolds with two boundary components.

In our setting, let $Y$ denote the exterior of $B_{1} \cup B_{2}$, and let $X_{J}$ and $X_{K}$ denote the exteriors of $J$ and $K$, respectively. For suitable gluing maps $\phi_{1}: \partial X_{J} \rightarrow \partial_{1} Y$ and $\phi_{2}: \partial X_{K} \rightarrow \partial_{2} Y$ (where $\left.\partial Y=\partial_{1} Y \amalg \partial_{2} Y\right)$, the glued-up manifold $\left(Y \cup_{\phi_{1}} X_{J}\right) \cup_{\phi_{2}} X_{K}$ is $S^{3}$, and the image of $B_{3} \subset Y$ is $D_{J, s}(K, t)$. We shall define suitable bordered structures $\mathcal{Y}, \mathcal{X}_{J}^{s}$, and $\mathcal{X}_{K}^{t}$ on $Y, X_{J}$, and $X_{K}$, respectively, so as to induce these gluing maps. By the gluing theorem of Lipshitz, Ozsváth, and Thurston, the filtered chain complex for $\left(S^{3}, D_{J, s}(K, t)\right)$ can then computed as a special tensor product of the modules associated to $\mathcal{Y}, \mathcal{X}_{J}^{s}$, and $\mathcal{X}_{K}^{t}$ :

$$
\widehat{\mathrm{CF}}\left(S^{3}, D_{J, s}(K, t)\right) \simeq\left(\widehat{\mathrm{CFAA}}\left(\mathcal{Y}, B_{3}, 0\right) \otimes \widehat{\mathrm{CFD}}\left(\mathcal{X}_{J}^{s}\right)\right) \otimes \widehat{\mathrm{CFD}}\left(\mathcal{X}_{K}^{t}\right)
$$

All of this terminology will be explained in Section 2. Using the formula for $\widehat{\mathrm{CFD}}\left(\mathcal{X}_{J}^{s}\right)$ and $\widehat{\mathrm{CFD}}\left(\mathcal{X}_{K}^{t}\right)$ proven by Lipshitz, Ozsváth, and Thurston [10] and a direct computation of $\widehat{\operatorname{CFAA}}\left(\mathcal{Y}, B_{3}, 0\right)$ using holomorphic disks (given in Section 3), we shall explicitly evaluate this double tensor product and compute its homology in Section 4 , leading to the proof of Theorem 1.1. While the proof is fairly technical, it illustrates the power of the new bordered techniques: using a single computation involving holomorphic disks (which can in principle be performed entirely combinatorially) and some lengthy but straightforward algebra, we are able to obtain a statement about the Floer homology an infinite family of knots. The proof relies on some computer-assisted computation using Mathematica; the details are described in Appendix A.

In Section 5, we present a few more results concerning knots of the form $D_{J, s}(K, t)$. Following the approach of Livingston and Naik [13, we show that if $\nu$ is any concordance invariant that shares certain formal properties with $\tau$ - e.g., Rasmussen's $s$ invariant coming from Khovanov homology - then $\nu\left(D_{J, s}(K, t)\right)=\tau\left(D_{J, s}(K, t)\right)$ when $|s|$ and $|t|$ are sufficiently large. We also provide a family of examples of knots of the form $D_{J, s}(K, t)$ that are smoothly slice, generalizing a result of Casson about Whitehead doubles.

Finally, Theorem 1.1 has a useful application to the study of Whitehead doubles of links, which was the author's original motivation for considering it. Specifically, we consider the Whitehead doubles of links obtained by iterated Bing doubling. Given a knot $K$, the (untwisted) Bing double of $K$ is the satellite link $B D(K)=I_{B_{1}, K, 0}\left(B_{2} \cup\right.$ $B_{3}$ ), as shown in Figure 1. More generally, given a link $L$, we may replace a component by its Bing double (contained in a tubular neighborhood of that component), and iterate this procedure. Bing doubling one component of the Hopf link yields the Borromean rings; accordingly, we define the family of generalized Borromean links as the set of all links obtained as iterated Bing doubles of the Hopf link. Using Theorem 1.1, the author proves in [9]:

Corollary 1.2. Let $L$ be any link obtained by iterated Bing doubling from either:

(1) Any knot $K$ with $\tau(K)>0$, or 
(2) The Hopf link.

Then the all-positive Whitehead double of $L, W h_{+}(L)$, is not smoothly slice.

The links in (1) are boundary links, so their Whitehead doubles are all topologically slice by a result of Freedman [2]. On the other hand, it is not yet known whether the Whitehead doubles of iterated Bing doubles of the Hopf link are topologically slice; indeed, this question is one of the major unsolved problems in four-dimensional topological surgery theory. Once again, we see a strong dependence on chirality; our proof breaks down when clasps of both signs are used. For further details, see [9].

Acknowledgements. A version of this paper made up a large portion of the author's thesis at Columbia University. The author is grateful to his advisor, Peter Ozsváth, and the other members of his defense committee, Robert Lipshitz, Dylan Thurston, Paul Melvin, and Denis Auroux, for their suggestions, and to Rumen Zarev, Ina Petkova, Jen Hom, and Matthew Hedden for many helpful conversations about bordered Heegaard Floer homology. Additionally, he thanks the Mathematical Sciences Research Institute for hosting him in Spring 2010, when much of this research was conducted.

\section{Background on Bordered HeEgaArd Floer homology}

In this section, we give a brief description of the bordered Heegaard Floer invariants, with the aim of defining the terms used later in the paper and illustrating the procedures for computation. We discuss only bordered manifolds with toroidal boundary components, which has the advantage of greatly simplifying some of the definitions. All of this material can be found in the two magna opera of Lipshitz, Ozsváth, and Thurston [10, 11].

2.1. Algebraic objects. We first recall the main algebraic constructions used in [10, 11, with the aim of describing how to work with them computationally. Let $(\mathcal{A}, d)$ be a unital differential algebra over $\mathbb{F}=\mathbb{F}_{2}$, and assume that the set of $\mathcal{I}$ of idempotents in $\mathcal{A}$ is a commutative subring of $\mathcal{A}$ and possesses a basis $\left\{\iota_{i}\right\}$ over $\mathbb{F}$ such that $\iota_{i} \iota_{j}=\delta_{i j} \iota_{i}$ and $\sum_{i} \iota_{i}=1$, the identity element of $\mathcal{A}$. (All of the definitions that follow can be stated in terms of differential graded algebras, but we suppress all grading information for brevity.)

- A (right) $\mathcal{A}_{\infty}$ module or type $A$ structure over $\mathcal{A}$ is an $\mathbb{F}$-vector space $M$, equipped with a right action of $\mathcal{I}$ such that $M=\bigoplus_{i} M \iota_{i}$ as a vector space, and multiplication maps

$$
m_{k+1}: M \underset{\mathcal{I}}{\otimes} \underbrace{\mathcal{A} \underset{\mathcal{I}}{\otimes} \ldots \underset{\mathcal{I}}{\otimes} \mathcal{A}}_{k \text { times }} \rightarrow M
$$


satisfying the $\mathcal{A}_{\infty}$ relations: for any $x \in M$ and $a_{1}, \ldots, a_{n} \in \mathcal{A}$,

$$
\begin{aligned}
0 & =\sum_{i=0}^{n} m_{n-i+1}\left(m_{i+1}\left(x, a_{1}, \ldots, a_{i}\right), a_{i+1}, \ldots, a_{n}\right) \\
& +\sum_{i=1}^{n} m_{n+1}\left(x, a_{1}, \ldots, a_{i-1}, d\left(a_{i}\right), a_{i+1}, \ldots, a_{n}\right) \\
& +\sum_{i=1}^{n-1} m_{n}\left(x, a_{1}, \ldots, a_{i-1}, a_{i} a_{i+1}, a_{i+2}, \ldots, a_{n}\right) .
\end{aligned}
$$

We also require that $m_{2}(x, \mathbf{1})=x$ and $m_{k}(x, \ldots, \mathbf{1}, \ldots)=0$ for $k>2$.

The module $M$ is called bounded if $m_{k}=0$ for all $k$ sufficiently large. If $M$ is a bounded type $A$ structure with basis $\left\{x_{1}, \ldots, x_{n}\right\}$, we encode the multiplications using a matrix whose entries are formal sums of finite sequences of elements of $\mathcal{A}$, where having an $\left(a_{1}, \ldots, a_{k}\right)$ term in the $i, j^{\text {th }}$ entry means that the coefficient of $x_{j}$ in $m_{k+1}\left(x_{i}, a_{1}, \ldots, a_{k}\right)$ is nonzero. We write 1 rather than an empty sequence to signify the $m_{1}$ multiplication. For brevity, we frequently write $a_{1} \cdots a_{k}$ rather than $\left(a_{1}, \ldots, a_{k}\right)$; in this context, concatenation is not interpreted as multiplication in the algebra $\mathcal{A}$.

- A (left) type $D$ structure over $\mathcal{A}$ is an $\mathbb{F}$-vector space $N$, equipped with a left action of $\mathcal{I}$ such that $N=\bigoplus_{i} \iota_{i} N$, and a map

$$
\delta_{1}: N \rightarrow A \underset{\mathcal{I}}{\otimes} N
$$

satisfying the relation

$$
\left(\mu \otimes \operatorname{id}_{N}\right) \circ\left(\operatorname{id}_{\mathcal{A}} \otimes \delta_{1}\right) \circ \delta_{1}+\left(d \otimes \mathrm{id}_{N}\right) \circ \delta_{1}=0,
$$

where $\mu: \mathcal{A} \otimes \mathcal{A} \rightarrow \mathcal{A}$ denotes the multiplication on $\mathcal{A}$.

If $N$ is a type $D$ structure, the tensor product $\mathcal{A} \underset{\mathcal{I}}{\otimes} N$ is naturally a left differential module over $\mathcal{A}$, with module structure given by $a \cdot(b \otimes x)=a b \otimes x$, and differential $\partial(a \otimes x)=a \cdot \delta_{1}(x)+d(a) \otimes x$. Condition (2.2) translates to $\partial^{2}=0$.

Given a type- $D$ module $N$, define maps

$$
\delta_{k}: N \rightarrow \underbrace{\mathcal{A} \underset{\mathcal{I}}{\otimes} \ldots \otimes_{\mathcal{I}}^{\otimes} \mathcal{A}}_{k \text { times }} \otimes_{\mathcal{I}}^{\otimes} N
$$

by $\delta_{0}=\operatorname{id}_{N}$ and $\delta_{k}=\left(\operatorname{id}_{\mathcal{A}^{\otimes k-1}} \otimes \delta_{1}\right) \circ \delta_{k-1}$. We say $N$ is bounded if $\delta_{k}=0$ for all $k$ sufficiently large.

Given a basis $\left\{y_{1}, \ldots, y_{n}\right\}$ for $N$, we may encode $\delta_{1}$ as an $n \times n$ matrix $\left(b_{i j}\right)$ with entries in $\mathcal{A}$, such that $\delta_{1} x_{i}=\sum_{j=1}^{n} b_{i j} \otimes x_{j}$. To encode $\delta_{k}$ in matrix form, we take the $k^{\text {th }}$ power of the matrix for $\delta_{1}$, except that instead of evaluating multiplication in $\mathcal{A}$, we simply concatenate tensor products of elements.

If $d=0,(2.2)$ is equivalent to the statement that the square of the matrix for $\delta_{1}$ (where now we do evaluate multiplication in $\mathcal{A}$ ) is zero. 
- If $M$ is a right type $A$ structure, $N$ is a left type $D$ structure, and at least one of them is bounded, we may form the box tensor product $M \otimes N$. As a vector space, this is $M \underset{\mathcal{I}}{\otimes} N$, with differential

$$
\partial^{\bigotimes}(x \otimes y)=\sum_{k=0}^{\infty}\left(m_{k+1} \otimes \operatorname{id}_{N}\right)\left(x \otimes \delta_{k}(y)\right) .
$$

Given matrix representations of the multiplications on $M$ and the $\delta_{k}$ maps on $N$, it is easy to write down the differential on $M \otimes N$ in terms of the basis $\left\{x_{i} \otimes y_{j}\right\}$.

- Now let $\left(\mathcal{A}, d_{\mathcal{A}}\right)$ and $\left(\mathcal{B}, d_{\mathcal{B}}\right)$ be differential algebras. Lipshitz, Ozsváth, and Thurston [11] define various types of $(\mathcal{A}, \mathcal{B})$-bimodules. We do not define these in full detail, but we mention some of the basic notions.

A type $D D$ structure is simply a type $D$ structure over the $\operatorname{ring} \mathcal{A} \otimes \mathbb{F}$. That is, the map $\delta_{1}$ outputs terms of the form $a \otimes b \otimes x$, where $a \in \mathcal{A}$ and $b \in \mathcal{B}$.

A type $A A$ structure consists of a vector space $M$ with multiplications

$$
m_{1, i, j}: M \otimes \mathcal{A}^{\otimes i} \otimes \mathcal{B}^{\otimes j} \rightarrow M,
$$

satisfying a version of the $\mathcal{A}_{\infty}$ relation (2.1). As above, all tensor products are taken over the rings of idempotents, $\mathcal{I}_{\rho} \subset \mathcal{A}$ and $\mathcal{I}_{\sigma} \subset \mathcal{B}$. Our notation differs a bit from that of [11] in that we think of both algebras as acting on the right.

A type DA structure is a vector space $N$ with maps

$$
\delta_{1}^{1+j}: N \otimes \mathcal{B}^{\otimes j} \rightarrow \mathcal{A} \otimes N
$$

satisfying an appropriate relation that combines (2.1) and (2.2). A type $A D$ structure is defined similarly, except that the roles of $\mathcal{A}$ and $\mathcal{B}$ are interchanged.

The box tensor product of two bimodules, or of a module and a bimodule, can be defined assuming at least one of the factors is bounded (in an appropriate sense). See [11, Subsection 2.3.2] for details.

- A filtration on a type $A$ structure $M$ is a filtration $\cdots \subseteq \mathcal{F}_{p} \subseteq \mathcal{F}_{p+1} \subseteq \ldots$ of $M$ as a vector space, such that $m_{k+1}\left(\mathcal{F}_{p} \otimes \mathcal{A}^{\otimes k}\right) \subseteq \mathcal{F}_{p}$. Similarly, a filtration on a type $D$ structure $N$ is a filtration of $N$ such that $\delta_{1}\left(\mathcal{F}_{p}\right) \subseteq \mathcal{A} \otimes \mathcal{F}_{p}$. It is easy to extend these definitions to the various types of bimodules. A filtration on $M$ or $N$ naturally induces a filtration on $M \otimes N$.

2.2. The torus algebra. The pointed matched circle for the torus, $\mathcal{Z}$, consists of an oriented circle $Z$, equipped with a basepoint $z \in Z$, a tuple $\mathbf{a}=\left(a_{1}, a_{2}, a_{3}, a_{4}\right)$ of points in $Z \backslash\{z\}$ (ordered according to the orientation on $Z \backslash\{z\}$ ), and the equivalence relation $a_{1} \sim a_{3}, a_{2} \sim a_{4}$. The genus-1, one-boundary-component surface $F^{\circ}(\mathcal{Z})$ is obtained by identifying $Z$ with the boundary of a disk $D$ and attaching 1-handles $h_{1}$ and $h_{2}$ that connect $a_{1}$ to $a_{3}$ and $a_{2}$ to $a_{4}$, respectively. By attaching a 2-handle along $\partial F^{\circ}(\mathcal{Z})$, we obtain the closed surface $F(\mathcal{Z})$. There is an orientationreversing involution $r: Z \rightarrow Z$ that fixes $z$, interchanges $a_{1}$ and $a_{4}$, and interchanges 
$a_{2}$ and $a_{3}$, which extends to a diffeomorphism $r: F(\mathcal{Z}) \rightarrow-F(\mathcal{Z})$ that interchanges $h_{1}$ and $h_{2}$.

The algebra $\mathcal{A}=\mathcal{A}(\mathcal{Z}, 0)$ is generated as a vector space over $\mathbb{F}$ by two idempotents $\iota_{0}, \iota_{1}$ and six Reeb elements $\rho_{1}, \rho_{2}, \rho_{3}, \rho_{12}, \rho_{23}, \rho_{123}$. For each sequence of consecutive integers $I=\left(i_{1}, \ldots, i_{k}\right) \subset\{1,2,3\}$, we have $\iota_{\left[i_{1}-1\right]} \rho_{I}=\rho_{I} \iota_{\left[i_{k}\right]}=\rho_{I}$, where $[j]$ denotes the residue of $j$ modulo 2 . The nonzero multiplications among the Reeb elements are: $\rho_{1} \rho_{2}=\rho_{12}, \rho_{2} \rho_{3}=\rho_{23}, \rho_{1} \rho_{23}=\rho_{12} \rho_{3}=\rho_{123}$. All other products are zero, as is the differential. Let $\mathcal{I}$ denote the subring of idempotents of $\mathcal{A}$; it is generated as a vector space by $\iota_{0}$ and $\iota_{1}$. The identity element is $\mathbf{1}=\iota_{0}+\iota_{1}$.

By abuse of notation, we identify $\rho_{1}$ with the oriented arc of $Z$ from $a_{1}$ to $a_{2}, \rho_{2}$ with the arc from $a_{2}$ to $a_{3}, \rho_{3}$ with the arc from $a_{3}$ to $a_{4}$, and $\rho_{12}, \rho_{23}$, and $\rho_{123}$ with the appropriate concatenations.

2.3. Bordered 3-manifolds and their invariants. A bordered 3-manifold with boundary $F(\mathcal{Z})$ consists of the data $\mathcal{Y}=\left(Y, \Delta, z^{\prime}, \phi\right)$, where $Y$ is an oriented 3manifold with a single boundary component, $\Delta$ is a disk in $\partial Y, z^{\prime} \in \partial \Delta$, and $\phi: F(\mathcal{Z}) \rightarrow \partial(Y)$ is a diffeomorphism taking $D$ to $\Delta$ and $z$ to $z^{\prime}$. The map $\phi$ is specified (up to isotopy fixing $\Delta$ pointwise) by the images of the core arcs of the two one-handles in $F^{\circ}(\mathcal{Z})$. We may analogously define a bordered 3-manifold with boundary $-F(\mathcal{Z})$. The diffeomorphism $r: F(\mathcal{Z}) \rightarrow-F(\mathcal{Z})$ provides a one-to-one correspondence between these two types of bordered manifolds.

A bordered 3-manifold $\mathcal{Y}$ may be presented by a bordered Heegaard diagram

$$
\mathcal{H}=\left(\Sigma,\left\{\alpha_{1}^{c}, \ldots, \alpha_{g-1}^{c}, \alpha_{1}^{a}, \alpha_{2}^{a}\right\},\left\{\beta_{1}, \ldots, \beta_{g}\right\}, z\right),
$$

where $\Sigma$ is a surface of genus $g$ with one boundary components, $\left\{\alpha_{1}^{c}, \ldots, \alpha_{g-1}^{c}\right\}$ and $\left\{\beta_{1}, \ldots, \beta_{g}\right\}$ are tuples of homologically linearly independent, disjoint circles in $\Sigma$, and $\alpha_{1}^{a}$ and $\alpha_{2}^{a}$ are properly embedded arcs that are disjoint from the $\alpha$ circles and linearly independent from them in $H_{1}(\Sigma, \partial \Sigma)$. If we identify $\left(\partial \Sigma, z, \partial \Sigma \cap\left(\alpha_{1}^{a} \cup \alpha_{2}^{a}\right)\right)$ with $\mathcal{Z}$ - where $\partial \Sigma$ is given the boundary orientation — we obtain a bordered 3manifold with boundary parametrized by $F(\mathcal{Z})$ by attaching handles along the $\alpha$ and $\beta$ circles. If instead we identify $\partial \Sigma$ with $-\mathcal{Z}$, we obtain a bordered 3 -manifold with boundary parametrized by $-F(\mathcal{Z})$.

Let $\mathfrak{S}(\mathcal{H})$ denote the set of unordered $g$-tuples of points $\mathbf{x}=\left\{x_{1}, \ldots, x_{g}\right\}$ such that each $\alpha$ circle and each $\beta$ circle contains exactly one point of $\mathbf{x}$ and each $\alpha$ arc contains at most one point of $\mathbf{x}$. Let $X(\mathcal{H})$ denote the $\mathbb{F}_{2}$-vector space spanned by $\mathfrak{S}(H)$.

For generators $\mathbf{x}, \mathbf{y} \in \mathfrak{S}(\mathcal{H})$, let $\pi_{2}(\mathbf{x}, \mathbf{y})$ denote the set of homology classes of maps $u: S \rightarrow \Sigma \times[0,1] \times[-2,2]$, where $S$ is a surface with boundary, taking $\partial S$ to

$$
\begin{aligned}
((\boldsymbol{\alpha} \times\{1\} \cup \boldsymbol{\beta} \times\{0\} \cup(\partial \Sigma \backslash z) \times[0,1]) & \times[-2,2]) \cup \\
& (\mathbf{x} \times[0,1] \times\{-2\}) \cup(\mathbf{y} \times[0,1] \times\{2\})
\end{aligned}
$$

and mapping to the relative fundamental homology class of $(\mathbf{x} \times[0,1] \times\{-2\}) \cup$ $(\mathbf{y} \times[0,1] \times\{2\})$. Each element $B \in \pi_{2}(\mathbf{x}, \mathbf{y})$ is determined by its domain, the projection of $B$ to $H_{2}(\Sigma, \boldsymbol{\alpha} \cup \boldsymbol{\beta} \cup \partial \Sigma ; \mathbb{Z})$. The group $H_{2}(\Sigma, \boldsymbol{\alpha} \cup \boldsymbol{\beta} \cup \partial \Sigma ; \mathbb{Z})$ is freely 
generated by the closures of the components of $\Sigma \backslash(\boldsymbol{\alpha} \cup \boldsymbol{\beta})$, which we call regions. The domain of any $B \in \pi_{2}(\mathbf{x}, \mathbf{y})$ satisfies the following conditions:

- The multiplicity of the region containing the basepoint $z$ is 01

- For each point $p \in \boldsymbol{\alpha} \cap \boldsymbol{\beta}$, if we identify an oriented neighborhood of $p$ with $\mathbb{R}^{2}$, taking $p$ to the origin and the $\alpha$ and $\beta$ segments containing $p$ to the $x$ and $y$-axes, respectively, and let $n_{1}(p), n_{2}(p), n_{3}(p)$, and $n_{4}(p)$ denote the multiplicities in $D$ of the regions in the four quadrants, then

$$
n_{1}(p)-n_{2}(p)+n_{3}(p)-n_{4}(p)= \begin{cases}1 & p \in \mathbf{x} \backslash \mathbf{y} \\ -1 & p \in \mathbf{y} \backslash \mathbf{x} \\ 0 & \text { otherwise }\end{cases}
$$

Conversely, any such domain represents some $B \in \pi_{2}(\mathbf{x}, \mathbf{y})$. Thus, finding the elements of $\pi_{2}(\mathbf{x}, \mathbf{y})$ is a simple matter of linear algebra. A homology class $B \in \pi_{2}(\mathbf{x}, \mathbf{y})$ is called positive if the regions in its domain all have non-negative multiplicity; only positive classes can support holomorphic representatives.

We shall describe only the invariant $\widehat{\mathrm{CFD}}$ here, since we do not compute $\widehat{\mathrm{CFA}}$ explicitly from a Heegaard diagram in this paper.

We identify the boundary of $\Sigma$ with $-\mathcal{Z}$. Assume that the $\alpha$ arcs are labeled so that $\alpha_{1}^{a} \cap \partial \Sigma=\left\{a_{1}, a_{3}\right\}$ and $\alpha_{2}^{a} \cap \partial \Sigma=\left\{a_{2}, a_{4}\right\}$.

Define a function $I_{D}: \mathfrak{S}(\mathcal{H}) \rightarrow\left\{\iota_{0}, \iota_{1}\right\}$ by

$$
I_{D}(\mathbf{x})= \begin{cases}\iota_{0} & \mathbf{x} \cap \alpha_{2}^{a} \neq \varnothing \\ \iota_{1} & \mathbf{x} \cap \alpha_{1}^{a} \neq \varnothing .\end{cases}
$$

Define a left action of $\mathcal{I}$ on $X(\mathcal{H})$ by $\iota_{i} \cdot \mathbf{x}=\delta\left(\iota_{i}, I_{D}(\mathbf{x})\right) \mathbf{x}$, where $\delta$ is the Kronecker delta.

For each of the oriented $\operatorname{arcs} \rho_{I} \subset \mathcal{Z}$, let $-\rho_{I}$ denote $\rho_{I}$ with its opposite orientation. (That is, $-\rho_{1}$ goes from $a_{2}$ to $a_{1}$, etc.) Given $\mathbf{x} \in \mathfrak{S}(\mathcal{H})$ and a sequence $\vec{\rho}=\left(-\rho_{I_{1}}, \ldots,-\rho_{I_{k}}\right)$, the pair $(\mathbf{x}, \vec{\rho})$ is called strongly boundary monotonic if the initial point of $-\rho_{I_{1}}$ is on the same $\alpha$ circle as $\mathbf{x}$, and for each $i>1$, the initial point of $-\rho_{I_{i}}$ and the final point of $-\rho_{I_{i-1}}$ are paired in $\mathcal{Z}$.

If $B \in \pi_{2}(\mathbf{x}, \mathbf{y})$ is a positive class, then $\partial^{\partial} B$ (the intersection of the domain of $B$ with the boundary of $\Sigma$ ) may be expressed (non-uniquely) as a sum of arcs $-\rho_{I_{i}}$. Specifically, we say that the pair $(B, \vec{\rho})$ is compatible if $(\mathbf{x}, \vec{\rho})$ is strongly boundary monotonic and $\partial^{\partial} B=\sum_{i=1}^{k}\left(-\rho_{I_{i}}\right)$. If $(B, \vec{\rho})$ is compatible, the index of $(B, \vec{\rho})$ is defined in [10, Definition 5.61] as

$$
\operatorname{ind}(B, \vec{\rho})=e(B)+n_{\mathbf{x}}(B)+n_{\mathbf{y}}(B)+|\vec{\rho}|+\iota(\vec{\rho}),
$$

where $e(B)$ is the Euler measure of $B ; n_{\mathbf{x}}(B)$ (resp. $\left.n_{\mathbf{y}}(B)\right)$ is the sum over points $x_{i} \in \mathbf{x}$ (resp. $y_{i} \in \mathbf{y}$ ) of the average of the multiplicities of the regions incident to $x_{i}$ (resp. $\left.y_{i}\right),|\vec{\rho}|$ is the number of entries in $\vec{\rho}$, and $\iota(\vec{\rho})$ is a combinatorially defined quantity [10, Equation 5.58] that measures the overlapping of the arcs $\rho_{I_{i}}$. The index $\operatorname{ind}(B, \vec{\rho})$ is equal to one plus the expected dimension of a certain moduli space

\footnotetext{
${ }^{1}$ In classical Heegaard Floer homology, the definition of $\pi_{2}(\mathbf{x}, \mathbf{y})$ does not include this requirement.
} 
$\mathcal{M}^{B}(\mathbf{x}, \mathbf{y}, \vec{\rho})$ of $J$-holomorphic curves in $\Sigma \times[0,1] \times \mathbb{R}$ in the homology class $B$ whose asymptotics near $\partial \Sigma \times[0,1] \times \mathbb{R}$ are specified by $\vec{\rho}$. In particular, if $\operatorname{ind}(B, \vec{\rho})=1$, then this moduli space contains finitely many points. We do not give the full definition here; see [10, Chapter 5] for the details.

For each $\mathbf{x}, \mathbf{y} \in \mathfrak{S}(\mathbf{x})$ and $B \in \pi_{2}(\mathbf{x}, \mathbf{y})$, define

$$
a_{\mathbf{x}, \mathbf{y}}^{B}=\sum_{\substack{\left\{\vec{\rho}=\left(-\rho_{I_{1}}, \ldots,-\rho_{I_{k}}\right) \mid \\(B, \vec{\rho}) \text { compatible, } \\ \text { ind }(B, \vec{\rho})=1\right\}}} \#\left(\mathcal{M}^{B}(\mathbf{x}, \mathbf{y}, \vec{\rho})\right) \rho_{I_{1}} \ldots \rho_{I_{k}} \in \mathcal{A},
$$

where the count of points in $\mathcal{M}^{B}(\mathbf{x}, \mathbf{y}, \vec{\rho})$ is taken modulo 2 . We define $\delta_{1}: X(\mathcal{H}) \rightarrow$ $\mathcal{A} \underset{\mathcal{I}}{\otimes} X(\mathcal{H})$ by

$$
\delta_{1}(\mathbf{x})=\sum_{\mathbf{y} \in \mathfrak{S}(\mathcal{H})} \sum_{B \in \pi_{2}(\mathbf{x}, \mathbf{y})} a_{\mathbf{x}, \mathbf{y}}^{B} \otimes \mathbf{y}
$$

This defines a type $D$ structure, which we denote $\widehat{\mathrm{CFD}}(\mathcal{H})$. The verification of (2.2) is a version of the standard $\partial^{2}=0$ argument in Floer theory. (Henceforth, if $\mathbf{x}, \mathbf{y}$, and $\vec{\rho}$ are understood from the context, we shall write $\mathcal{M}(B)$ in place of $\mathcal{M}^{B}(\mathbf{x}, \mathbf{y}, \vec{\rho})$. If we need to be explicit about the choice of complex structure $J$ on $\Sigma$, we shall write $\mathcal{M}_{J}(B)$ or $\mathcal{M}_{J}^{B}(\mathbf{x}, \mathbf{y}, \vec{\rho})$.)

\section{Proposition 2.1.}

(1) The only sequences of chords that can contribute nonzero terms to $\delta_{1}$ are the empty sequence, $\left(-\rho_{1}\right),\left(-\rho_{2}\right),\left(-\rho_{3}\right),\left(-\rho_{1},-\rho_{2}\right),\left(-\rho_{2},-\rho_{3}\right),\left(-\rho_{123}\right)$, and $\left(-\rho_{1},-\rho_{2},-\rho_{3}\right)$. Therefore, only classes whose multiplicities in the boundary regions of $\Sigma$ are 0 or 1 can count for $\delta_{1}$.

(2) If $B \in \pi_{2}(\mathbf{x}, \mathbf{y})$ is a positive class whose domain has multiplicity 1 in the regions abutting $\rho_{1}$ and $\rho_{2}$ (resp. $\rho_{2}$ and $\rho_{3}$ ) and 0 in the region abutting $\rho_{3}$ (resp. $\rho_{1}$ ), then $B$ may count for the differential only if $\mathbf{x}$ and $\mathbf{y}$ contain points of $\alpha_{1}^{a}\left(\right.$ resp. $\left.\alpha_{2}^{a}\right)$.

Proof. For the first statement, the only other sequences of chords for which the product of algebra elements in the definition of $a_{\mathbf{x}, \mathbf{y}}^{B}$ is nonzero are $\left(-\rho_{12}\right),\left(-\rho_{23}\right)$, $\left(-\rho_{1},-\rho_{23}\right)$, and $\left(-\rho_{12},-\rho_{3}\right)$. The two latter sequences are not strongly boundary monotonic. If $B \in \pi_{2}(\mathbf{x}, \mathbf{y})$ is a positive class compatible with $\left(-\rho_{12}\right)$, then $\mathbf{x}$ and $\mathbf{y}$ both contain points on $\alpha_{1}^{a}$, since otherwise $B$ would have a boundary component without a $\beta$ segment. Therefore, $I_{D}(\mathbf{y})=\iota_{1}$. Since the tensor product is taken over the ring of idempotents,

$$
\rho_{12} \otimes \mathbf{y}=\rho_{12} \otimes \iota_{1} \mathbf{y}=\rho_{12} \iota_{1} \otimes \mathbf{y}=0,
$$

so the contribution of $B$ to $\delta_{1}(\mathbf{x})$ is zero. A similar argument applies for the sequence $\left(-\rho_{23}\right)$. The second statement follows immediately from the same argument.

The invariant $\widehat{\mathrm{CFA}}$ is a type $A$ structure associated to a bordered Heegaard diagram whose boundary is identified with $\mathcal{Z}$. We do not give all the details here. The 
analogue of Proposition 2.1 does not hold for $\widehat{\mathrm{CFA}}$; one must consider domains with arbitrary multiplicities on the boundary and a much larger family of sequences of chords. Therefore, it is generally easier to compute $\widehat{\mathrm{CFD}}$.

We conclude this section with the gluing theorem:

Theorem 2.2 (Lipshitz-Ozsváth-Thurston [10]). Suppose $\mathcal{Y}_{1}$ and $\mathcal{Y}_{2}$ are bordered 3-manifolds, and $Y=Y_{1} \cup_{\phi} Y_{2}$ is the manifold obtained by gluing them together along their boundaries, where $\phi:-\partial Y_{1} \rightarrow \partial Y_{2}$ is the map induced by the bordered structures. Then

$$
\widehat{\mathrm{CF}}(Y) \simeq \widehat{\mathrm{CFA}}\left(\mathcal{Y}_{1}\right) \otimes \widehat{\mathrm{CFD}}\left(\mathcal{Y}_{2}\right)
$$

provided that at least one of the modules is bounded (so that the box tensor product is defined).

2.4. Bimodules. In [11, Lipshitz, Ozsváth, and Thurston also define invariants for a bordered manifold with two boundary components. Essentially, this consists of a manifold $Y$ with two boundary components $\partial_{L} Y$ and $\partial_{R} Y$, with parametrizations of the two boundary components just like in the single-component case, and a framed arc connecting the two boundary components. Here, we assume that both boundary components are tori; see [11, Chapter 5] for the full definition.

If both boundary components are parametrized by $-F(\mathcal{Z})$, the associated invariant is a type $D D$ structure over two copies of $\mathcal{A}$, denoted $\widehat{\operatorname{CFDD}}(\mathcal{Y})$; if both are parametrized by $F(\mathcal{Z})$, the invariant is a type $A A$ structure, denoted $\widehat{\operatorname{CFAA}}(\mathcal{Y})$; and similarly there are invariants $\widehat{\mathrm{CFAD}}(\mathcal{Y})$ and $\widehat{\mathrm{CFDA}}(\mathcal{Y})$. For simplicity, we denote the two copies of $\mathcal{A}$ by $\mathcal{A}_{\rho}$ and $\mathcal{A}_{\sigma}$; in the latter, the Reeb elements are written $\sigma_{1}, \sigma_{2}$, etc.

In fact, we consider only a direct summand of each bimodule, denoted $\widehat{\operatorname{CFDD}}(\mathcal{Y}, 0)$, $\widehat{\mathrm{CFAA}}(\mathcal{Y}, 0)$, etc., which is all that is necessary to compute the Floer complex of a manifold obtained by gluing two separate one-boundary-component manifolds to the two boundary components of $Y$. The other summands are only necessary if one wishes to glue together the two boundary components of $Y$.

As in the previous discussion, we describe only the construction of $\widehat{\mathrm{CFDD}}$. A bordered manifold with two toroidal boundary components may be presented by an arced bordered Heegaard diagram

$$
\mathcal{H}=\left(\Sigma,\left\{\alpha_{1}^{c}, \ldots, \alpha_{g-2}^{c}, \alpha_{1}^{L}, \alpha_{2}^{L}, \alpha_{1}^{R}, \alpha_{2}^{R}\right\},\left\{\beta_{1}, \ldots, \beta_{g}\right\}, \mathbf{z}\right),
$$

where now $\partial \Sigma$ has two components $\partial_{L} \Sigma$ and $\partial_{R} \Sigma$, on which the $\operatorname{arcs} \alpha_{i}^{L}$ and $\alpha_{i}^{R}$ have their respective boundaries, and $\mathbf{z}$ is an arc in the complement of all the $\alpha$ and $\beta$ circles and $\alpha$ arcs connecting the two boundary components.

We define $\mathfrak{S}(\mathcal{H})$ and $X(\mathcal{H})$ just in the single-boundary-component case. Let $\mathfrak{S}(\mathcal{H}, 0)$ be the subset of $\mathfrak{S}(\mathcal{H})$ consisting of $g$-tuples $\mathbf{x}$ containing one point in $\alpha_{1}^{L} \cup \alpha_{2}^{L}$ and one point in $\alpha_{1}^{R} \cup \alpha_{2}^{R}$, and let $X(\mathcal{H}, 0)$ be the $\mathbb{F}$-vector space generated by $\mathfrak{S}(\mathcal{H}, 0)$. This is the underlying vector space for the invariants $\widehat{\operatorname{CFDD}}(\mathcal{H}, 0), \widehat{\operatorname{CFAA}}(\mathcal{H}, 0)$, etc. 
To define $\widehat{\mathrm{CFDD}}(\mathcal{H}, 0)$, identify both boundary components of $\Sigma$ with $-\mathcal{Z}$. Each generator of $\widehat{\operatorname{CFDD}}(\mathcal{H}, 0)$ has associated idempotents in $\mathcal{A}_{\rho}$ and $\mathcal{A}_{\sigma}$, as in (2.4). The differential

$$
\delta_{1}: X(\mathcal{H}, 0) \rightarrow\left(\mathcal{A}_{\rho} \otimes \mathcal{A}_{\sigma}\right) \underset{\mathcal{I}_{\rho} \otimes \mathcal{I}_{\sigma}}{\otimes} X(\mathcal{H}, 0)
$$

is then defined essentially the same way as with $\widehat{\mathrm{CFD}}$ of a single-boundary-component diagram. Specifically, for a homology class $B \in \pi_{2}(\mathbf{x}, \mathbf{y})$ and sequences of chords $\vec{\rho}=\left(-\rho_{I_{1}}, \ldots,-\rho_{I_{k}}\right)$ and $\vec{\sigma}=\left(-\sigma_{J_{1}}, \ldots,-\sigma_{J_{l}}\right)$ on the two boundary components, the definitions of compatibility and of the index $\operatorname{ind}(B, \vec{\rho}, \vec{\sigma})$ are as above. Define

$$
a_{\mathbf{x}, \mathbf{y}}^{B}=\sum_{\substack{\{(\vec{\rho}, \vec{\sigma}) \mid \\(B, \vec{\rho}, \vec{\sigma}) \text { compatible, } \\ \text { ind }(B, \vec{\rho}, \vec{\sigma})=1\}}} \#\left(\mathcal{M}^{B}(\mathbf{x}, \mathbf{y}, \vec{\rho}, \vec{\sigma})\right) \rho_{I_{1}} \ldots \rho_{I_{k}} \otimes \sigma_{J_{1}} \ldots \sigma_{J_{l}} \in \mathcal{A}_{\rho} \otimes \mathcal{A}_{\sigma} .
$$

The map $\delta_{1}$ is then given by (2.6) just as above. An analogue of Proposition 2.1 also holds in this setting. For further details, see [11, Section 6].

The gluing theorem generalizes naturally to bimodules. For instance, if $Y_{1}$ has a single boundary component parametrized by $F(\mathcal{Z}), Y_{2}$ has two boundary components parametrized by $-F(\mathcal{Z})$, and $\phi:-\partial Y_{1} \rightarrow \partial_{L} Y_{2}$ is the map induced by the parametrizations, then

$$
\widehat{\mathrm{CFD}}\left(\mathcal{Y}_{1} \cup_{\phi} \mathcal{Y}_{2}\right) \simeq \widehat{\mathrm{CFA}}\left(\mathcal{Y}_{1}\right) \underset{\mathcal{A}_{\rho}}{\otimes} \widehat{\mathrm{CFDD}}\left(\mathcal{Y}_{2}, 0\right)
$$

The remaining generalizations are found in [11, Theorems 11, 12].

Finally, we mention the identity AA bimodule [11, Subsection 10.1]. Consider the manifold $\mathbb{I}=F(\mathcal{Z}) \times I$. Parametrize $\partial_{R} Y=F(\mathcal{Z}) \times\{1\}$ by inclusion and $\partial_{L} Y=F(\mathcal{Z}) \times\{0\}$ (whose boundary-induced orientation is opposite to the standard orientation of $F(\mathcal{Z})$ ) by the composition $F(\mathcal{Z}) \stackrel{r}{\rightarrow}-F(\mathcal{Z}) \hookrightarrow F(\mathcal{Z}) \times\{0\}$; thus, both boundary components are parametrized by $F(\mathcal{Z})$ as opposed to $-F(\mathcal{Z})$. The bijection between bordered manifolds with boundary $-F(\mathcal{Z})$ and bordered manifolds with boundary $F(\mathcal{Z})$ may be given by $Y \mapsto Y \cup \mathbb{I}$. Thus, if $\mathcal{H}$ is any bordered Heegaard diagram with one boundary component, then the type $A$ module $\widehat{\mathrm{CFA}}(\mathcal{H})$ (where we identify $\partial \Sigma$ with $\mathcal{Z}$ ) is chain homotopy equivalent to $\widehat{\mathrm{CFAA}}(\mathbb{I}, 0) \otimes \widehat{\mathrm{CFD}}(\mathcal{H})$ (where, in the second factor, we identify $\partial \Sigma$ with $-\mathcal{Z}$ ) 2 As mentioned above, it is easier to compute $\widehat{\mathrm{CFD}}$ explicitly from a Heegaard diagram than $\widehat{\mathrm{CFA}}$; by taking a tensor product with $\widehat{\mathrm{CFAA}}(\mathbb{I}, 0)$, we can always avoid the latter.

Theorem 2.3 (Lipshitz-Ozsváth-Thurston). The type AA module $\widehat{\mathrm{CFAA}}(\mathbb{I}, 0)$ has generators $w_{1}, w_{2}, x, y, z_{1}, z_{2}$, with $\mathcal{A}_{\infty}$ multiplications as illustrated in Figure 4 . That

\footnotetext{
${ }^{2}$ Our presentation here is a bit different from that of Lipshitz, Ozsváth, and Thurston, who describe $\widehat{\mathrm{CFAA}}(\mathbb{I}, 0)$ as a bimodule over two separate algebras, $\mathcal{A}(\mathcal{Z})$ and $\mathcal{A}(-\mathcal{Z})=\mathcal{A}(\mathcal{Z})^{\mathrm{op}}$. The latter happens to be isomorphic to $\mathcal{A}(\mathcal{Z})$ because of the involution $r$, so the two boundary components of $\mathcal{I}$ are effectively interchangeable. For the purposes of this introduction, we find it clearer to suppress the distinction between $\mathcal{A}(\mathcal{Z})$ and $\mathcal{A}(-\mathcal{Z})$, at the cost of being more explicit about $r$.
} 


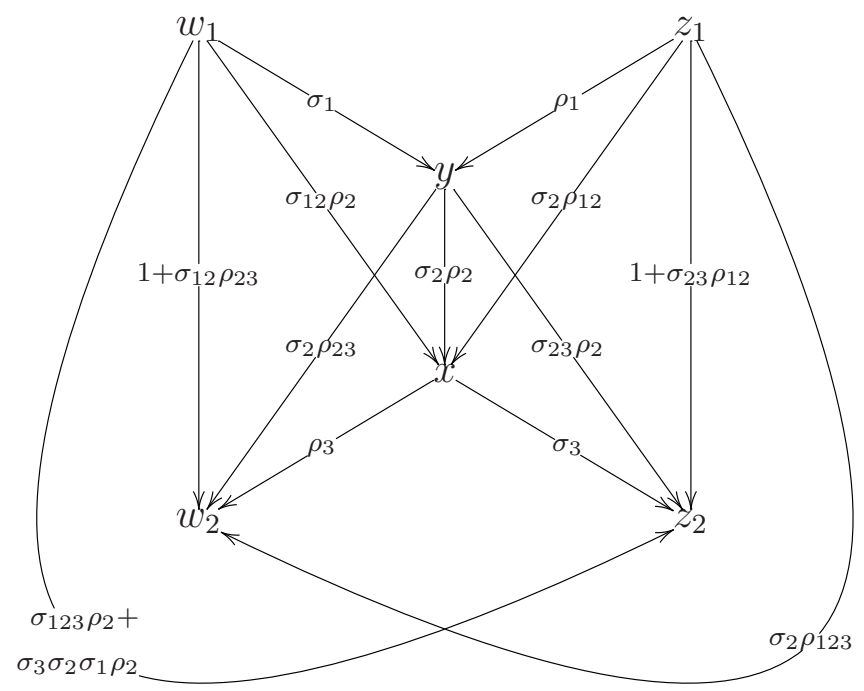

Figure 4. The identity $A A$ bimodule, $\widehat{\mathrm{CFAA}}(\mathcal{I}, 0)$.

is, $m_{1,0,0}\left(w_{1}\right)=w_{2}, m_{1,0,1}\left(w_{1}, \sigma_{1}\right)=y, m_{1,1,1}\left(w_{1}, \sigma_{12}, \rho_{23}\right)=w_{2}$, and so on. (See below for more on this notation.)

2.5. Knots in bordered manifolds. A doubly-pointed bordered Heegaard diagram consists of a bordered Heegaard diagram $\mathcal{H}=(\Sigma, \boldsymbol{\alpha}, \boldsymbol{\beta}, z)$ along with an additional basepoint $w \in \Sigma \backslash(\boldsymbol{\alpha} \cup \boldsymbol{\beta})$. As explained in [10, Section 11.4], a doubly-pointed diagram determines a knot $K \subset Y$ with a single point of $K$ meeting the basepoint on $\partial Y$; the isotopy class of $K$ relative to this point is invariant under Heegaard moves missing $w$. Lipshitz, Ozsváth, and Thurston define invariants $\operatorname{CFD}^{-}(Y, K)$ and $\mathrm{CFA}^{-}(Y, K)$ by working over the algebra $\mathcal{A} \otimes \mathbb{F}[U]$, where the $U$ powers record the multiplicity of $w$ in each domain that counts for the differential or multiplications.

If the knot $K$ is nulhomologous in $Y$, we prefer the following alternate perspective. Push $K$ slightly into the interior of $Y$ (so that it now misses the boundary), and let $F$ be a Seifert surface for $K$. Just as in ordinary knot Floer homology [15, 18, each generator $\mathbf{x} \in \mathfrak{S}(\mathcal{H})$ has an associated relative $\operatorname{spin}^{c} \operatorname{structure} \mathfrak{s}_{z, w}(\mathbf{x}) \in \operatorname{Spin}^{c}(Y, K)$, and we may define an Alexander grading on $\mathfrak{S}(\mathcal{H})$ by

$$
A(x)=\frac{1}{2}\left\langle c_{1}\left(\mathfrak{s}_{z, w}(\mathbf{x})\right),[F]\right\rangle,
$$

where $c_{1}\left(\mathfrak{s}_{z, w}(\mathbf{x})\right) \in H^{2}(Y, K)$ and $[F] \in H_{2}(Y, K)$. The grading difference between two generators is given by

$$
A(x)-A(y)=n_{w}(B)
$$

where $B \in \pi_{2}(\mathbf{x}, \mathbf{y})$ is any domain from $\mathbf{x}$ to $\mathbf{y}$. To verify that the right-hand side of (2.8) is well-defined, note that for any periodic class $P \in \pi_{2}(\mathbf{x}, \mathbf{x}), n_{w}(P)$ equals the intersection number of $K$ with the homology class in $H_{2}(Y, \partial Y)$ corresponding 
to $P$, which must be zero since $K$ is nulhomologous. Further details are completely analogous to [15, 18].

The Alexander grading on $X(\mathcal{H})$ determines a filtration on $\widehat{\mathrm{CFA}}(\mathcal{H})$ or $\widehat{\mathrm{CFD}}(\mathcal{H})$, since any domain that counts for the differential or $\mathcal{A}_{\infty}$ multiplications has nonnegative multiplicity at $w$. We denote the filtered chain homotopy type by $\widehat{\mathrm{CFA}}(\mathcal{Y}, K)$ or $\widehat{\mathrm{CFD}}(\mathcal{Y}, K)$.

When we evaluate a tensor product $\widehat{\mathrm{CFA}}\left(\mathcal{Y}_{1}\right) \otimes \widehat{\mathrm{CFD}}\left(\mathcal{Y}_{2}\right)$, a knot filtration on one factor extends naturally to a filtration on the whole complex, which agrees with the filtration that the knot induces on $\widehat{\mathrm{CF}}\left(Y_{1} \cup Y_{2}\right)$.

A nulhomologous knot in a bordered manifold with two boundary components may be handled similarly. For invariance, one point of the knot must be constrained to lie on the arc connecting the two boundary components, and isotopies must be fixed in a neighborhood of that point.

2.6. The edge reduction algorithm. We now describe the well-known "edge reduction" procedure for chain complexes and its extension to $\mathcal{A}_{\infty}$ modules.

Suppose $(C, \partial)$ is a free chain complex with basis $\left\{x_{1}, \ldots, x_{n}\right\}$ over a ring $R$. For each $i, j$, let $a_{i j}$ be the coefficient of $x_{j}$ in $\partial x_{i}$ with respect to this basis. If some $a_{i j}$ is invertible in $R$, define a new basis $\left\{y_{1}, \ldots, y_{n}\right\}$ by setting $y_{i}=x_{i}, y_{j}=\partial x_{i}$, and for each $k \neq i, j, y_{k}=x_{k}-a_{k j} a_{i j}^{-1} x_{i}$, where $a_{k j}$ is the coefficient of $x_{j}$ in $\partial x_{k}$. With respect to the new basis, the coefficient of $y_{j}$ in $\partial y_{k}$ is zero, so the subspace spanned by $y_{i}$ and $y_{j}$ is a direct summand subcomplex with trivial homology. Thus, $C$ is chain homotopy equivalent to the subcomplex $C^{\prime}$ spanned by $\left\{y_{k} \mid k \neq i, j\right\}$, in which the coefficient of $y_{l}$ in $\partial y_{k}$ is $a_{k l}-a_{k j} a_{i j}^{-1} a_{i l}$.

When $R=\mathbb{F}_{2}$, a convenient way to represent a chain complex $(C, \partial)$ with basis $\left\{x_{i}\right\}$ is a directed graph $\Gamma_{C, \partial,\left\{x_{i}\right\}}$ with vertices corresponding to basis elements and an edge from $x_{i}$ to $x_{j}$ whenever $a_{i j}=1$. To obtain $\Gamma_{C^{\prime}, \partial,\left\{y_{k}\right\}}$ from $\Gamma_{C, \partial,\left\{x_{i}\right\}}$ as above, we delete the vertices $x_{i}$ and $x_{j}$ and any edges going into or out of them. For each $k$ and $l$ with edges $x_{k} \rightarrow x_{j}$ and $x_{i} \rightarrow x_{l}$, we either add an edge from $x_{k}$ to $x_{l}$ (if there was not one previously) or eliminate the edge from $x_{k}$ to $x_{l}$ (if there was one). We call this procedure canceling the edge from $x_{i}$ to $x_{j}$. The vertices of the resulting graph should be labeled with $\left\{y_{k} \mid k \neq i, j\right\}$, but by abuse of notation we frequently continue to refer to them with $\left\{x_{k} \mid k \neq i, j\right\}$ instead.

By iterating this procedure until no more edges remain, we compute the homology of $C$. If the matrix $\left(a_{i j}\right)$ is sparse, this tends to be a very efficient algorithm for computing homology. If $C$ is a graded complex and the basis $\left\{x_{1}, \ldots, x_{n}\right\}$ consists of homogeneous elements, then $y_{k}$ is clearly homogeneous with the same grading as $x_{k}$, so we can compute the homology as a graded group.

If $C$ has a filtration $\cdots \subseteq F_{p} \subseteq F_{p+1} \subseteq \cdots$, the filtration level of an element of $C$ is the unique $p$ for which that element is in $F_{p} \backslash F_{p-1}$. To compute the spectral sequence associated to the filtration, we cancel edges in increasing order of the amount by which they decrease filtration level. At each stage, this guarantees that the filtration level of $y_{k}$ equals that of $x_{k}$. The complex that remains after we delete all edges 
that decrease filtration level by $k$ is the $E^{k+1}$ page in the spectral sequence, and the vertices that remain after all edges are deleted is the $E^{\infty}$ page. In particular, when $C=\widehat{\mathrm{CF}}\left(S^{3}, K\right)$, the filtered complex associated to a knot $K \subset S^{3}$, the total homology of $C$ is $\widehat{\operatorname{HF}}\left(S^{3} ; \mathbb{F}\right) \cong \mathbb{F}$, so a unique vertex survives after all cancellations are complete. The filtration level of this vertex is, by definition, the invariant $\tau(K)$.

More generally, over an arbitrary ring $R$, we may represent $(C, \partial)$ by a labeled, directed graph, where now we label an edge from $x_{i}$ to $x_{j}$ by $a_{i j}$, often omitting the label when $a_{i j}=1$. When we cancel an unlabeled edge from $x_{i}$ to $x_{j}$, we replace a zigzag

$$
x_{k} \stackrel{a_{k j}}{\longrightarrow} x_{j} \longleftarrow x_{i} \stackrel{a_{i l}}{\longrightarrow} x_{l}
$$

with an edge

$$
x_{k} \stackrel{-a_{k l} a_{i l}}{\longrightarrow} x_{l}
$$

if no such edge existed previously, and either relabel or delete such an edge if it did exist. Of course, when $R$ is not a field, this procedure is not guaranteed to eliminate all edges or to yield a result that is independent of the choice of the order in which the edges are deleted, but it is still often a useful way to simplify a chain complex.

The same procedure works for type $D$ structures over the torus algebra $\mathcal{A}$, as can be seen by looking at the ordinary differential module obtained by taking the tensor product with $\mathcal{A}$ as above.

Edge cancellation for type $A$ structures is slightly more complicated. We work only with bounded modules for simplicity. Suppose $M$ is a bounded type $A$ structure over $\mathcal{A}$ with a basis $\left\{x_{1}, \ldots, x_{n}\right\}$. As above, we may describe the multiplications using a matrix of formal sums of finite sequences of elements of $\mathcal{A}$, and we may represent the nonzero entries using a labeled graph. The empty sequence will be denoted by 1 , and we often omit the label of an edge whose label is 1 . If there is an unlabeled edge from $x_{i}$ to $x_{j}$ then we may cancel $x_{i}$ and $x_{j}$, replacing a zigzag

$$
x_{k} \stackrel{\left(a_{1}, \ldots, a_{p}\right)}{\longrightarrow} x_{j} \longleftarrow x_{i} \stackrel{\left(b_{1}, \ldots, b_{q}\right)}{\longrightarrow} x_{l}
$$

by an edge

$$
x_{k} \stackrel{\left(a_{1}, \ldots, a_{p}, b_{1}, \ldots, b_{q}\right)}{\longrightarrow} x_{l}
$$

(or eliminating such an edge if one already exists). The $\mathcal{A}_{\infty}$ module $M^{\prime}$ described by the resulting graph is then $\mathcal{A}_{\infty}$ chain homotopic to $M$. If $M$ is a filtered $\mathcal{A}_{\infty}$-module and the edge being canceled is filtration-preserving (i.e., $x_{i}$ and $x_{j}$ have the same filtration level), then $M^{\prime}$ is filtered $\mathcal{A}_{\infty}$ chain homotopic to $M$. Similar techniques may also be used for bimodules. (As in Figure 4, we frequently omit the parentheses and commas on the edge labels for conciseness; with this notation, concatenation does not indicate multiplication in $\mathcal{A}$.)

2.7. $\widehat{\mathrm{CFD}}$ of knot complements. For any knot $K$, let $X_{K}$ denote the exterior of $K$. For $t \in \mathbb{Z}$, let $\mathcal{X}_{K}^{t}$ denote the bordered structure on $X_{K}$ determined by a map $\phi:-F(\mathcal{Z}) \rightarrow \partial X_{K}$ sending $h_{1}$ to a $t$-framed longitude (relative to the Seifert framing) and $h_{2}$ to a meridian of $K$. Lipshitz, Ozsváth, and Thurston [10] give a complete 
computation of $\widehat{\mathrm{CFD}}\left(\mathcal{X}_{K}^{t}\right)$ in terms of the knot Floer complex of $K$, which we now describe.

In the computation that follows, we will need to work with two different framed knot complements, $\mathcal{X}_{J}^{s}$ and $\mathcal{X}_{K}^{t}$. We first state the results for $\widehat{\operatorname{CFD}}\left(\mathcal{X}_{J}^{s}\right)$ and then indicate how to modify the notation for $\widehat{\mathrm{CFD}}\left(\mathcal{X}_{K}^{t}\right)$. Define $r=|2 \tau(J)-s|$, and say that $\operatorname{dim} \widehat{\operatorname{HFK}}\left(S^{3}, J\right)=2 n+1$.

We may find two distinguished bases for $\mathrm{CFK}^{-}\left(S^{3}, J\right)$ : a "vertically reduced" basis $\left\{\tilde{\xi}_{0}, \ldots, \tilde{\xi}_{2 n}\right\}$, with "vertical arrows" $\tilde{\xi}_{2 j-1} \rightarrow \tilde{\xi}_{2 j}$ of length $k_{j} \in \mathbb{N}$, and a "horizontally reduced" basis $\left\{\tilde{\eta}_{0}, \ldots, \tilde{\eta}_{2 n}\right\}$, with "horizontal arrows" $\tilde{\xi}_{2 j-1} \rightarrow \tilde{\xi}_{2 j}$ of length $l_{j} \in \mathbb{N}$. (See [10, Section 11.5] for the definitions.) Denote the change-of-basis matrices by $\left(x_{p, q}\right)$ and $\left(y_{p, q}\right)$, so that

$$
\tilde{\xi}_{p}=\sum_{q=0}^{2 n} x_{p, q} \tilde{\eta}_{q} \quad \text { and } \quad \tilde{\eta}_{p}=\sum_{q=0}^{2 n} y_{p, q} \tilde{\xi}_{q} .
$$

In all known instances, the two bases may be taken to be equal as sets (up to a permutation), but it has not been proven that this holds in general.

According to [10, Theorems 11.27, A.11], the structure of $\widehat{\mathrm{CFD}}\left(\mathcal{X}_{J}^{t}\right)$ is as follows. The part in idempotent $\iota_{0}$ (i.e., $\iota_{0} \widehat{\mathrm{CFD}}\left(\mathcal{X}_{J}^{s}\right)$ ) has dimension $2 n+1$, with designated bases $\left\{\xi_{0}, \ldots, \xi_{2 n}\right\}$ and $\left\{\eta_{0}, \ldots, \eta_{2 n}\right\}$ related by (2.9) without the tildes. The part in idempotent $\iota_{1}$ (i.e., $\left.\iota_{1} \widehat{\operatorname{CFD}}\left(\mathcal{X}_{J}^{s}\right)\right)$ has dimension $r+\sum_{j=1}^{n}\left(k_{j}+l_{j}\right)$, with basis

$$
\left\{\gamma_{1}, \ldots, \gamma_{r}\right\} \cup \bigcup_{j=1}^{n}\left\{\kappa_{1}^{j}, \ldots, \kappa_{k_{j}}^{j}\right\} \cup \bigcup_{j=1}^{n}\left\{\lambda_{1}^{j}, \ldots, \lambda_{l_{j}}^{j}\right\} .
$$

For $j=1, \ldots, n$, corresponding to the vertical arrow $\tilde{\eta}_{2 j-1} \rightarrow \tilde{\eta}_{2 j}$, there are differentials

$$
\xi_{2 j} \stackrel{\rho_{123}}{\longrightarrow} \kappa_{1}^{j} \stackrel{\rho_{23}}{\longrightarrow} \cdots \stackrel{\rho_{23}}{\longrightarrow} \kappa_{k_{j}}^{j} \stackrel{\rho_{1}}{\longleftarrow} \xi_{2 j-1} .
$$

(In other words, $\delta_{1}\left(\xi_{2 j}\right)$ has a $\rho_{123} \otimes \kappa_{1}^{j}$ term, and so on.) We refer to the subspace of $\widehat{\mathrm{CFD}}\left(\mathcal{X}_{J}^{s}\right)$ spanned by the generators in $(2.10)$ as a vertical stable chain. Similarly, corresponding to the horizontal arrow $\eta_{2 j-1} \rightarrow \eta_{2 j}$ of length $l_{j}$, there are differentials

$$
\eta_{2 j-1} \stackrel{\rho_{3}}{\longrightarrow} \lambda_{1}^{j} \stackrel{\rho_{23}}{\longrightarrow} \cdots \stackrel{\rho_{23}}{\longrightarrow} \lambda_{l_{j}}^{j} \stackrel{\rho_{2}}{\longrightarrow} \eta_{2 j}
$$

and the generators here span a horizontal stable chain. Finally, the generators $\xi_{0}, \eta_{0}, \gamma_{1}, \ldots, \gamma_{r}$ span the unstable chain, with differentials depending on $s$ and $\tau(J)$ :

$$
\begin{cases}\eta_{0} \stackrel{\rho_{3}}{\longrightarrow} \gamma_{1} \stackrel{\rho_{23}}{\longrightarrow} \cdots \stackrel{\rho_{23}}{\longrightarrow} \gamma_{r} \stackrel{\rho_{1}}{\longleftarrow} \xi_{0} & s<2 \tau(J) \\ \xi_{0} \stackrel{\rho_{12}}{\longrightarrow} \eta_{0} & s=2 \tau(J) \\ \xi_{0} \stackrel{\rho_{123}}{\longrightarrow} \gamma_{1} \stackrel{\rho_{23}}{\longrightarrow} \cdots \stackrel{\rho_{23}}{\longrightarrow} \gamma_{r} \stackrel{\rho_{2}}{\longrightarrow} \eta_{0} & s>2 \tau(J) .\end{cases}
$$

In some instances, as with the unknot and the figure-eight knot, we may have $\xi_{0}=\eta_{0}$.

For $\widehat{\mathrm{CFD}}\left(\mathcal{X}_{K}^{t}\right)$, we modify the preceding two paragraphs by replacing all lowercase letters with capital letters. Specifically, $\iota_{0} \widehat{\mathrm{CFD}}\left(\mathcal{X}_{K}^{t}\right)$ has bases $\left\{\Xi_{0}, \ldots, \Xi_{2 N}\right\}$ 
and $\left\{\mathrm{H}_{0}, \ldots, \mathrm{H}_{2 N}\right\}$ related by change-of-basis matrices $\left(X_{P, Q}\right)$ and $\left(Y_{P, Q}\right)$ as in (2.9); $\iota_{1} \widehat{\mathrm{CFD}}\left(\mathcal{X}_{K}^{t}\right)$ has basis

$$
\left\{\Gamma_{1}, \ldots, \Gamma_{R}\right\} \cup \bigcup_{J=1}^{N}\left\{\mathrm{~K}_{1}^{J}, \ldots, \mathrm{K}_{K_{J}}^{J}\right\} \cup \bigcup_{J=1}^{N}\left\{\Lambda_{1}^{J}, \ldots, \Lambda_{L_{J}}^{J}\right\} ;
$$

and the differentials are just as in (2.10), (2.11), and (2.12), suitably modified 3 In the discussion below, we shall treat $\widehat{\mathrm{CFD}}\left(\mathcal{X}_{K}^{t}\right)$ as a type $D$ structure over a copy of $\mathcal{A}_{\sigma}$ in which the elements are referred to as $\sigma_{1}, \sigma_{2}$, etc., to facilitate taking the double tensor product.

In Section 4, we shall frequently use the following proposition to simplify computations:

Proposition 2.4. In the matrix entries for the higher maps $\delta_{k}$ for $\widehat{\mathrm{CFD}}\left(\mathcal{X}_{J}^{s}\right)$, there are no sequences of elements containing $\rho_{1} \otimes \rho_{2}, \rho_{1} \otimes \rho_{23}, \rho_{2} \otimes \rho_{3}$, or $\rho_{12} \otimes \rho_{3}$.

Proof. The only instances of $\rho_{1}$ in $\widehat{\mathrm{CFK}}\left(\mathcal{X}_{J}^{s}\right)$ are $\xi_{2 j-1} \stackrel{\rho_{1}}{\rightarrow} \kappa_{k_{j}}^{j}$ in the vertical chains and $\xi_{0} \stackrel{\rho_{1}}{\rightarrow} \gamma_{r}$ in the unstable chain when $s<2 \tau(J)$, and $\delta_{1}\left(\kappa_{k_{j}}^{j}\right)=\delta_{1}\left(\gamma_{r}\right)=0$. Thus, $\rho_{1} \otimes \rho_{2}$ and $\rho_{1} \otimes \rho_{23}$ may not occur in $\delta_{k}$. Similarly, the only instances of $\rho_{2}$ and $\rho_{12}$ are $\lambda_{l_{j}}^{j} \stackrel{\rho_{2}}{\rightarrow} \eta_{2 j}$ in the horizontal chains, $\gamma_{r} \stackrel{\rho_{2}}{\rightarrow} \eta_{0}$ in the unstable chain when $s>2 \tau(J)$, and $\xi_{0} \stackrel{\rho_{12}}{\longrightarrow} \eta_{0}$ when $s=2 \tau(J)$, and the only instances of $\rho_{3}$ are $\eta_{2 j-1} \stackrel{\rho_{3}}{\longrightarrow} \lambda_{1}^{j}$ in the horizontal chains and $\eta_{0} \stackrel{\rho_{3}}{\rightarrow} \gamma_{1}$ in the unstable chain when $s<2 \tau(J)$. Thus, no element that is at the head of a $\rho_{2}$ or $\rho_{12}$ arrow is also at the tail of a $\rho_{3}$ arrow.

\section{Direct computation of $\widehat{\mathrm{CFAA}}\left(Y, B_{3}\right)$}

As above, let $B=B_{1} \cup B_{2} \cup B_{3} \subset S^{3}$ denote the Borromean rings. Let $Y$ denote the complement of a neighborhood of $B_{1} \cup B_{2}$; then $B_{3}$ is a nulhomologous knot in $Y$. Let $\partial_{L} Y$ and $\partial_{R} Y$ be the boundary components coming from $B_{1}$ and $B_{2}$, respectively. We define a strongly bordered structure $\mathcal{Y}$ on $Y$ (in the sense of [11, Definition 5.1]) so that the map $\phi_{L}: F(\mathcal{Z}) \rightarrow \partial_{L} Y$ (resp. $\phi_{R}: F(\mathcal{Z}) \rightarrow \partial_{R} Y$ ) takes $h_{1}$ to a meridian of $B_{1}$ (resp. $B_{2}$ ) and $h_{2}$ to a Seifert-framed longitude of $B_{1}$ (resp. $B_{2}$ ). It follows that the glued manifold $\left(\mathcal{Y} \cup_{\partial_{L} Y} \mathcal{X}_{J}^{s}\right) \cup_{\partial_{R} Y} \mathcal{X}_{K}^{t}$, is $S^{3}$, and the image of $B_{3}$ is the knot $D_{J, s}(K, t)$ Thus, we must compute the filtered type $A A$ bimodule $\widehat{\operatorname{CFAA}}\left(\mathcal{Y}, B_{3}, 0\right)$.

\subsection{A Heegaard diagram for $\left(\mathcal{Y}, B_{3}\right)$.}

Proposition 3.1. The arced Heegaard diagram $\mathcal{H}$ (with extra basepoint $w$ ) shown in Figure 5 determines the pair $\left(\mathcal{Y}, B_{3}\right)$.

\footnotetext{
${ }^{3}$ The reader should take care to distinguish capital eta $(\mathrm{H})$ and kappa $(\mathrm{K})$ from the Roman letters $H$ and $K$. We find that the mnemonic advantage of using parallel notation for the generators of $\widehat{\mathrm{CFD}}\left(\mathcal{X}_{J}^{s}\right)$ and $\widehat{\mathrm{CFD}}\left(\mathcal{X}_{K}^{t}\right)$ outweighs any confusion that may arise.

${ }^{4}$ Because we are gluing the two boundary components of $\mathcal{Y}$ to separate single-boundary-component bordered manifolds, the choice of framed arc connecting $\partial_{L} Y$ and $\partial_{R} Y$ does not affect the final computation of the tensor product, so we suppress all reference to it.
} 


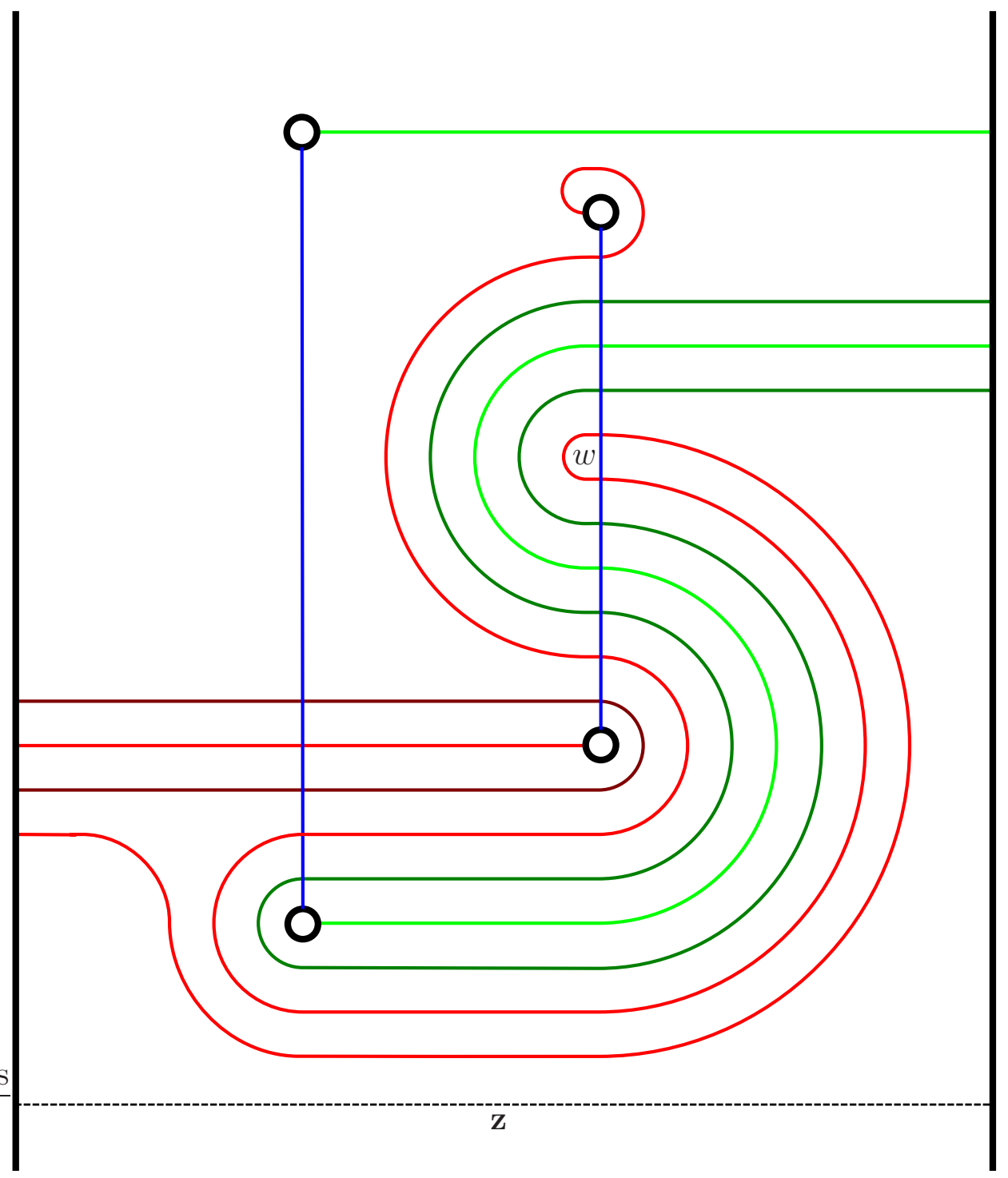

FigurE 5. The arced Heegaard diagram $\mathcal{H}$.

Proof. As in [11, Construction 5.4], by cutting along the arc $\mathbf{z}$, we obtain a bordered Heegaard diagram with a single boundary component, $\mathcal{H}_{d r}$, which we view as rectangle with two tunnels attached. After attaching 2-handles to $\mathcal{H}_{d r} \times[0,1]$ along $\boldsymbol{\beta} \times\{1\}$ and attaching a single 3 -handle, we may view the resulting manifold $Y\left(\mathcal{H}_{d r}\right)$ as $[-1,1] \times$ $\mathbb{R} \times[0, \infty) \subset \mathbb{R}^{3}$ plus a point at infinity, minus two tunnels as shown in Figure 6 . The boundary of $Y\left(\mathcal{H}_{d r}\right)$ is the union of two embedded copies of $F^{\circ}(\mathcal{Z})$ that are determined by the $\alpha$ arcs on each side; they intersect along a circle $A$. The extra basepoint $w$ determines a knot $C$ in $Y\left(\mathcal{H}_{d r}\right)$ with a single point on the boundary: the union of an arc connecting $w$ to $z$ in the complement of the $\alpha$ arcs and an arc 


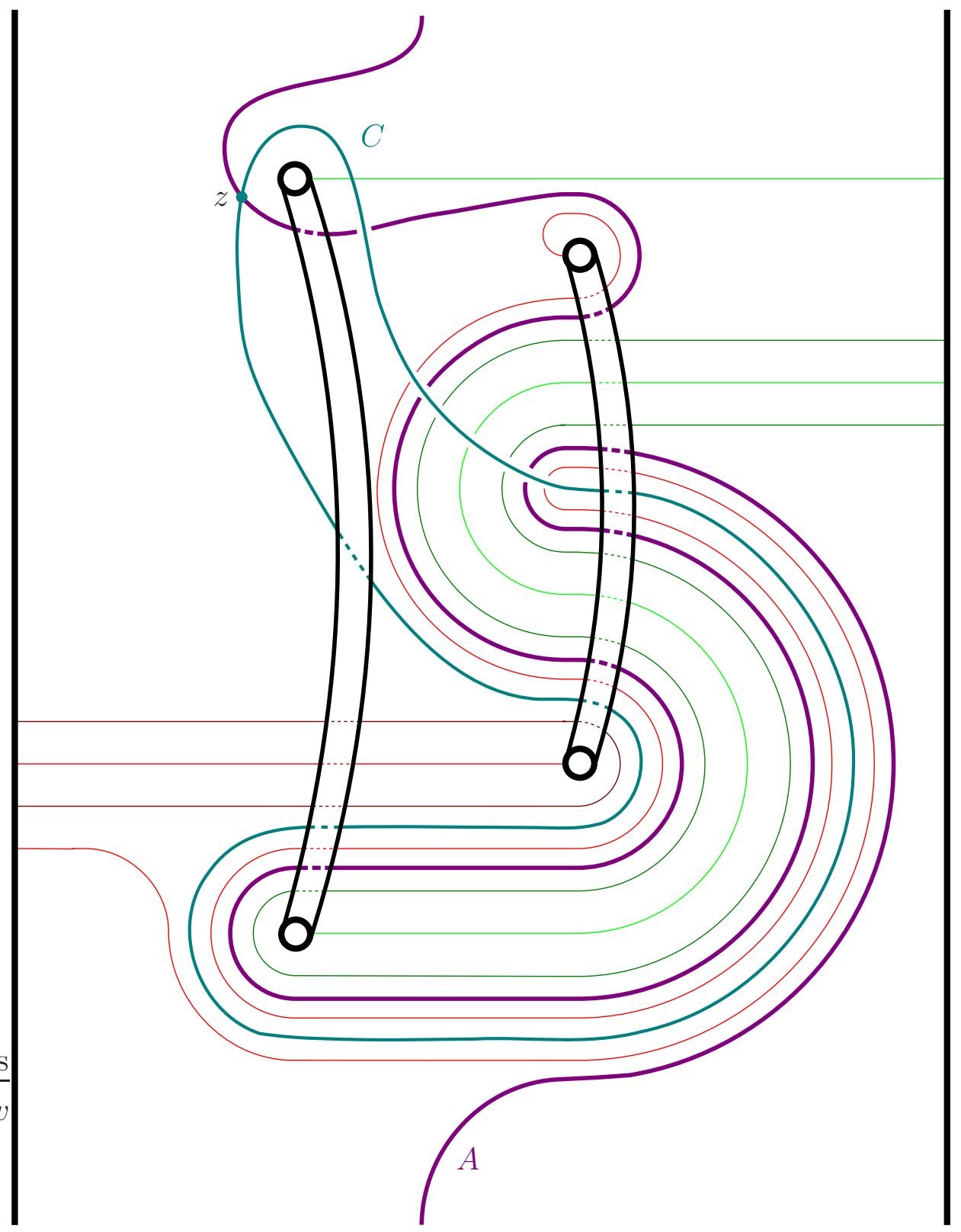

Figure 6 . The manifold $Y\left(\mathcal{H}_{d r}\right)$. The $\alpha$ arcs from $\mathcal{H}$ (the thin red and green curves) and the circle $A$ (purple) sit in the plane of the page, while the knot $C$ (turquoise) is above the plane of the page (i.e., in the interior of $Y\left(\mathcal{H}_{d r}\right)$ ) except at the point $z$.

connecting $z$ to $w$ in the complement of the $\beta$ circles, pushed into the interior of $Y\left(\mathcal{H}_{d r}\right)$ except at $z$. The curves $A$ and $C$ are both shown in Figure 6,

We obtain Figure 7 from Figure 6 by an isotopy that slides the tunnel on the right underneath the tunnel on the left. The circle $A$ can then be identified with the $y$-axis plus the point at infinity. To obtain $Y(\mathcal{H})$, we attach a three-dimensional two-handle 


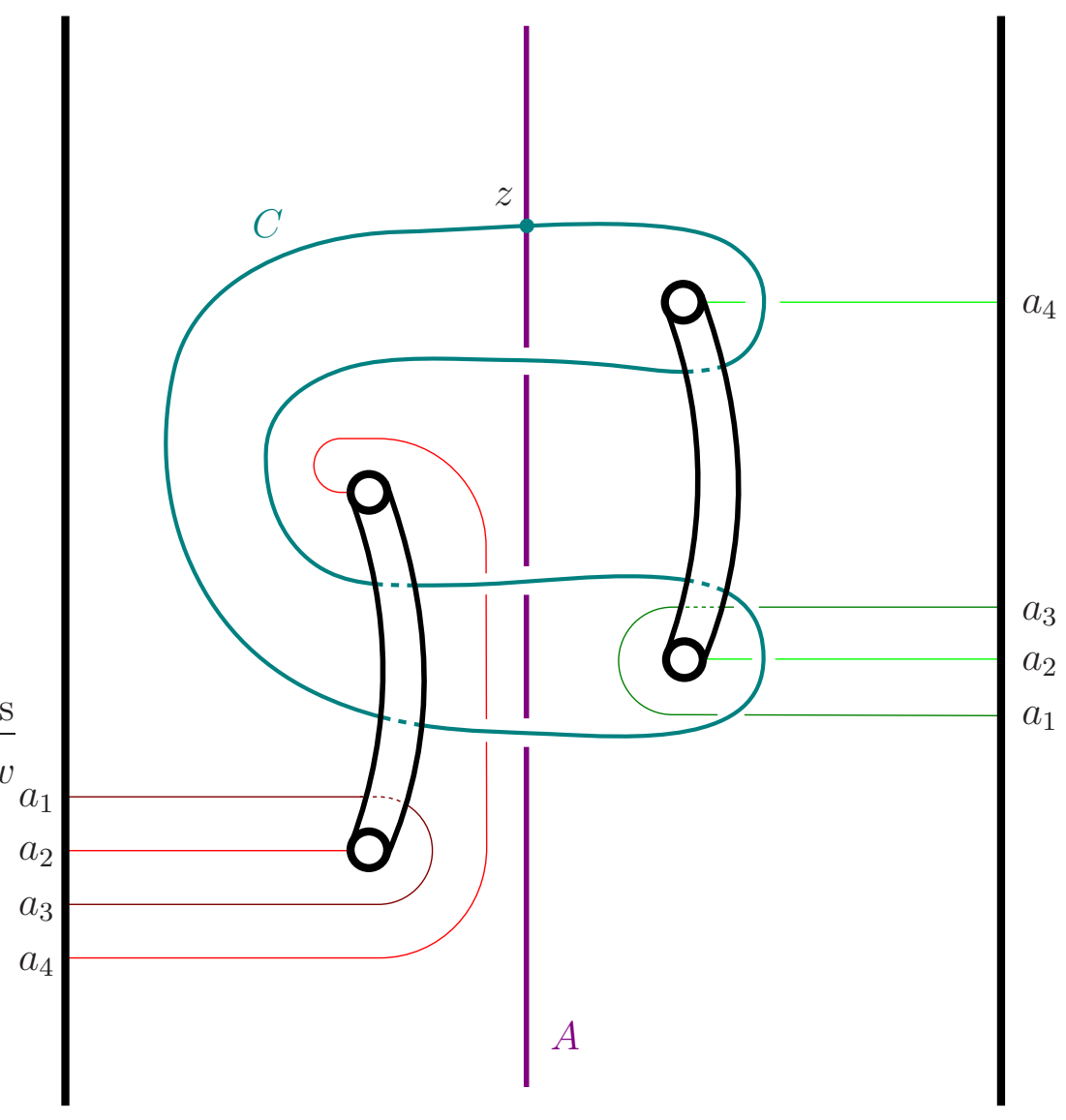

Figure 7. The result of isotoping Figure[6, Each boundary component is identified with $\mathcal{Z}$.

along $A$, which can be seen as $[-\epsilon, \epsilon] \times \mathbb{R} \times(-\infty, 0]$ plus the point at infinity. Then $Y(\mathcal{H})$ is the complement of a two-component unlink $\left(B_{1} \cup B_{2}\right)$ in $S^{3}$, and the $\operatorname{knot} C$ inside $Y(\mathcal{H})$ is $B_{3}$. When we identify each component of $\partial \Sigma$ with $\mathcal{Z}$, we see that the $\alpha$ arc connecting the points $a_{1}$ and $a_{3}$ is a meridian, and the $\alpha$ arc connecting $a_{2}$ and $a_{4}$ is a 0 -framed longitude, as in the definition of $\mathcal{Y}$.

If we try to compute $\widehat{\mathrm{CFAA}}(\mathcal{H}, 0)$ directly, we run into difficulties counting the holomorphic curves, largely because there is a 14-sided region that runs over both handles and shares edges with itself. Instead, it is easier to perform a sequence of isotopies on the $\alpha$ arcs to obtain the diagram $\mathcal{H}^{\prime}$ shown in Figure 8 . While $\mathcal{H}^{\prime}$ is not a nice diagram in the sense of Sarkar and Wang [22], the analysis needed to count the relevant holomorphic curves is vastly simpler. Of course, the drawback is that the number of generators is much larger.

By Theorem 2.3, it suffices to compute $\widehat{\mathrm{CFDD}}\left(\mathcal{H}^{\prime}, 0\right)$, as described previously. Thus, we identify each component of $\partial \Sigma$ with $-\mathcal{Z}$. We now describe this computation.

The bimodule $\widehat{\mathrm{CFDD}}\left(\mathcal{H}^{\prime}, 0\right)$ is a type $D D$ structure over two copies of the torus algebra $\mathcal{A}$. We denote these copies by $\mathcal{A}_{\rho}$ and $\mathcal{A}_{\sigma}$, corresponding to the left and right 


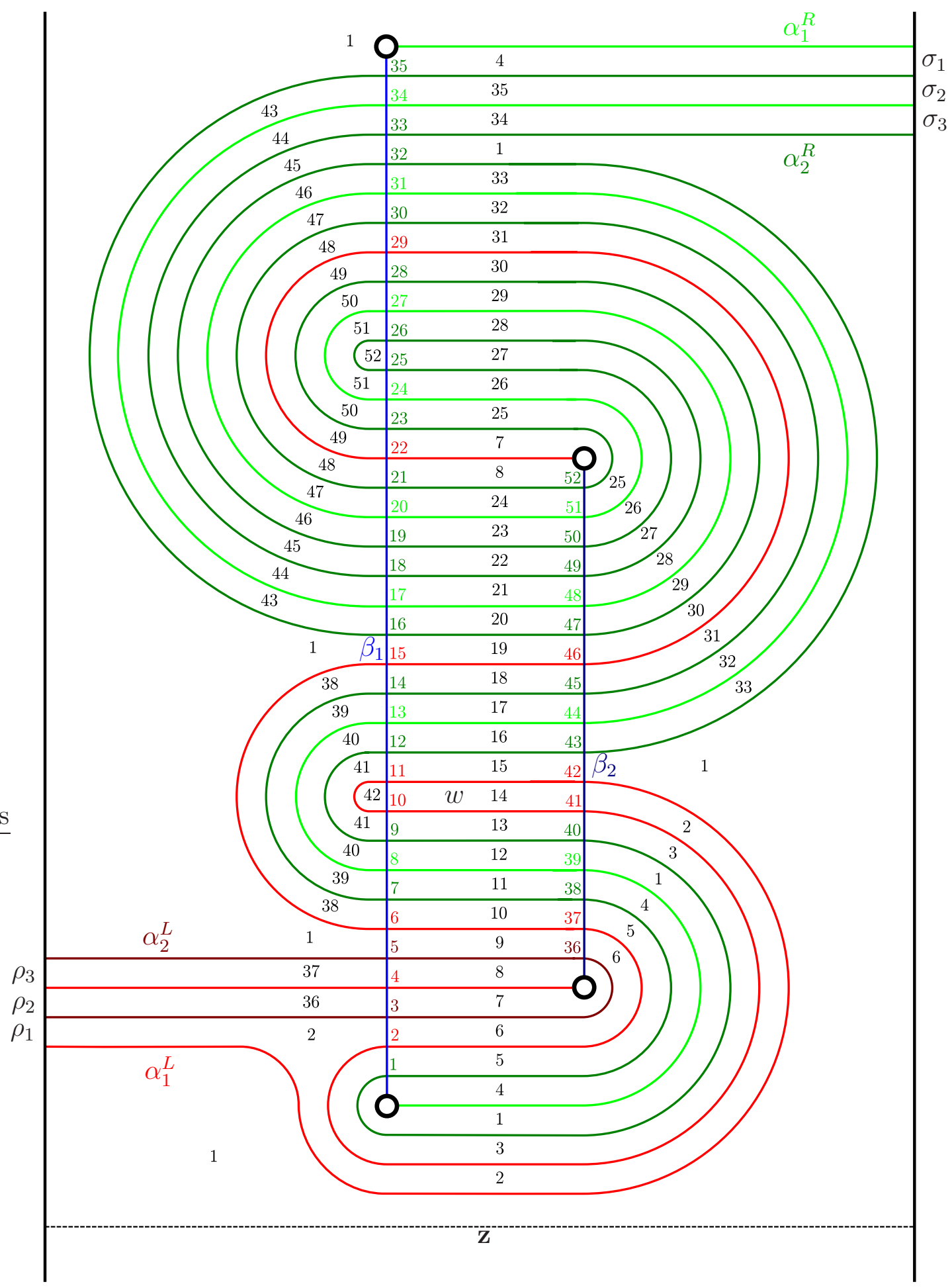

FiguRE 8 . The Heegaard diagram $\mathcal{H}^{\prime}$, with the boundary labeled consistently with the conventions for type $D$ structures. 
boundary components of $\mathcal{H}^{\prime}$. In $\mathcal{A}_{\sigma}$, the Reeb elements are denoted $\sigma_{1}, \sigma_{2}$, etc. The idempotents in $\mathcal{A}_{\rho}$ are denoted $\iota_{0}^{\rho}$ and $\iota_{1}^{\rho}$, and those in $\mathcal{A}_{\sigma}$ are denoted $\iota_{0}^{\sigma}$ and $\iota_{1}^{\sigma}$. The idempotent maps $I_{D}^{\rho}: \mathfrak{S}\left(\mathcal{H}^{\prime}, 0\right) \rightarrow\left\{\iota_{0}^{\rho}, \iota_{1}^{\rho}\right\}$ and $I_{D}^{\sigma}: \mathfrak{S}\left(\mathcal{H}^{\prime}, 0\right) \rightarrow\left\{\iota_{0}^{\sigma}, \iota_{1}^{\sigma}\right\}$ are defined just as in (2.4).

We denote the regions in $\mathcal{H}^{\prime}$ by $R_{1}, \ldots, R_{52}$, as indicated by the black numbers in Figure 8. We label the intersection points of the $\alpha$ and $\beta$ curves $x_{1}, \ldots, x_{52}$, as indicated by the colored numbers. These points are distributed among the various $\alpha$ and $\beta$ circles as follows:

\begin{tabular}{|c|c|c|}
\hline & $\beta_{1}$ & $\beta_{2}$ \\
\hline$\alpha_{1}^{L}$ & $x_{2}, x_{4}, x_{6}, x_{10}, x_{11}, x_{15}, x_{22}, x_{29}$ & $x_{37}, x_{41}, x_{42}, x_{46}$ \\
\hline$\alpha_{2}^{L}$ & $x_{3}, x_{5}$ & $x_{36}$ \\
\hline$\alpha_{1}^{R}$ & $x_{8}, x_{13}, x_{17}, x_{20}, x_{24}, x_{27}, x_{31}, x_{34}$ & $x_{39}, x_{44}, x_{48}, x_{51}$ \\
\hline$\alpha_{2}^{R}$ & $x_{1}, x_{7}, x_{9}, x_{12}, x_{14}, x_{16}, x_{18}, x_{19}$ & $x_{38}, x_{40}, x_{43}, x_{45}$ \\
& $x_{21}, x_{23}, x_{25}, x_{26}, x_{28}, x_{30}, x_{32}, x_{33}, x_{35}$ & $x_{47}, x_{49}, x_{50}, x_{52}$ \\
\hline
\end{tabular}

The underlying vector space for $\widehat{\operatorname{CFDD}}\left(\mathcal{H}^{\prime}, 0\right)$ is generated by the set $\mathfrak{S}\left(\mathcal{H}^{\prime}, 0\right)$, consisting pairs of intersection points with one point on each $\beta$ circle, one point on either $\alpha_{1}^{L}$ or $\alpha_{2}^{L}$, and one point on either $\alpha_{1}^{R}$ or $\alpha_{2}^{R}$. A simple count shows that there are 245 generators.

3.2. Enumerating index-1 positive domains. In order to find all index- 1 positive domains in $\mathcal{H}^{\prime}$, we begin with the following lemma:

Lemma 3.2. For any generators $\mathbf{x}$ and $\mathbf{y}$, the set $\pi_{2}(\mathbf{x}, \mathbf{y})$ is nonempty, and there is at most one domain in $\pi_{2}(\mathbf{x}, \mathbf{y})$ with any prescribed multiplicities in the six boundary regions $\left(R_{2}, R_{4}, R_{34}, R_{35}, R_{36}\right.$, and $\left.R_{37}\right)$.

Proof. For the first statement, the obstruction to $\pi_{2}(\mathbf{x}, \mathbf{y})$ being nonempty is an element $\epsilon(\mathbf{x}, \mathbf{y}) \in H_{1}(Y, \partial Y)$ that is in the image of $H_{1}(Y) \rightarrow H_{1}(Y, \partial Y)$, and this image is trivial since $H_{1}(\partial Y) \rightarrow H_{1}(Y)$ is surjective.

The group of periodic domains in $\mathcal{H}^{\prime}$ is isomorphic to $H_{2}(Y, \partial Y) \cong \mathbb{Z}^{2}$; it is freely generated by

$$
\begin{gathered}
P_{1}=R_{2}+R_{6}+R_{7}-R_{10}-\cdots-R_{13}-R_{15}-\cdots-R_{18}+R_{25}+\cdots+R_{30}+R_{36} \\
\quad-R_{38}-\cdots-R_{41}+R_{49}+\cdots+R_{52} \\
P_{2}=R_{4}+\cdots+R_{11}+R_{17}+\cdots+R_{20}+R_{24}+R_{25}+R_{29}+\cdots+R_{32}+R_{35} \\
\quad-R_{40}-R_{41}-R_{42}-R_{44}-R_{45}-R_{46}-R_{51}-R_{52} .
\end{gathered}
$$

Thus, any nonzero periodic domain has a nonzero multiplicity at either $R_{2}$ or $R_{4}$, so there are no nonzero provincial periodic domains. The uniqueness statement then follows immediately.

We may algorithmically find all the positive domains with index 1 by the following procedure. By Proposition 2.1, the multiplicity of each of the six boundary regions must be 0 or 1 . For each of the $2^{6}$ choices of boundary multiplicities and each pair of generators $\mathbf{x}, \mathbf{y}$ (subject to the idempotent restrictions of Proposition 2.1), we may solve (2.3) to find the unique domain in $\pi_{2}(\mathbf{x}, \mathbf{y})$ with the prescribed boundary 


\begin{tabular}{|l|l|l|l|}
\hline Type of domain & Examples & Quantity & Count for differential? \\
\hline Bigons & $R_{36}, R_{42}$ & 488 & Yes \\
Quadrilaterals & $R_{4}, R_{5}$ & 167 & Yes \\
Domains with a boundary cut & $D_{1}, D_{2}, D_{3}, D_{4}$ & 52 & Yes \\
Domains without a boundary cut & $D_{5}, D_{6}, D_{20}$ & 171 & No \\
Disconnected domains & $D_{7}$ & 37 & No \\
Indecomposable annuli & $D_{8}, D_{9}, D_{10}$ & 35 & Yes \\
Singly decomposable annuli & $D_{11}, D_{12}, D_{13}$ & 18 & No* \\
Doubly decomposable annuli & $D_{14}, D_{15}, D_{16}$ & 7 & No* \\
Good tori & $D_{17}, D_{19}$ & 29 & Yes \\
Conditional tori & $D_{18}$ & 9 & Yes* \\
\hline
\end{tabular}

TABLE 1. Summary of the different types of domains, along with whether or not they count for the differential on $\widehat{\operatorname{CFDD}}\left(\mathcal{H}^{\prime}, 0\right)$. The starred entries in the fourth column hold when the complex structure on $\Sigma$ is sufficiently stretched.

multiplicities, if one exists. We may then list only those solutions which represent positive classes and have index 1 for some compatible $\vec{\rho}$, where the index is computed using (2.5). Specifically, note that if $B$ is domain representing a class in $\pi_{2}(\mathbf{x}, \mathbf{y})$ with boundary multiplicities all 0 or 1 , the quantity $|\vec{\rho}|+\iota(\vec{\rho})$ in $(2.5)$ equals 0 if $B$ is provincial, $\frac{1}{2}$ if it abuts one component of $\partial \Sigma$, and 1 if it abuts both components. The Euler measure of $B$ equals the sum of of the Euler measures of its regions (namely $1-\frac{k}{2}$ for a $2 k$-gon), weighted by their multiplicities. Using a Mathematica computation, we find that there are 1,013 positive index-1 domains satisfying the restrictions of Proposition $2.1^{5}$

We now partition the 1,013 positive domains with index 1 into classes that share the same holomorphic geometry and discuss each case that arises. The results are summarized in Table 1 .

Bigons and quadrilaterals. In the context of closed Heegaard diagrams, Sarkar and Wang [22] showed that in a Heegaard diagram in which every non-basepointed region is either a bigon or a quadrilateral, the domains with Maslov index 1 are precisely the embedded bigons and quadrilaterals that are embedded in the Heegaard diagram, and these all support support a unique holomorphic representatives. (Such a Heegaard diagram is called nice.) Lipshitz, Ozsváth, and Thurston proved an analogous result for bordered diagrams [10, Proposition 8.4], where now we extend the definition of "quadrilateral" to include a region with boundary consisting of one segment of a $\beta$ circle, two segments of $\alpha$ arcs, and one segment of $\partial \Sigma$. The only non-basepointed regions in $\mathcal{H}^{\prime}$ that are not bigons or quadrilaterals are $R_{2}, R_{4}, R_{7}$, and $R_{8}$, which are

\footnotetext{
${ }^{5}$ More precisely, we mean that there are 1,013 tuples $(\mathbf{x}, \mathbf{y}, B)$, where $\mathbf{x}$ and $\mathbf{y}$ are generators and $B \in \pi_{2}(\mathbf{x}, \mathbf{y})$ is a positive class with index 1 . In some cases, the same domain $B$ may be used for different pairs of generators, such as when $B$ is a bigon. We shall use this abuse of terminology throughout this section.
} 

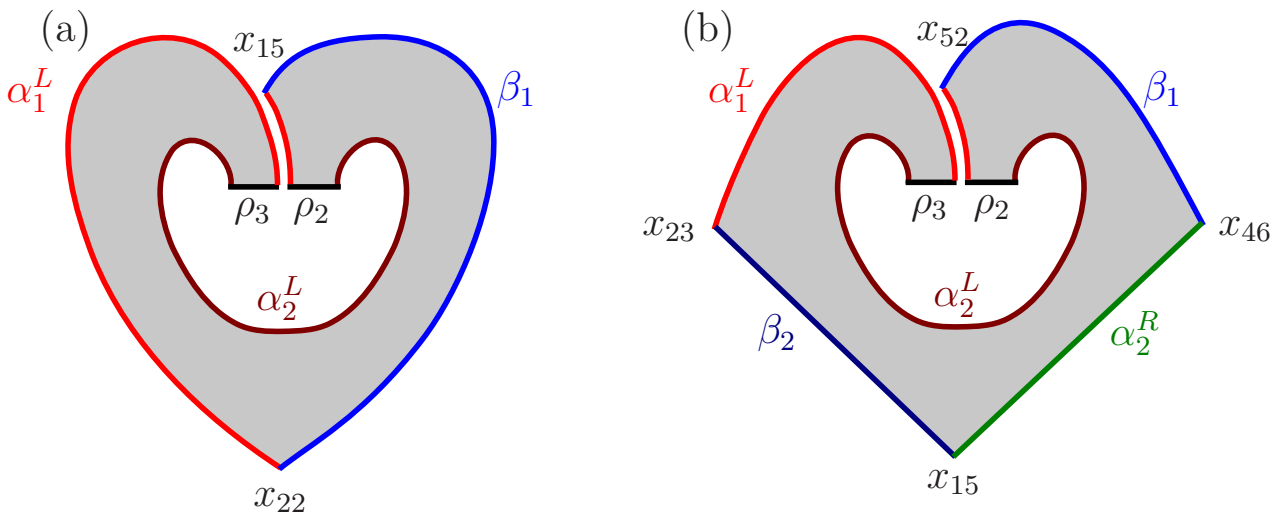

Figure 9. The domains $D_{1}$ (a) and $D_{3}(\mathrm{~b})$.

hexagons. Therefore, any index-1 domain on our list that does not use one of these four regions automatically supports a unique holomorphic representative.

We may easily find several additional families of domains that are embedded bigons or quadrilaterals, perhaps with one or more boundary punctures, which use at least one of the regions $R_{2}, R_{4}, R_{7}$, or $R_{8}$. For instance, $R_{2}+R_{6}+R_{14}+R_{42}$ is a boundarypunctured bigon from $x_{3} x_{i}$ to $x_{2} x_{i}$ (for any $i \in\{38,39,40,43,44,45,47, \ldots, 52\}$ ) with chord marked $\rho_{1}$, and $R_{4}$ is a boundary-punctured rectangle from $x_{35} x_{39}$ to $x_{1} x_{38}$ with a chord marked $\sigma_{1}$.

In total, we find some 488 bigons and 167 quadrilaterals.

Domains with a boundary cut. Let

$$
D_{1}=R_{7}+R_{8}+R_{19}+\cdots+R_{30}+R_{36}+R_{37}+R_{49}+R_{50}+R_{51}+R_{52},
$$

For any $i \in\{38,39,40,43,44,45\} D_{1}$ represents a class in $\pi_{2}\left(x_{15} x_{i}, x_{22} x_{i}\right)$ and has index 1 with respect to the sequence $\left(-\rho_{2},-\rho_{3}\right)$. (If $i \in\{47, \ldots, 52\}$, the index is 3 rather than 1.) To obtain a holomorphic representative of $D_{1}$ compatible with $\left(-\rho_{2},-\rho_{3}\right)$, we cut along $\alpha_{1}^{L}$ all the way to the boundary, as shown in Figure 9. Thus, we parametrize $D_{1}$ as a bigon with two separate boundary punctures rather than as an annulus with a single puncture (which is prohibited by Proposition 2.1). It is then straightforward to see that $D_{1}$ supports a unique holomorphic representative and thus provides a differential $x_{15} x_{i} \stackrel{\rho_{23} \otimes \mathbf{1}}{\longrightarrow} x_{22} x_{i}$ for each $i$ as above. Likewise, for each $j \in\{36,37,41,42,46\}$, the domain

$D_{2}=R_{4}+R_{11}+R_{17}+R_{20}+R_{24}+R_{25}+R_{29}+R_{32}+R_{35}+R_{39}+R_{43}+R_{47}+R_{50}$,

representing a class in $\pi_{2}\left(x_{35} x_{j}, x_{1} x_{j}\right)$, contributes a differential $x_{35} x_{j} \stackrel{1 \otimes \sigma_{12}}{\longrightarrow} x_{1} x_{j}$. In fact, $D_{1}$ and $D_{2}$ are the only domains of this form (so they account for 11 of the 1,013 total classes). 

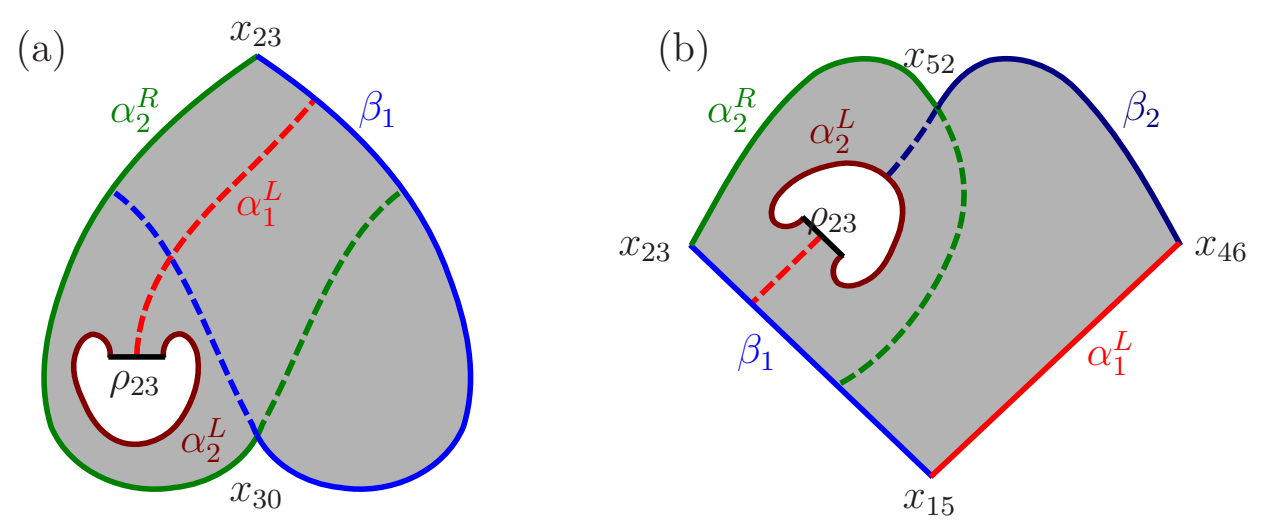

Figure 10. The domains $D_{5}(\mathrm{a})$ and $D_{6}(\mathrm{~b})$.

Similarly, the domains

$$
\begin{aligned}
D_{3}= & R_{7}+R_{8}+R_{31}+R_{36}+R_{37}+R_{48} \\
D_{4}= & D_{2}+R_{4}+R_{5}+R_{10}+R_{11}+R_{17}+R_{18}+R_{24}+R_{25}+R_{29}+R_{30}+R_{32} \\
& \quad+R_{36}+R_{37}+R_{38}+R_{39}+R_{47}+R_{49}+R_{50},
\end{aligned}
$$

respectively represent index- 1 classes in $\pi_{2}\left(x_{22} x_{45}, x_{23} x_{46}\right)$, and $\pi_{2}\left(x_{35} x_{46}, x_{2} x_{48}\right)$. The source curve for $D_{3}$ or $D_{4}$ is a quadrilateral, with two boundary punctures on one $\alpha$ edge mapping to $-\rho_{2}$ and $-\rho_{3}$ and (for $D_{4}$ ) a boundary puncture on the other $\alpha$ edge mapping to $\sigma_{1}$. It is easy to see that these classes all support holomorphic representatives. Thus, we have differentials $x_{15} x_{i} \stackrel{\rho_{23} \otimes \mathbf{1}}{\longrightarrow} x_{22} x_{i}, x_{22} x_{45} \stackrel{\rho_{23} \otimes \mathbf{1}}{\longrightarrow} x_{23} x_{46}$, and $x_{35} x_{46} \stackrel{\rho_{23} \otimes \sigma_{1}}{\longrightarrow} x_{2} x_{48}$. We find 41 domains of this form.

Domains without a boundary cut. Let

$$
\begin{aligned}
& D_{5}=R_{7}+R_{8}+R_{36}+R_{37}+R_{48}+R_{49}+R_{50}+R_{51}+R_{52} \\
& D_{6}=R_{7}+R_{8}+R_{19}+R_{20}+R_{21}+R_{22}+R_{23}+R_{24}+R_{36}+R_{37},
\end{aligned}
$$

illustrated in Figure 10, $D_{5}$ represents a class in $\pi_{2}\left(x_{30} x_{j}, x_{23} x_{j}\right)$ for each $j \in$ $\{37,41,42,46\}$, and $D_{6}$ represents a class in $\pi_{2}\left(x_{15} x_{52}, x_{23} x_{46}\right)$. We cannot cut these domains along $\alpha_{1}^{L}$ as we did with $D_{3}$ and $D_{4}$, since in each case, as we travel along $\alpha_{1}^{L}$ from the intersection point of $\rho_{2} \cap \rho_{3}$, we reach the boundary of $D_{5}$ (resp. $D_{6}$ ) before reaching $x_{j}$ (resp. $x_{46}$ ). Thus, $D_{5}$ and $D_{6}$ cannot admit holomorphic representatives. We find 106 domains like $D_{5}$ and 62 domains like $D_{6}$. (Some of these domains have additional $\sigma_{2}$ or $\sigma_{3}$ punctures on their boundaries, but these do not affect the argument above.)

We also find domains such as

$$
D_{7}=R_{7}+R_{8}+R_{14}+R_{36}+R_{37}+R_{42}
$$

which are the disjoint union of an annulus, one of whose boundary component equals $\alpha_{2}^{L} \cup \rho_{2} \cup \rho_{3}$, and a bigon. Since the $\alpha_{2}^{L} \cup \rho_{2} \cup \rho_{3}$ boundary component of the annulus does not contain a point of either the source or the target generator, there is no way to find a source surface representing $D_{7}$. We find 37 domains of this form. 

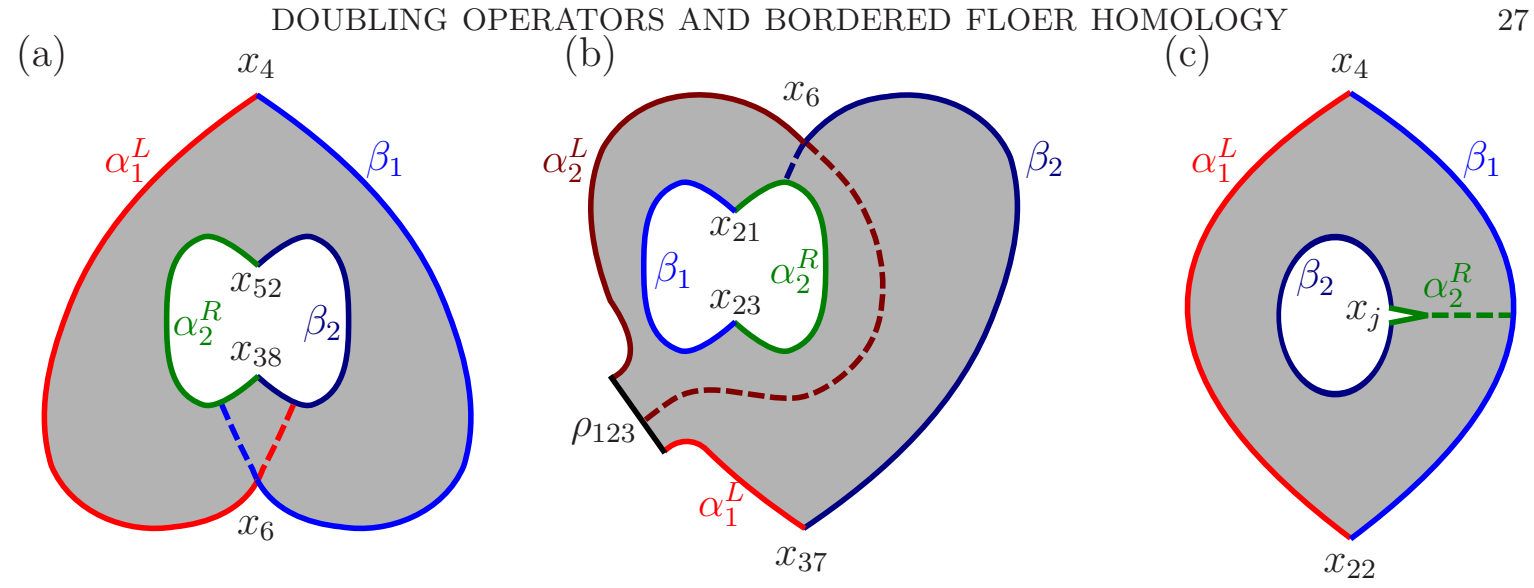

Figure 11 . The indecomposable annuli $D_{8}(\mathrm{a}), D_{9}(\mathrm{~b})$, and $D_{10}$ (c).

Indecomposable annuli. Consider the domains

$$
\begin{aligned}
& D_{8}=R_{8}+R_{9}+R_{10}+R_{18}+R_{31}+R_{38}+R_{48} \in \pi_{2}\left(x_{4} x_{38}, x_{6} x_{52}\right) \\
& D_{9}=R_{2}+R_{6}+R_{7}+R_{8}+R_{14}+R_{36}+R_{37}+R_{42} \in \pi_{2}\left(x_{21} x_{36}, x_{23} x_{37}\right)
\end{aligned}
$$

shown in Figure 11, Each of these domains is topologically an annulus with three convex corners and one concave corner and cannot be decomposed as the composition of an index- 0 annulus and a bigon (in contrast to another family of annuli considered below). As illustrated in Figure 11, we call the boundary component containing the convex corner the outer boundary and the other component the inner boundary. There are a total of eight domains with the geometry of $D_{8}$ and fifteen with the geometry of $D_{9}$.

Lemma 3.3. For any complex structure on $\Sigma$, the moduli spaces $\mathcal{M}\left(D_{8}\right)$ and $\mathcal{M}\left(D_{9}\right)$ each contain an odd number of points. Thus, $D_{8}$ and $D_{9}$ always count for the differential.

Proof. Given a choice of complex structure on $\Sigma$, each domain admits a one-dimensional family of conformal structures, depending on the value of a cut parameter $c \in \mathbb{R}$, where $c<0$ corresponds to cutting along the $\alpha$ curve and $c>0$ corresponds to cutting along the $\beta$ curve. For each value of the cut parameter $c$, let $\theta_{0}(c)\left(\right.$ resp. $\theta_{1}(c)$ ) denote the ratio of the conformal length of the $\alpha$ arc of the outer (resp. inner) boundary to the conformal length of the $\beta$ arc of the outer (resp. inner) boundary. A given conformal structure admits a holomorphic involution if and only if $\theta_{0}(c)=\theta_{1}(c)$, so the number of points in the moduli space of each domain (modulo 2) equals the number of zeros of the function $f(c)=\theta_{0}(c)-\theta_{1}(c)$, which for generic choices of complex structure on $\Sigma$ can be assumed to be transverse to 0 . This number is determined by the limiting behavior of $f(c)$, as follows.

For $D_{8}$, the cut in the $\alpha$ direction approaches the $\beta$ arc of the inner boundary and the cut in the $\beta$ direction approaches the $\alpha$ arc of the inner boundary. Thus, in the limit as we cut in the $\alpha$ direction, $\theta_{0}(c)$ becomes arbitrarily large and $\theta_{1}(c)$ approaches 0 , so $\lim _{c \rightarrow-\infty} f(c)=\infty$. Similarly, as we cut in the $\beta$ direction, $\theta_{0}(c)$ approaches 0 
and $\theta_{1}(c)$ becomes arbitrarily large, so $\lim _{c \rightarrow \infty} f(c)=-\infty$. By transversality and the intermediate value theorem, $f(c)$ has an odd number of zeros.

For $D_{9}$, there is a Reeb chord marked $\rho_{123}$ on the outer boundary. The cut in the $\beta$ direction approaches the $\alpha$ arc of the inner boundary, while the cut in the $\alpha$ direction approaches this boundary puncture. Thus, as we cut in the $\alpha$ direction, $\theta_{0}(c)$ becomes arbitrarily large, while $\theta_{1}(c)$ approaches a finite value. Thus, $\lim _{c \rightarrow-\infty} f(c)=\infty$, while $\lim _{c \rightarrow \infty} f(c)=-\infty$ just as with $D_{8}$.

Similarly, the annular domain

$$
D_{10}=\sum_{i=8}^{24} R_{i}
$$

represents an index-1 class in $\pi_{2}\left(x_{4} x_{j}, x_{22} x_{j}\right)$ for any of the twelve points $x_{j} \in\left(\alpha_{1}^{R} \cup\right.$ $\left.\alpha_{2}^{R}\right) \cap \beta_{2}$. This domain similarly admits a 1-dimensional family of conformal structures given by a cut parameter by $c \in(0, \infty)$. As we increase the cut parameter, the ratio of the length of the $\alpha$ segment of the boundary component containing $x_{j}$ to the $\beta$ segment of the same tends from 0 to infinity, while the same ratio on the opposite boundary component tends from a finite value to 0 . Thus, $D_{10}$ counts for the differential for each choice of $x_{j}$.

Decomposable annuli. We next consider domains whose moduli spaces may depend nontrivially on the choice of complex structure. As a preliminary, let $\eta \subset \Sigma$ be a simple closed curve passing through the regions $R_{1}$, and $R_{4}, \ldots, R_{12}$. For a given complex structure $J$ on $\Sigma$ and $T \in[0, \infty)$, let $J_{T}$ denote the complex structure obtained by "stretching the neck" along $\eta$ by inserting an annulus of width $T$.

Consider the following domains:

$$
\begin{aligned}
& A_{1}=R_{7}+R_{8}+R_{48}+R_{49}+R_{30}+R_{31} \\
& A_{2}=A_{1}+R_{24}+R_{25}+R_{47}+R_{50}+R_{29}+R_{32} \\
& A_{3}=A_{2}+R_{23}+R_{26}+R_{46}+R_{51}+R_{28}+R_{33} \\
& A_{4}=A_{1}+R_{6}+R_{9} \\
& A_{5}=A_{2}+R_{6}+R_{9} \\
& A_{6}=A_{3}+R_{6}+R_{9}
\end{aligned}
$$

Each of these domains is an index- 0 annulus, with one boundary component consisting of a segment of $\beta_{1}$ and a segment of $\alpha_{1}^{L}$ or $\alpha_{2}^{L}$ and the other consisting of a segment of $\beta_{2}$ and a segment of $\alpha_{1}^{R}$ or $\alpha_{2}^{R}$. We call these the two boundary components the outer boundary and inner boundary, respectively. A choice of complex structure $J$ on $\Sigma$ completely determines a conformal structure on each $A_{i}$. Let $\Theta_{0}^{i}(J)$ (resp. $\left(\Theta_{1}^{i}(J)\right)$ ) denote the ratio of the conformal length of the $\alpha$ segment of the inner (resp. outer) boundary of $A_{i}$ to the conformal length of the $\beta$ segment of the inner (resp. outer) boundary. We say that $J$ is sufficiently stretched if $\Theta_{0}^{i}(J)>\Theta_{1}^{i}(J)$ for each $i=1, \ldots, 6$. 

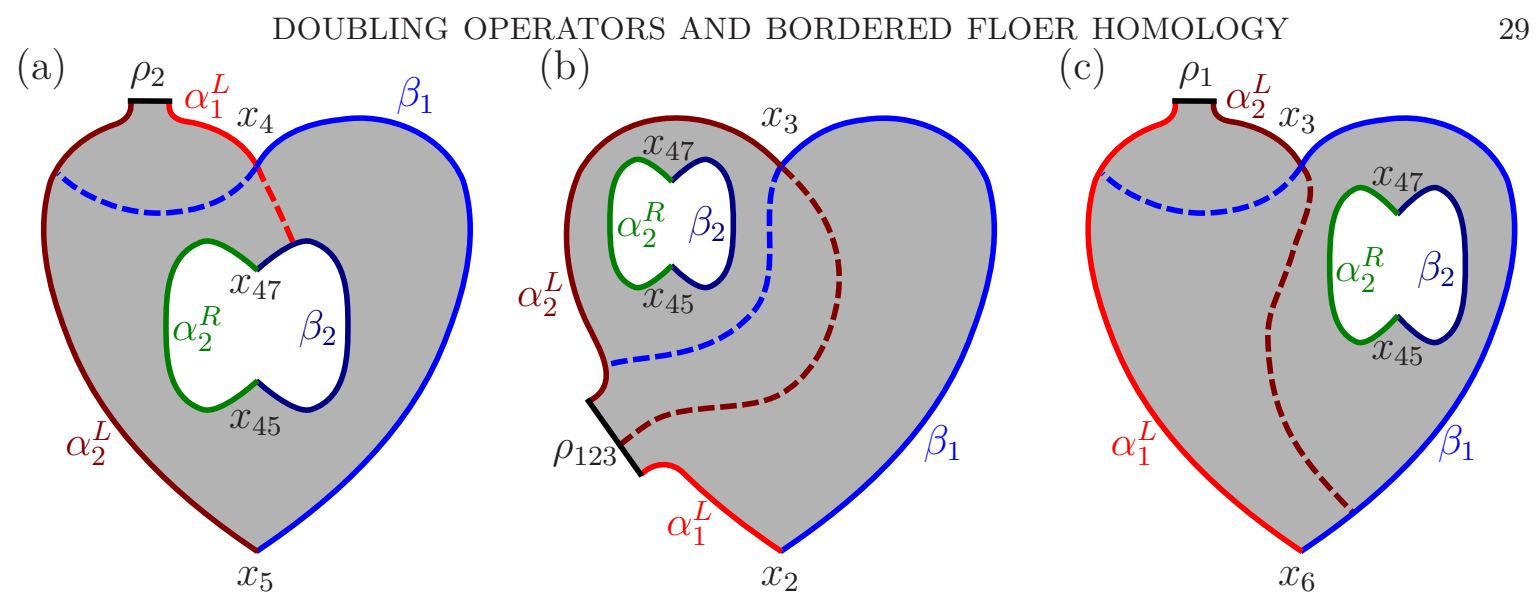

Figure 12. The decomposable annuli $D_{11}$ (a), $D_{14}$ (b), and $D_{15}$ (c).

Lemma 3.4. For any complex structure $J$ on $\Sigma$ there exists a number $T_{0}=T_{0}(J)$ such that for any $T>T_{0}$, the complex structure $J_{T}$ is sufficiently stretched.

Proof. For each $i$, the only intersections of the curve $\eta$ with the boundary of $A_{i}$ are on the $\alpha$ segment of the outer boundary, so stretching the neck along $\eta$ increases the conformal length of that segment relative to the $\beta$ segment of the outer boundary. Therefore, for large values of $T, \Theta_{0}^{i}\left(J_{T}\right)$ can be made arbitrarily large, while $\Theta_{1}^{i}\left(J_{T}\right)$ approaches some finite value.

Consider the index- 1 annuli

$$
\begin{aligned}
D_{11}=A_{1}+R_{36} & \in \pi_{2}\left(x_{4} x_{45}, x_{5} x_{47}\right) \\
D_{12}=A_{1}+R_{37} & \in \pi_{2}\left(x_{3} x_{45}, x_{4} x_{47}\right) \\
D_{13}=A_{1}+R_{25}+ & R_{26}+R_{27}+R_{28}+R_{29}+R_{50}+R_{51}+R_{52} \\
& \in \pi_{2}\left(x_{3} x_{45}, x_{5} x_{52}\right) \\
D_{14}=A_{1}+R_{2}+ & R_{14}+R_{36}+R_{37}+R_{42} \in \pi_{2}\left(x_{3} x_{45}, x_{2} x_{47}\right) \\
D_{15}=A_{1}+R_{2}+ & R_{6}+R_{9}+R_{14}+R_{42} \in \pi_{2}\left(x_{3} x_{45}, x_{6} x_{47}\right) \\
D_{16}=A_{1}+R_{20}+ & R_{24}+R_{25}+R_{29}+R_{32}+R_{35}+R_{43}+R_{47}+R_{50} \\
& \in \pi_{2}\left(x_{3} x_{44}, x_{5} x_{47}\right),
\end{aligned}
$$

some of which are shown in Figure 12, Each of these annuli can be written as a sum of an index-0 annulus and an adjacent bigon, so we call these domains decomposable. It is easy to find eighteen other domains of this form, where we take $A_{2}, \ldots, A_{6}$ in place of $A_{1}$ in (3.2) as applicable. Note that $D_{15}$ and $D_{16}$ can each be decomposed into the sum of an index-0 annulus and an adjacent bigon in a second way as well:

$$
\begin{aligned}
& D_{15}=A_{4}+R_{2}+R_{14}+R_{42} \\
& D_{16}=A_{2}+R_{20}+R_{35}+R_{43},
\end{aligned}
$$

We therefore call these domains doubly decomposable. 
Lemma 3.5. If $J$ is sufficiently stretched, the moduli spaces of all of the decomposable annuli each contain an even number of points. Thus, these domains do not count for the differential.

Proof. We begin with $D_{11}$. Just as with the indecomposable annuli discussed above, there is a 1-dimensional family of conformal structures on $D_{11}$ given by a cut parameter at $x_{4}$. As we cut along $\alpha_{1}^{L}$, the cut approaches the $\beta$ arc of the inner boundary, $\theta_{0}(c)$ becomes arbitrarily large while $\theta_{1}(c)$ approaches 0 , so $\lim _{c \rightarrow-\infty} f(c)=\infty$. On the other hand, cutting along $\beta_{1}$ degenerates $D_{11}$ into $A_{1}$ and a bigon (with a Reeb chord). By Gromov compactness, in the limit as $c \rightarrow \infty$, the ratios $\theta_{0}(c)$ and $\theta_{1}(c)$ approach the corresponding parameters for $A_{1}$, namely $\Theta_{0}^{1}\left(J_{T}\right)$ and $\Theta_{1}^{1}\left(J_{T}\right)$. By Lemma 3.4, if we choose $T$ large enough that $\Theta_{0}^{1}\left(J_{T}\right)>\Theta_{1}^{1}\left(J_{T}\right)$, we see that $\lim _{c \rightarrow \infty} f(c)>0$, so $f(c)$ has an even number of zeroes, as required.

The arguments for $D_{12}, D_{13}$, and $D_{14}$ are very similar. The one modification for $D_{14}$ is that as we cut along $\alpha_{2}^{L}$ at $x_{3}$ out to the boundary puncture, $\theta_{1}(c)$ approaches a finite value that is not necessarily zero, just as we saw with $D_{8}$ above. However, $\theta_{0}(c)$ still approaches $\infty$, so the remainder of the argument carries through unchanged.

For $D_{15}$, cutting along $\alpha_{2}^{L}$ at $x_{3}$ decomposes the domain as in (3.2), while cutting along $\beta_{1}$ decomposes it as in (3.3). Therefore, $\lim _{c \rightarrow-\infty} f(c)=\Theta_{0}^{1}\left(J_{T}\right)-\Theta_{1}^{1}\left(J_{T}\right)$ and $\lim _{c \rightarrow \infty} f(c)=\Theta_{0}^{4}\left(J_{T}\right)-\Theta_{1}^{4}\left(J_{T}\right)$. By Lemma 3.4, we may choose $T$ large enough such that both of these limits are positive numbers, which implies that $f(c)$ has an even number of zeroes. The same analysis goes through for $D_{16}$.

Genus-1 classes. Having analyzed all the classes represented by planar surfaces, we now turn to classes that are represented by surfaces of genus 1 . It is difficult to determine whether these classes support holomorphic representatives using direct conformal geometry arguments as above. Instead, we will look at how these domains arise in the broken flowlines that are the ends of 1-dimensional moduli spaces specifically, the fact the relation $\partial^{2}=0$ and its more complicated $\mathcal{A}_{\infty}$ analogues to deduce the behavior of these domains indirectly. We shall see that knowledge of the planar classes completely determines which of the genus- 1 classes count for the differential.

Consider the domains

$$
\begin{aligned}
& D_{17}=R_{7}+\cdots+R_{24} \in \pi_{2}\left(x_{3} x_{52}, x_{23} x_{36}\right) \\
& D_{18}=R_{7}+\cdots+R_{19}+R_{30}+R_{31}+R_{48}+R_{49} \in \pi_{2}\left(x_{3} x_{45}, x_{16} x_{36}\right)
\end{aligned}
$$

shown in Figure 13. Each of these is represented by an embedded or immersed genus1 surface with one boundary component. Any domain of the form $\sum_{i=a}^{b} R_{i}$, where $a \in\{4,5,6,7\}$ and $b \in\{24, \ldots, 33\}$ are chosen such that the two $\alpha$ segments of the boundary do not lie on the same $\alpha$ curve, has the same holomorphic geometry as $D_{17}$, for a total of 21 domains. Likewise, there are a total of 9 domains with the same geometry as $D_{18}$.

\section{Lemma 3.6.}

(1) For any complex structure $J$ on $\Sigma$, the moduli space $\mathcal{M}_{J}\left(D_{17}\right)$ contains an odd number of points, so $D_{17}$ counts for the differential. 

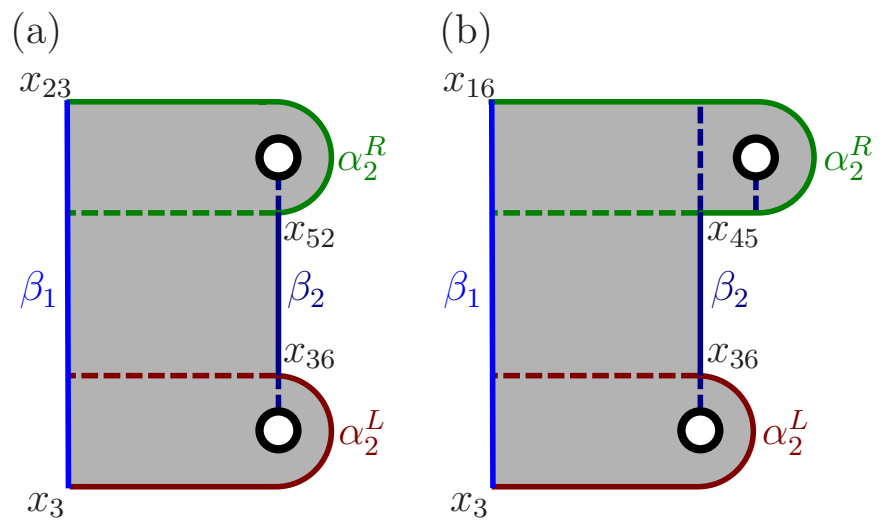

Figure 13. The embedded genus-1 domains $D_{17}$ (a) and $D_{18}$ (b).

(2) If the complex structure $J$ on $\Sigma$ is sufficiently stretched, the moduli space $\mathcal{M}_{J}\left(D_{18}\right)$ contains an odd number of points, so $D_{18}$ counts for the differential.

Proof. Let

$$
\begin{aligned}
& E_{1}=R_{36} \in \pi_{2}\left(x_{4} x_{52}, x_{3} x_{52}\right) \\
& E_{2}=R_{20}+\cdots+R_{29}+R_{50}+R_{51}+R_{52} \in \pi_{2}\left(x_{16} x_{36}, x_{21} x_{36}\right)
\end{aligned}
$$

Each of these domains obviously counts for the differential, and the compositions $E_{1} *$ $D_{17} \in \pi_{2}\left(x_{4} x_{52}, x_{23} x_{36}\right)$ and $D_{18} * E_{2} \in \pi_{2}\left(x_{3} x_{45}, x_{29} x_{52}\right)$ are index-2 positive domains. The moduli spaces $\mathcal{M}\left(E_{1} * E_{17}\right)$ and $\mathcal{M}\left(D_{18} * E_{2}\right)$ are 1-dimensional manifolds, so they each have an even number of ends.

By inspecting the list of all 1,013 index-1 positive domains, it is easy to verify that the only other way to decompose $E_{1} * D_{17}$ into two such domains is as the composition of the indecomposable annulus $D_{10} \in \pi_{2}\left(x_{4} x_{52}, x_{22} x_{52}\right)$ and the boundary-punctured rectangle $R_{7}+R_{36} \in \pi_{2}\left(x_{22} x_{52}, x_{23} x_{36}\right)$, each of which admits a unique holomorphic representative. In order for $\mathcal{M}\left(E_{1} * D_{17}\right)$, which is a 1-dimensional manifold, to have an even number of ends, we see that $D_{17}$ must have an odd number of holomorphic representatives, regardless of the choice of complex structure, and thus must count for the differential.

For $D_{19}$, the situation is slightly more complicated. The composition $D_{18} * E_{2}$ can be split up in two other ways: (a) as the genus-1 domain $R_{7}+\cdots+R_{31} \in$ $\pi_{2}\left(x_{3} x_{45}, x_{30} x_{46}\right)$, which has the same holomorphic geometry as $D_{17}$, composed with the bigon $R_{48}+\cdots+R_{52}$; or (b) as the decomposable annulus $D_{13} \in \pi_{2}\left(x_{3} x_{45}, x_{5} x_{52}\right)$ composed with the rectangle $R_{9}+\cdots+R_{24} \in \pi_{2}\left(x_{5} x_{52}, x_{21}, x_{36}\right)$. Since the moduli space of the composition has an even number of ends, and the composition in (a) provides an odd number of ends by the previous paragraph, it follows that

$$
\# \mathcal{M}\left(D_{18}\right)+\# \mathcal{M}\left(D_{13}\right)+1=0 \quad(\bmod 2)
$$

In other words, $D_{19}$ counts for the differential if and only if $D_{13}$ does not. Thus, if the complex structure is sufficiently stretched, then $\# \mathcal{M}\left(D_{19}\right)=1$ by Lemma 3.5. 


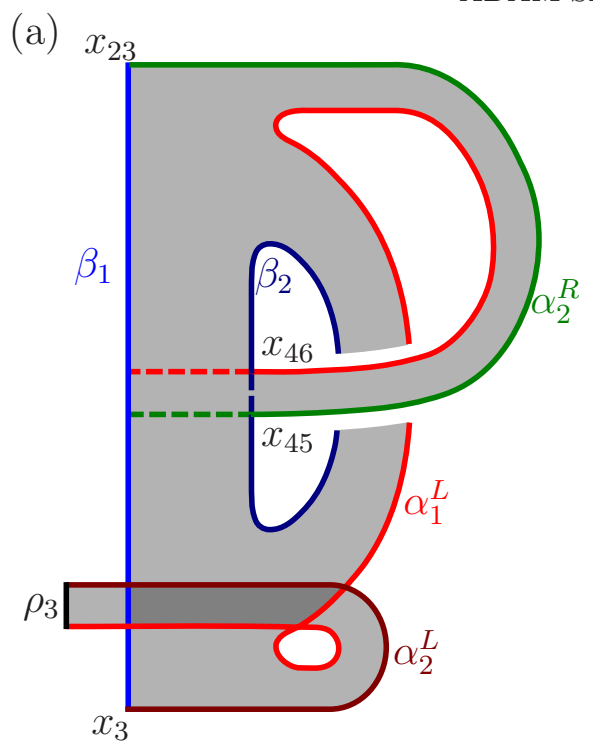

(b)

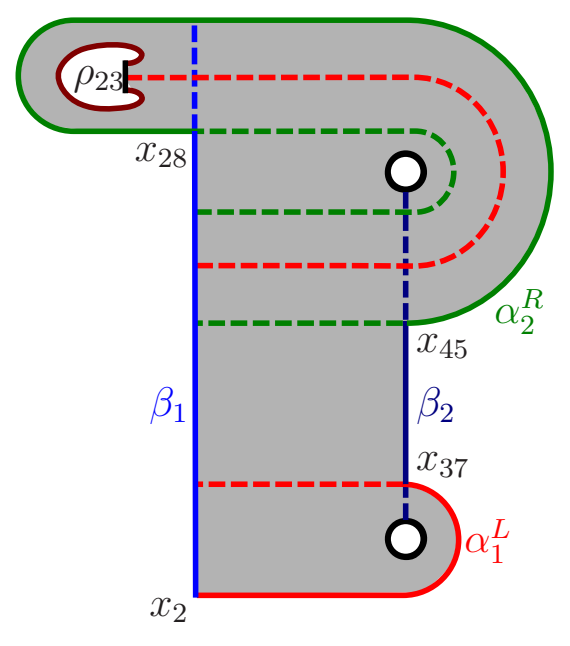

Figure 14 . The immersed genus-1 domains $D_{19}$ (a) and $D_{20}$ (b).

Finally, we consider the domains

$$
\begin{aligned}
& D_{19}=R_{7}+2 R_{8}+R_{9}+R_{10}+\cdots+R_{24}+R_{31}+R_{37}+R_{48} \in \pi_{2}\left(x_{3} x_{45}, x_{23} x_{46}\right) \\
& D_{20}=R_{6}+2 R_{7}+2 R_{8}+R_{9}+\cdots+R_{31}+R_{36}+R_{37}+R_{48}+R_{49},
\end{aligned}
$$

which are represented by immersed genus-1 surfaces as shown in Figure 14. From the figure, we can see that $D_{19}$ is simply an immersed copy of a domain with the same geometry as $D_{17}$ (with a single Reeb chord), so it always counts for the differential. (Alternately, we may give an explicit proof by considering the composition of $D_{19}$ with the rectangle $R_{25}+\cdots+R_{30}$ and arguing as above.) There are 8 domains of this type. On the other hand, $D_{20}$ does not have a cut that can go to the boundary, which would be needed to make it compatible with the sequence $\left(-\rho_{2},-\rho_{3}\right)$, so it does not have any holomorphic representatives. There are two enlargements of $D_{20}$ with the same property.

Combining the results above, we have:

Proposition 3.7. If the complex structure on $\Sigma$ is sufficiently stretched, then 780 of the 1, 013 positive, index-1 domains count for the differential on $\widehat{\mathrm{CFDD}}\left(\mathcal{H}^{\prime}, 0\right)$, as indicated in Table 1 .

Using this list, we may then record the differential on $\widehat{\mathrm{CFDD}}\left(\mathcal{H}^{\prime}, 0\right)$ as a $245 \times 245$ matrix with entries in $\mathcal{A}_{\rho} \otimes \mathcal{A}_{\sigma}$, although for obvious reasons we do not record this matrix here.

By counting the multiplicity at $w$ of each domain (whether or not the domain counts for the differential), we can determine the relative Alexander gradings of all of the generators using (2.8). We find that the generators of $\widehat{\operatorname{CFDD}}\left(\mathcal{H}^{\prime}, 0\right)$ all fall into three consecutive gradings, which for now we arbitrarily declare to be $-1,0$, and 1 . In the end, after we evaluate all tensor products, the symmetry of $\widehat{\mathrm{CFK}}\left(S^{3}, D_{J, s}(K, t)\right)$ 
will show that this was the correct choice. We do not explicitly list all of the gradings here, however.

3.3. Algebraic computation of $\widehat{\mathrm{CFAA}}$. Using our Mathematica package TorusAlgebra.nb, we may apply the edge cancellation algorithm explained in Subsection 2.6 to simplify $\widehat{\mathrm{CFDD}}\left(\mathcal{H}^{\prime}, 0\right)$, canceling only edges that preserve the filtration level. By abuse of notation, we denote the resulting bimodule by $\widehat{\operatorname{CFDD}}\left(\mathcal{Y}, B_{3}, 0\right)$.

Theorem 3.8. The type DD structure $\widehat{\operatorname{CFDD}}\left(\mathcal{Y}, B_{3}, 0\right)$ has a basis $\left\{y_{1}, \ldots, y_{19}\right\}$ with the following properties:

(1) The Alexander gradings of the basis elements are:

$$
A\left(y_{i}\right)= \begin{cases}-1 & i=1 \\ 0 & i=2, \ldots, 10 \\ 1 & i=11, \ldots, 19 .\end{cases}
$$

(2) The associated idempotents in $\mathcal{A}_{\rho}$ and $\mathcal{A}_{\sigma}$ of the generators are:

\begin{tabular}{|c|c|c|}
\hline & $\iota_{0}^{\rho}$ & $\iota_{1}^{\rho}$ \\
\hline$\iota_{0}^{\sigma}$ & $y_{4}, y_{5}, y_{7}, y_{11}, y_{13}, y_{17}, y_{19}$ & $y_{8}, y_{10}, y_{14}, y_{16}$ \\
\hline$\iota_{1}^{\sigma}$ & $y_{3}, y_{6}, y_{12}, y_{18}$ & $y_{1}, y_{2}, y_{9}, y_{15}$ \\
\hline
\end{tabular}

(3) The differential is given by

$$
\delta_{1}\left(y_{i}\right)=\sum_{j=1}^{19} a_{i j} \otimes y_{j}
$$

where $\left(a_{i j}\right)$ is the following matrix:

$\left.\begin{array}{c|ccccccccc|ccccccccc}0 & 0 & 0 & 0 & 0 & 0 & 0 & 0 & 0 & 0 & 0 & 0 & 0 & 0 & 0 & 0 & 0 & 0 & 0 \\ \hline 1 & 0 & 0 & 0 & 0 & 0 & 0 & \sigma_{2} & 0 & 0 & 0 & 0 & 0 & 0 & 0 & 0 & 0 & 0 & 0 \\ \rho_{1} & 0 & 0 & 0 & 0 & 0 & 0 & 0 & 0 & 0 & 0 & 0 & 0 & 0 & 0 & 0 & 0 & 0 & 0 \\ \rho_{1} \sigma_{123} & \rho_{1} \sigma_{3} & \sigma_{3} & 0 & 0 & 0 & 0 & 0 & 0 & 0 & 0 & 0 & 0 & 0 & 0 & 0 & 0 & 0 & 0 \\ 0 & \rho_{3} \sigma_{123}+\rho_{123} \sigma_{3} & 0 & 0 & 0 & \sigma_{3} & 0 & 0 & \rho_{3} \sigma_{123} & \rho_{3} & 0 & 0 & 0 & 0 & 0 & 0 & 0 & 0 & 0 \\ \rho_{123} & 0 & 0 & 0 & 0 & 0 & \sigma_{2} & 0 & \rho_{3} & 0 & 0 & 0 & 0 & 0 & 0 & 0 & 0 & 0 & 0 \\ 0 & 0 & 0 & 0 & 0 & 0 & 0 & \rho_{3} & 0 & 0 & 0 & 0 & 0 & 0 & 0 & 0 & 0 & 0 & 0 \\ 0 & 0 & \rho_{2} \sigma_{1} & 0 & 0 & 0 & 0 & 0 & 0 & 0 & 0 & 0 & 0 & 0 & 0 & 0 & 0 & 0 & 0 \\ 0 & 0 & \rho_{2} & 0 & 0 & 0 & 0 & \sigma_{2} & 0 & 0 & 0 & 0 & 0 & 0 & 0 & 0 & 0 & 0 & 0 \\ \sigma_{123} & 0 & 0 & \rho_{2} & 0 & 0 & 0 & 0 & \sigma_{3} & 0 & 0 & 0 & 0 & 0 & 0 & 0 & 0 & 0 & 0 \\ \hline 0 & \rho_{1} \sigma_{1} & \sigma_{1} & 0 & 0 & 0 & 0 & \rho_{1} & \rho_{1} \sigma_{1} & 0 & 0 & 0 & 0 & 0 & 0 & 0 & 0 & 0 & 0 \\ 0 & \rho_{1} & 1 & 0 & 0 & 0 & 0 & 0 & 0 & 0 & \sigma_{2} & 0 & 0 & 0 & 0 & 0 & 0 & 0 & 0 \\ 0 & 0 & \sigma_{123} & 1 & 0 & 0 & 0 & 0 & 0 & 0 & 0 & \sigma_{3} & 0 & 0 & 0 & 0 & 0 & 0 & 0 \\ 0 & 0 & 0 & 0 & 1 & 0 & 0 & 0 & 0 & 0 & 0 & 0 & 0 & 0 & \sigma_{3} & 0 & 0 & 0 & \rho_{3} \\ 0 & \rho_{123} & 0 & 0 & 0 & 1 & 0 & 0 & 0 & 0 & 0 & 0 & 0 & 0 & 0 & \sigma_{2} & 0 & \rho_{3} & 0 \\ 0 & 0 & 0 & 0 & 0 & 0 & 1 & \rho_{123} & 0 & 0 & 0 & 0 & 0 & 0 & 0 & 0 & \rho_{3} & 0 & 0 \\ 0 & 0 & 0 & 0 & 0 & 0 & 0 & 1 & 0 & 0 & \rho_{2} & 0 & 0 & 0 & 0 & 0 & 0 & 0 & 0 \\ 0 & 0 & 0 & 0 & 0 & 0 & 0 & 0 & 1 & 0 & 0 & \rho_{2} & 0 & 0 & 0 & 0 & \sigma_{2} & 0 & 0 \\ 0 & \sigma_{123} & 0 & 0 & 0 & 0 & 0 & 0 & \sigma_{123} & 1 & 0 & 0 & \rho_{2} & 0 & 0 & 0 & 0 & \sigma_{3} & 0\end{array}\right)$

The block decomposition indicates the filtration levels. 
Finally, to compute $\widehat{\mathrm{CFAA}}\left(\mathcal{Y}, B_{3}, 0\right)$, we use the $A A$ identity bimodule described in Theorem 2.3 ,

$$
\widehat{\mathrm{CFAA}}\left(\mathcal{Y}, B_{3}, 0\right) \simeq \widehat{\mathrm{CFAA}}(\mathbb{I}, 0) \underset{\mathcal{A}_{\sigma}}{\otimes}\left(\widehat{\mathrm{CFAA}}(\mathbb{I}, 0) \underset{\mathcal{A}_{\rho}}{\otimes} \widehat{\mathrm{CFDD}}\left(\mathcal{Y}, B_{3}, 0\right)\right) .
$$

We evaluate this tensor product using our Mathematica package. The filtration on $\widehat{\operatorname{CFDD}}\left(\mathcal{Y}, B_{3}, 0\right)$ induces a filtration on $\widehat{\operatorname{CFAA}}\left(\mathcal{Y}, B_{3}, 0\right)$, and we again use the edge cancellation procedure to reduce the number of generators. For further details on the computation, see Appendix A.

Theorem 3.9. The filtered $A A$-module $\widehat{\mathrm{CFAA}}\left(\mathcal{Y}, B_{3}, 0\right)$ has a basis

$$
\left\{a_{1}, \ldots, a_{5}, b_{1}, \ldots, b_{6}, c_{1}, d_{1}, \ldots, d_{4}, e_{1}, e_{2}, e_{3}\right\}
$$

with the following properties:

(1) The Alexander gradings of the basis elements are:

$$
\begin{aligned}
A\left(c_{1}\right) & =-1 \\
A\left(a_{i}\right)=A\left(d_{i}\right) & =0 \\
A\left(b_{i}\right)=A\left(e_{i}\right) & =1 .
\end{aligned}
$$

(2) The associated idempotents in $\mathcal{A}_{\rho}$ and $\mathcal{A}_{\sigma}$ of the generators are:

\begin{tabular}{|c|c|c|}
\hline & $\iota_{0}^{\rho}$ & $\iota_{1}^{\rho}$ \\
\hline$\iota_{0}^{\sigma}$ & $a_{1}, a_{3}, a_{4}, b_{1}, b_{3}, b_{4}, b_{6}$ & $d_{1}, d_{3}, e_{1}, e_{3}$ \\
\hline$\iota_{1}^{\sigma}$ & $a_{2}, a_{5}, b_{2}, b_{5}$ & $c_{1}, d_{2}, d_{4}, e_{2}$ \\
\hline
\end{tabular}

(3) The $A_{\infty}$ multiplications are presented in the matrices that follow. For $x, y \in$ $\{a, b, c, d, e\}$, the entry in the $i^{\text {th }}$ row and $j^{\text {th }}$ column of the matrix $M_{x y}$ records the multiplications taking $x_{i}$ to $y_{j}$, as described in Subsection 2.1. The matrices $M_{a b}, M_{c b}, M_{c d}, M_{c e}, M_{d b}$, and $M_{d e}$ are necessarily zero because of the Alexander grading.

$$
\begin{aligned}
& M_{a a}=\left(\begin{array}{ccccc}
0 & \sigma_{1} & \sigma_{12} & \rho_{12} & \sigma_{123} \rho_{12}+\sigma_{1} \rho_{3} \rho_{2} \rho_{12} \\
0 & 0 & \sigma_{2} & 0 & \sigma_{23} \rho_{12}+\rho_{12} \\
0 & 0 & 0 & 0 & \sigma_{3} \rho_{12} \\
0 & 0 & 0 & 0 & \sigma_{1} \\
0 & 0 & 0 & 0 & 0
\end{array}\right) \\
& M_{a c}=\left(\begin{array}{l}
\sigma_{123} \rho_{123}+\sigma_{123} \sigma_{23} \rho_{123}+\sigma_{3} \sigma_{2} \sigma_{1} \rho_{123}+\sigma_{1} \sigma_{23} \rho_{3} \rho_{2} \rho_{123} \\
+\sigma_{123} \sigma_{23} \rho_{3} \rho_{2} \rho_{1}+\sigma_{3} \sigma_{2} \sigma_{1} \rho_{3} \rho_{2} \rho_{1}+\sigma_{1} \sigma_{23} \rho_{3} \rho_{2} \rho_{3} \rho_{2} \rho_{1} \\
\sigma_{23} \rho_{123}+\rho_{3} \rho_{2} \rho_{1}+\sigma_{23} \sigma_{23} \rho_{123}+\sigma_{23} \sigma_{23} \rho_{3} \rho_{2} \rho_{1} \\
\sigma_{3} \sigma_{23} \rho_{123}+\sigma_{3} \rho_{3} \rho_{2} \rho_{1}+\sigma_{3} \sigma_{23} \rho_{3} \rho_{2} \rho_{1} \\
\sigma_{123} \rho_{3}+\sigma_{3} \sigma_{2} \sigma_{1} \rho_{3} \\
\sigma_{23} \rho_{3}
\end{array}\right) \\
& M_{a d}=\left(\begin{array}{llll}
\rho_{1} & 0 & \multicolumn{1}{c}{\sigma_{12} \rho_{123}+\sigma_{123} \sigma_{2} \rho_{123}+\sigma_{12} \rho_{3} \rho_{2} \rho_{1}+\sigma_{1} \sigma_{2} \rho_{3} \rho_{2} \rho_{123}} & \\
0 & \rho_{1} & \sigma_{2} \rho_{123}+\sigma_{23} \sigma_{2} \rho_{123}+\sigma_{2} \rho_{3} \rho_{2} \rho_{1}+\sigma_{23} \sigma_{2} \rho_{3} \rho_{2} \rho_{1} & \sigma_{123} \rho_{1}+\sigma_{1} \rho_{3} \rho_{2} \rho_{1} \\
0 & 0 & \sigma_{3} \sigma_{2} \rho_{123}+\sigma_{3} \sigma_{2} \rho_{3} \rho_{2} \rho_{1}+\rho_{1} & \sigma_{23} \rho_{1} \\
0 & 0 & \sigma_{12} \rho_{3} & \sigma_{3} \rho_{1} \\
0 & 0 & \sigma_{2} \rho_{3} & 0 \\
& \quad & 0
\end{array}\right)
\end{aligned}
$$




$$
\begin{aligned}
& M_{b a}=\left(\begin{array}{ccccc}
1 & 0 & 0 & \sigma_{3} \sigma_{2} \rho_{123} \rho_{2}+\sigma_{3} \sigma_{2} \rho_{3} \rho_{2} \rho_{1} \rho_{2} & \sigma_{123} \rho_{3} \rho_{2} \rho_{12}+\sigma_{1} \rho_{3} \rho_{2} \rho_{3} \rho_{2} \rho_{12} \\
0 & 1 & 0 & 0 & \rho_{3} \rho_{2} \rho_{12}+\sigma_{23} \rho_{3} \rho_{2} \rho_{12} \\
0 & 0 & 1 & 0 & \sigma_{3} \rho_{3} \rho_{2} \rho_{12} \\
0 & 0 & 0 & 1+\sigma_{3} \sigma_{2} \rho_{3} \rho_{2} & \sigma_{123} \\
0 & 0 & 0 & 0 & 1+\sigma_{23} \\
0 & 0 & 0 & 0 & \sigma_{3}
\end{array}\right) \\
& M_{b b}=\left(\begin{array}{cccccc}
0 & \sigma_{1} & \sigma_{12} & \rho_{12} & 0 & 0 \\
0 & 0 & \sigma_{2} & 0 & \rho_{12} & 0 \\
0 & 0 & 0 & 0 & 0 & \rho_{12} \\
0 & 0 & 0 & 0 & \sigma_{1} & \sigma_{12} \\
0 & 0 & 0 & 0 & 0 & \sigma_{2} \\
0 & 0 & 0 & 0 & 0 & 0
\end{array}\right) \\
& M_{b c}=\left(\begin{array}{c}
\sigma_{3} \sigma_{2} \sigma_{123} \rho_{123} \rho_{23}+\sigma_{123} \sigma_{23} \rho_{3} \rho_{2} \rho_{123}+\sigma_{123} \rho_{3} \rho_{2} \rho_{3} \rho_{2} \rho_{1}+\sigma_{1} \sigma_{23} \rho_{3} \rho_{2} \rho_{3} \rho_{2} \rho_{123} \\
+\sigma_{3} \sigma_{2} \sigma_{3} \sigma_{2} \sigma_{1} \rho_{123} \rho_{23}+\sigma_{3} \sigma_{2} \sigma_{123} \rho_{3} \rho_{2} \rho_{1} \rho_{23}+\sigma_{123} \sigma_{23} \rho_{3} \rho_{2} \rho_{3} \rho_{2} \rho_{1} \\
+\sigma_{1} \sigma_{23} \rho_{3} \rho_{2} \rho_{3} \rho_{2} \rho_{3} \rho_{2} \rho_{1}+\sigma_{3} \sigma_{2} \sigma_{3} \sigma_{2} \sigma_{1} \rho_{3} \rho_{2} \rho_{1} \rho_{23} \\
\sigma_{23} \rho_{3} \rho_{2} \rho_{123}+\rho_{3} \rho_{2} \rho_{3} \rho_{2} \rho_{1}+\sigma_{23} \sigma_{23} \rho_{3} \rho_{2} \rho_{123}+\sigma_{23} \sigma_{23} \rho_{3} \rho_{2} \rho_{3} \rho_{2} \rho_{1} \\
\sigma_{3} \sigma_{23} \rho_{3} \rho_{2} \rho_{123}+\sigma_{3} \rho_{3} \rho_{2} \rho_{3} \rho_{2} \rho_{1}+\sigma_{3} \sigma_{23} \rho_{3} \rho_{2} \rho_{3} \rho_{2} \rho_{1} \\
\sigma_{3} \sigma_{2} \sigma_{123} \rho_{3} \rho_{23}+\sigma_{3} \sigma_{2} \sigma_{3} \sigma_{2} \sigma_{1} \rho_{3} \rho_{23} \\
0 \\
0
\end{array}\right)
\end{aligned}
$$

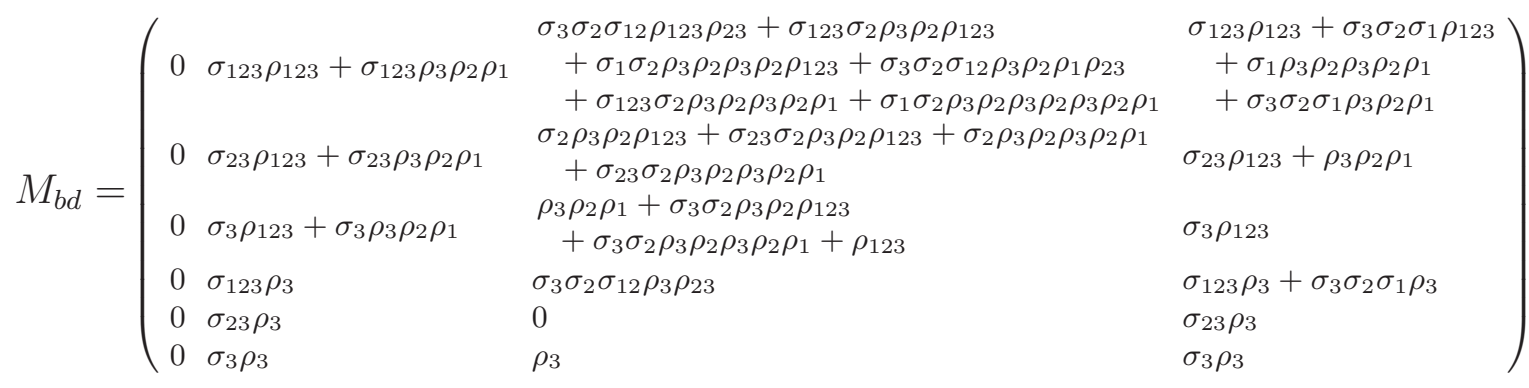

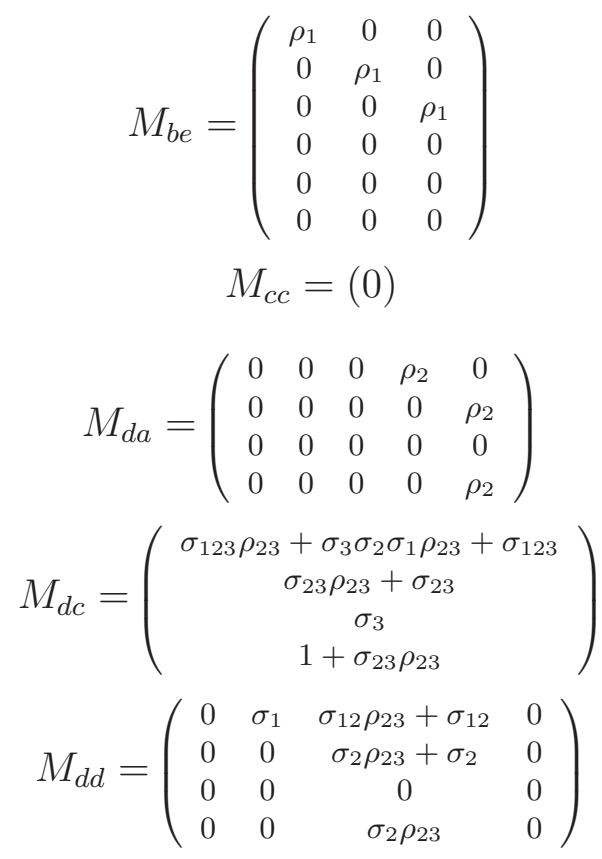




$$
\begin{aligned}
& M_{e a}=\left(\begin{array}{ccccc}
0 & 0 & 0 & \sigma_{3} \sigma_{2} \rho_{23} \rho_{2} & 0 \\
0 & 0 & 0 & 0 & 0 \\
0 & 0 & 0 & 0 & 0
\end{array}\right) \\
& M_{e b}=\left(\begin{array}{cccccc}
0 & 0 & 0 & \rho_{2} & 0 & 0 \\
0 & 0 & 0 & 0 & \rho_{2} & 0 \\
0 & 0 & 0 & 0 & 0 & \rho_{2}
\end{array}\right) \\
& M_{e c}=\left(\begin{array}{c}
\sigma_{3} \sigma_{2} \sigma_{123} \rho_{23} \rho_{23} \\
0 \\
0
\end{array}\right) \\
& M_{e d}=\left(\begin{array}{cccc}
1 & \sigma_{123} \rho_{23} & \sigma_{3} \sigma_{2} \sigma_{12} \rho_{23} \rho_{23} & \sigma_{123} \rho_{23}+\sigma_{3} \sigma_{2} \sigma_{1} \rho_{23}+\sigma_{123} \\
0 & 1+\sigma_{23} \rho_{23} & 0 & \sigma_{23} \rho_{23}+\sigma_{23} \\
0 & \sigma_{3} \rho_{23} & 1+\rho_{23} & \sigma_{3} \rho_{23}+\sigma_{3}
\end{array}\right) \\
& M_{e e}=\left(\begin{array}{ccc}
0 & \sigma_{1} & \sigma_{12} \\
0 & 0 & \sigma_{2} \\
0 & 0 & 0
\end{array}\right)
\end{aligned}
$$

Because we are ultimately interested in the tensor product of $\widehat{\mathrm{CFAA}}\left(\mathcal{Y}, B_{3}, 0\right)$ with $\widehat{\mathrm{CFD}}\left(\mathcal{X}_{J}^{s}\right)$ and $\widehat{\mathrm{CFD}}\left(\mathcal{X}_{K}^{t}\right)$, we may disregard any higher multiplication that uses sequences of algebra elements that cannot occur in these type $D$ structures. Specifically, by Proposition 2.4, we may disregard any sequence containing $\rho_{2} \rho_{3}, \rho_{1} \rho_{2}, \rho_{1} \rho_{23}, \sigma_{2} \sigma_{3}$, $\sigma_{1} \sigma_{2}$, or $\sigma_{1} \sigma_{23}$. Accordingly, for the discussion that follows, we may replace $M_{a c}, M_{a d}$, $M_{b a}, M_{b c}$, and $M_{b d}$ with the following:

$$
\begin{aligned}
& M_{a c}^{\prime}=\left(\begin{array}{c}
\sigma_{123} \rho_{123}+\sigma_{123} \sigma_{23} \rho_{123}+\sigma_{3} \sigma_{2} \sigma_{1} \rho_{123}+\sigma_{123} \sigma_{23} \rho_{3} \rho_{2} \rho_{1}+\sigma_{3} \sigma_{2} \sigma_{1} \rho_{3} \rho_{2} \rho_{1} \\
\sigma_{23} \rho_{123}+\rho_{3} \rho_{2} \rho_{1}+\sigma_{23} \sigma_{23} \rho_{123}+\sigma_{23} \sigma_{23} \rho_{3} \rho_{2} \rho_{1} \\
\sigma_{3} \sigma_{23} \rho_{123}+\sigma_{3} \rho_{3} \rho_{2} \rho_{1}+\sigma_{3} \sigma_{23} \rho_{3} \rho_{2} \rho_{1} \\
\sigma_{123} \rho_{3}+\sigma_{3} \sigma_{2} \sigma_{1} \rho_{3} \\
\sigma_{23} \rho_{3}
\end{array}\right) \\
& M_{a d}^{\prime}=\left(\begin{array}{cccc}
\rho_{1} & 0 & \sigma_{12} \rho_{123}+\sigma_{123} \sigma_{2} \rho_{123}+\sigma_{12} \rho_{3} \rho_{2} \rho_{1}+\sigma_{123} \sigma_{2} \rho_{3} \rho_{2} \rho_{1} & \sigma_{123} \rho_{1}+\sigma_{1} \rho_{3} \rho_{2} \rho_{1} \\
0 & \rho_{1} & \sigma_{2} \rho_{123}+\sigma_{23} \sigma_{2} \rho_{123}+\sigma_{2} \rho_{3} \rho_{2} \rho_{1}+\sigma_{23} \sigma_{2} \rho_{3} \rho_{2} \rho_{1} & \sigma_{23} \rho_{1} \\
0 & 0 & \sigma_{3} \sigma_{2} \rho_{123}+\sigma_{3} \sigma_{2} \rho_{3} \rho_{2} \rho_{1}+\rho_{1} & \sigma_{3} \rho_{1} \\
0 & 0 & \sigma_{12} \rho_{3} & 0 \\
0 & 0 & \sigma_{2} \rho_{3} & 0
\end{array}\right) \\
& M_{b a}^{\prime}=\left(\begin{array}{ccccc}
1 & 0 & 0 & \sigma_{3} \sigma_{2} \rho_{123} \rho_{2} & \sigma_{123} \rho_{3} \rho_{2} \rho_{12} \\
0 & 1 & 0 & 0 & \rho_{3} \rho_{2} \rho_{12}+\sigma_{23} \rho_{3} \rho_{2} \rho_{12} \\
0 & 0 & 1 & 0 & \sigma_{3} \rho_{3} \rho_{2} \rho_{12} \\
0 & 0 & 0 & 1+\sigma_{3} \sigma_{2} \rho_{3} \rho_{2} & \sigma_{123} \\
0 & 0 & 0 & 0 & 1+\sigma_{23} \\
0 & 0 & 0 & 0 & \sigma_{3}
\end{array}\right) \\
& M_{b c}^{\prime}=\left(\begin{array}{c}
\sigma_{3} \sigma_{2} \sigma_{123} \rho_{123} \rho_{23}+\sigma_{123} \sigma_{23} \rho_{3} \rho_{2} \rho_{123} \\
\sigma_{23} \rho_{3} \rho_{2} \rho_{123}+\sigma_{23} \sigma_{23} \rho_{3} \rho_{2} \rho_{123} \\
\sigma_{3} \sigma_{23} \rho_{3} \rho_{2} \rho_{123} \\
\sigma_{3} \sigma_{2} \sigma_{123} \rho_{3} \rho_{23} \\
0 \\
0
\end{array}\right)
\end{aligned}
$$




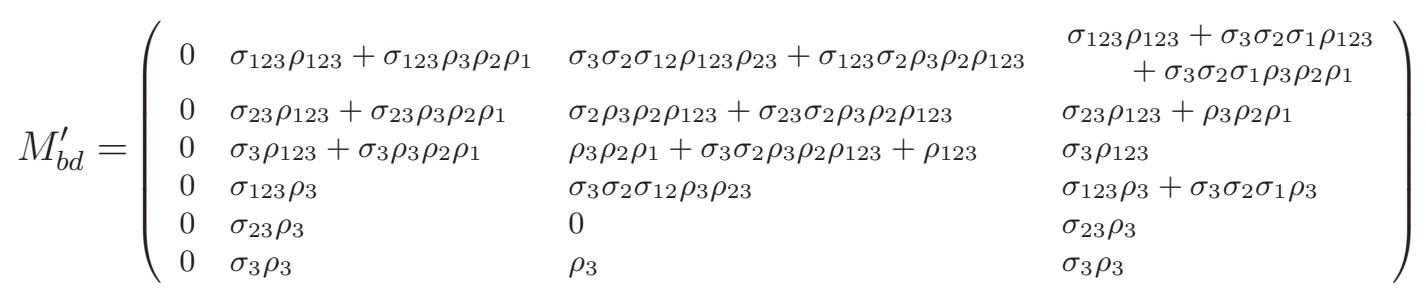

\section{Evaluation of the tensor PROduct}

Using the computation of $\widehat{\operatorname{CFAA}}\left(\mathcal{Y}, B_{3}, 0\right)$ given in the previous section, we may now compute the double tensor product

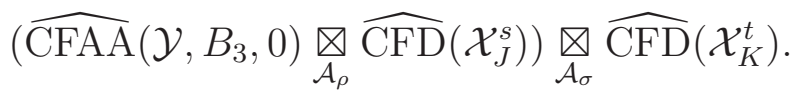

In what follows, we evaluate the tensor product over $\mathcal{A}_{\rho}$ and simplify the resulting filtered type $A$ module before evaluating the tensor product over $\mathcal{A}_{\sigma}$. Then we use the edge cancellation algorithm to compute $\tau\left(D_{J, s}(K, t)\right)$. As a reminder, we restate the main theorem:

$$
\tau\left(D_{J, s}(K, t)\right)= \begin{cases}1 & s<2 \tau(J) \text { and } t<2 \tau(K) \\ -1 & s>2 \tau(J) \text { and } t>2 \tau(K) \\ 0 & \text { otherwise. }\end{cases}
$$

Notice that it suffices to consider only the cases where $s \leq 2 \tau(J)$, since if $s>2 \tau(J)$, the behavior of $\tau$ under mirroring implies:

$$
\begin{aligned}
\tau\left(D_{J, s}(K, t)\right) & =-\tau\left(\overline{D_{J, s}(K, t)}\right) \\
& =-\tau\left(D_{\bar{J},-s}(\bar{K},-t)\right) \\
& = \begin{cases}-1 & -t<2 \tau(\bar{K}) \\
0 & -t \geq 2 \tau(\bar{K})\end{cases} \\
& = \begin{cases}-1 & t>-2 \tau(K) \\
0 & t \leq-2 \tau(K)\end{cases}
\end{aligned}
$$

With only slightly more bookkeeping, we could also write down a formula for the knot Floer homology groups $\widehat{\operatorname{HFK}}\left(D_{J, s}(K, t)\right)$, but since we are primarily interested in the value of $\tau$ and its applications to knot and link concordance, we do not bother to do that here.

4.1. Tensor product over $\mathcal{A}_{\rho}$. Let $\mathcal{V}$ denote the bordered solid torus obtained by gluing together $\mathcal{Y}$ and $\mathcal{X}_{J}^{s}$, and let $D_{J, s}$ denote the image of the knot $B_{3}$ in the union. By the gluing theorem, $\widehat{\operatorname{CFA}}\left(\mathcal{V}, D_{J, s}\right) \simeq \widehat{\operatorname{CFAA}}\left(\mathcal{Y}, B_{3}, 0\right) \underset{\mathcal{A}_{\rho}}{\otimes} \widehat{\operatorname{CFD}}\left(\mathcal{X}_{J}^{s}\right)$. We shall describe this tensor product as a direct sum of subspaces corresponding to the stable and unstable chains in $\widehat{\mathrm{CFD}}\left(\mathcal{X}_{J}^{s}\right)$. This decomposition will not be a direct sum of $\mathcal{A}_{\infty}$ modules, but we will be able to keep track of the few multiplications that do 
not respect the decomposition, and ultimately they will not affect the computation of $\tau\left(D_{J, s}(K, t)\right)$.

The generators of $\iota_{1} \widehat{\mathrm{CFD}}\left(\mathcal{X}_{J}^{s}\right)$ all lie in the interiors of the chains, so the corresponding generators of the tensor product can be grouped in a natural way, but it is not obvious a priori how to divide up the generators coming from $\iota_{0} \widehat{\mathrm{CFD}}\left(\mathcal{X}_{J}^{s}\right)$. Consider the two specified bases for $\iota_{0} \widehat{\mathrm{CFD}}\left(\mathcal{X}_{J}^{s}\right):\left\{\eta_{0}, \ldots, \eta_{2 n}\right\}$ and $\left\{\xi_{0}, \ldots, \xi_{2 n}\right\}$. Depending on the structure of the unstable chain, the generators $\xi_{i}$ have outgoing arrows labeled $\rho_{1}$, $\rho_{12}$, or $\rho_{123}$, while the $\eta_{i}$ have outgoing arrows labeled $\rho_{3}$ and incoming arrows labeled $\rho_{2}$ or $\rho_{12}$. Accordingly, we should try to pair the generators of $\widehat{\operatorname{CFAA}}\left(\mathcal{Y}, B_{3}, 0\right) \iota_{0}$ with the $\xi_{i}$ or $\eta_{i}$ depending on whether they have outgoing $\rho_{1}, \rho_{12}$, and $\rho_{123}$ arrows or outgoing $\rho_{3}$ s and incoming $\rho_{2}$ s. If we consider only the $\mathcal{A}_{\infty}$ maps in $\overline{\operatorname{CFAA}}\left(\mathcal{Y}, B_{3}, 0\right)$ that use a single element of $\mathcal{A}_{\rho}$, we notice that each of the generators $a_{1}, \ldots, a_{5}$ and $b_{1}, \ldots, b_{6}$ satisfies exactly one of these conditions. Specifically, define the following subspaces of $\widehat{\mathrm{CFAA}}\left(\mathcal{Y}, B_{3}, 0\right) \underset{\mathcal{A}_{\rho}}{\otimes} \widehat{\mathrm{CFD}}\left(\mathcal{X}_{J}^{s}\right)$ :

$$
\begin{aligned}
P_{\text {vert }}^{j}= & \left\langle a_{1}, a_{2}, a_{3}, b_{1}, b_{2}, b_{3}\right\rangle \otimes\left\langle\xi_{2 j-1}, \xi_{2 j}\right\rangle \\
& +\left\langle c_{1}, d_{1}, d_{2}, d_{3}, d_{4}, e_{1}, e_{2}, e_{3}\right\rangle \otimes\left\langle\kappa_{i}^{j} \mid 1 \leq i \leq k_{j}\right\rangle \\
P_{\text {hor }}^{j}= & \left\langle a_{4}, a_{5}, b_{4}, b_{5}, b_{6}\right\rangle \otimes\left\langle\eta_{2 j-1}, \eta_{2 j}\right\rangle \\
& +\left\langle c_{1}, d_{1}, d_{2}, d_{3}, d_{4}, e_{1}, e_{2}, e_{3}\right\rangle \otimes\left\langle\lambda_{i}^{j} \mid 1 \leq i \leq l_{j}\right\rangle \\
P_{\text {unst }}= & \left\langle a_{1}, a_{2}, a_{3}, b_{1}, b_{2}, b_{3}\right\rangle \otimes\left\langle\xi_{0}\right\rangle \\
& +\left\langle a_{4}, a_{5}, b_{4}, b_{5}, b_{6}\right\rangle \otimes\left\langle\eta_{0}\right\rangle \\
& +\left\langle c_{1}, d_{1}, d_{2}, d_{3}, d_{4}, e_{1}, e_{2}, e_{3}\right\rangle \otimes\left\langle\lambda_{i} \mid 1 \leq i \leq r\right\rangle .
\end{aligned}
$$

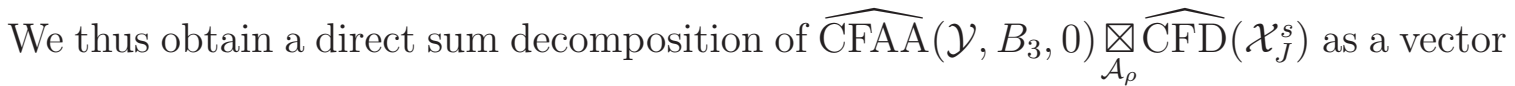
space:

$$
\widehat{\mathrm{CFAA}}\left(\mathcal{Y}, B_{3}, 0\right) \underset{\mathcal{A}_{\rho}}{\otimes} \widehat{\operatorname{CFD}}\left(\mathcal{X}_{J}^{s}\right)=\bigoplus_{j=1}^{n} P_{\text {vert }}^{j} \oplus \bigoplus_{j=1}^{n} P_{\text {hor }}^{j} \oplus P_{\text {unst }} .
$$

By inspecting the matrices $M_{x y}$, we see that any $\mathcal{A}_{\infty}$ multiplication on the tensor product that comes from a multiplication in $\widehat{\mathrm{CFAA}}\left(\mathcal{Y}, B_{3}, 0\right)$ that uses at most one element of $\mathcal{A}_{\rho}$ preserves this decomposition. These multiplications are illustrated in Figures 15 through [18. In these and subsequent figures, the dashed arrows represent repeated sections. For instance, the dashed arrow from $e_{1} \kappa_{1}^{j}$ to $d_{2} \kappa_{k_{j}}^{j}$ in Figure [15] means that there are multiplications $e_{1} \kappa_{i}^{j} \stackrel{\sigma_{123}}{\longrightarrow} d_{2} \kappa_{i+1}^{j}$ for each $i=1, \ldots, k_{j}-1$. The Alexander filtration is indicated by horizontal position, increasing from left to right.

In addition, there are a few more multiplications that preserve the splitting, coming from multiplications in $\widehat{\operatorname{CFAA}}\left(\mathcal{Y}, B_{3}, 0\right)$ that use sequences like $\rho_{3} \rho_{2}, \rho_{3} \rho_{23}$, or $\rho_{23} \rho_{23}$. These multiplications are not shown in Figures 15 through 18 . They are as follows: 


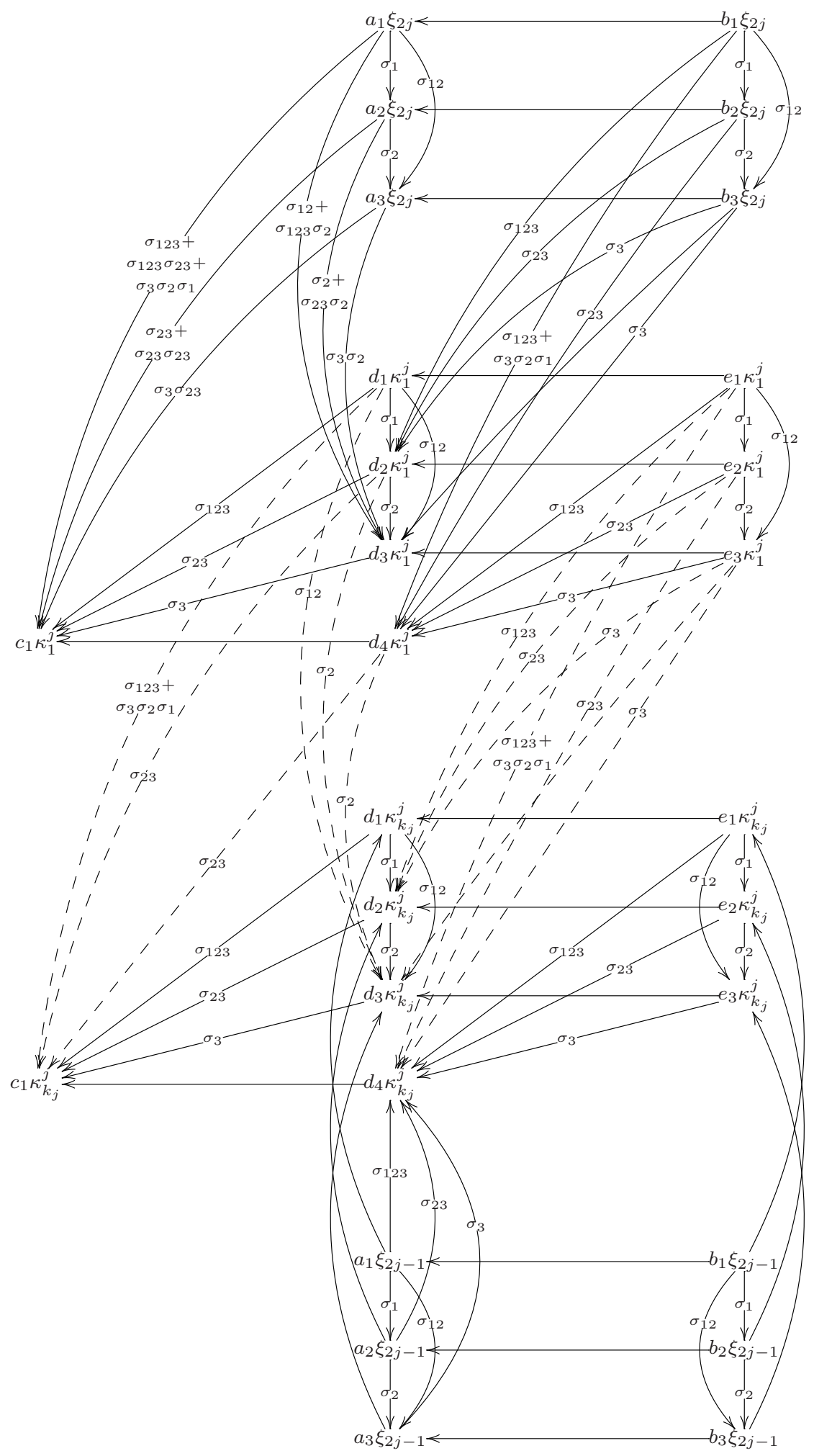

Figure 15. The subspace $P_{\text {vert }}^{j}$, corresponding to a vertical stable chain $\xi_{2 j} \stackrel{\rho_{123}}{\longrightarrow} \kappa_{1}^{j} \stackrel{\rho_{23}}{\longrightarrow} \cdots \stackrel{\rho_{23}}{\longrightarrow} \kappa_{k_{j}}^{j} \stackrel{\rho_{1}}{\longleftarrow} \xi_{2 j-1}$. 


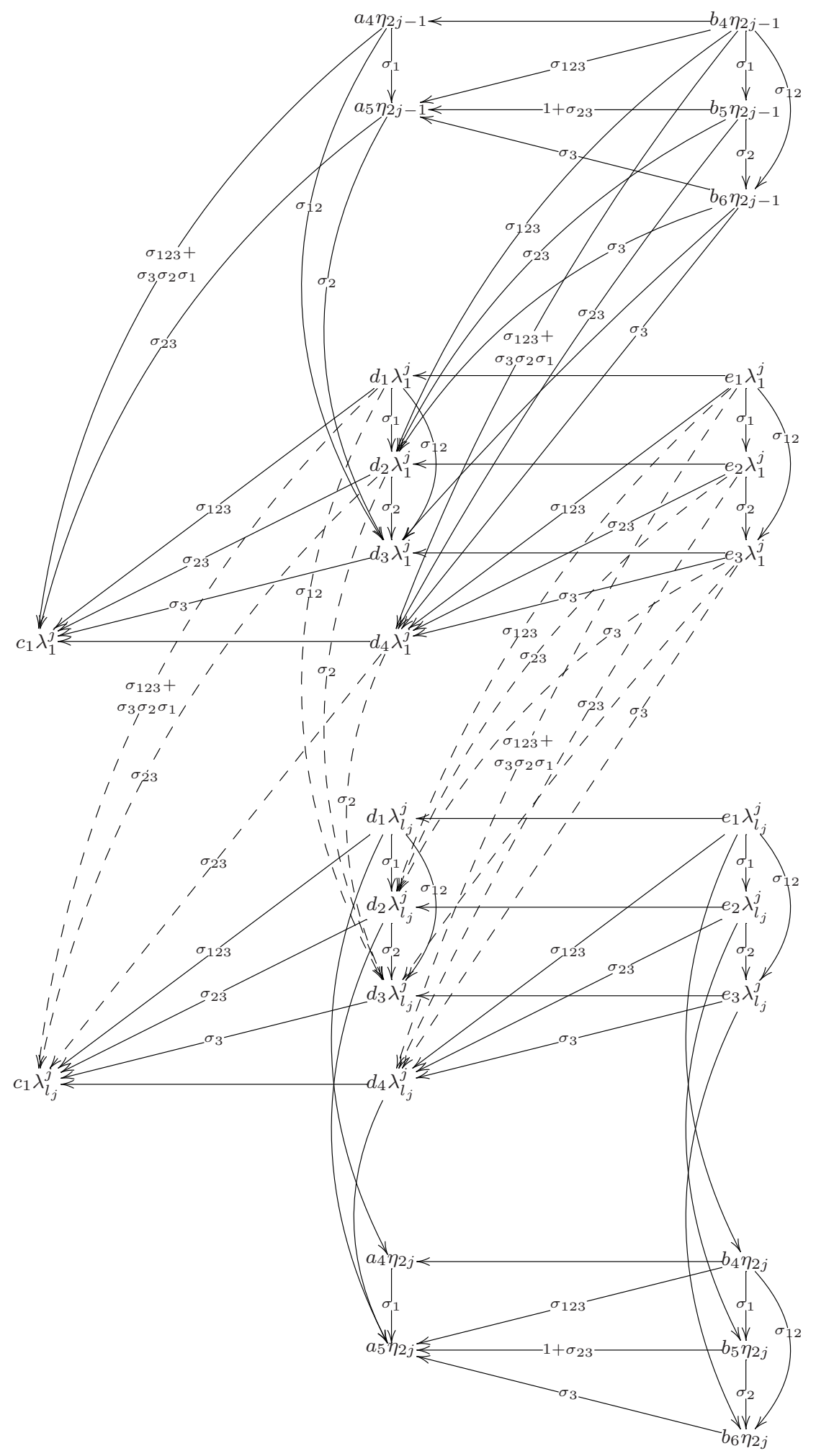

FiguRE 16. The subspace $P_{\text {hor }}^{j}$, corresponding to a horizontal stable chain $\eta_{2 j-1} \stackrel{\rho_{3}}{\longrightarrow} \lambda_{1}^{j} \stackrel{\rho_{23}}{\longrightarrow} \cdots \stackrel{\rho_{23}}{\longrightarrow} \lambda_{l_{j}}^{j} \stackrel{\rho_{2}}{\longrightarrow} \eta_{2 j}$. 


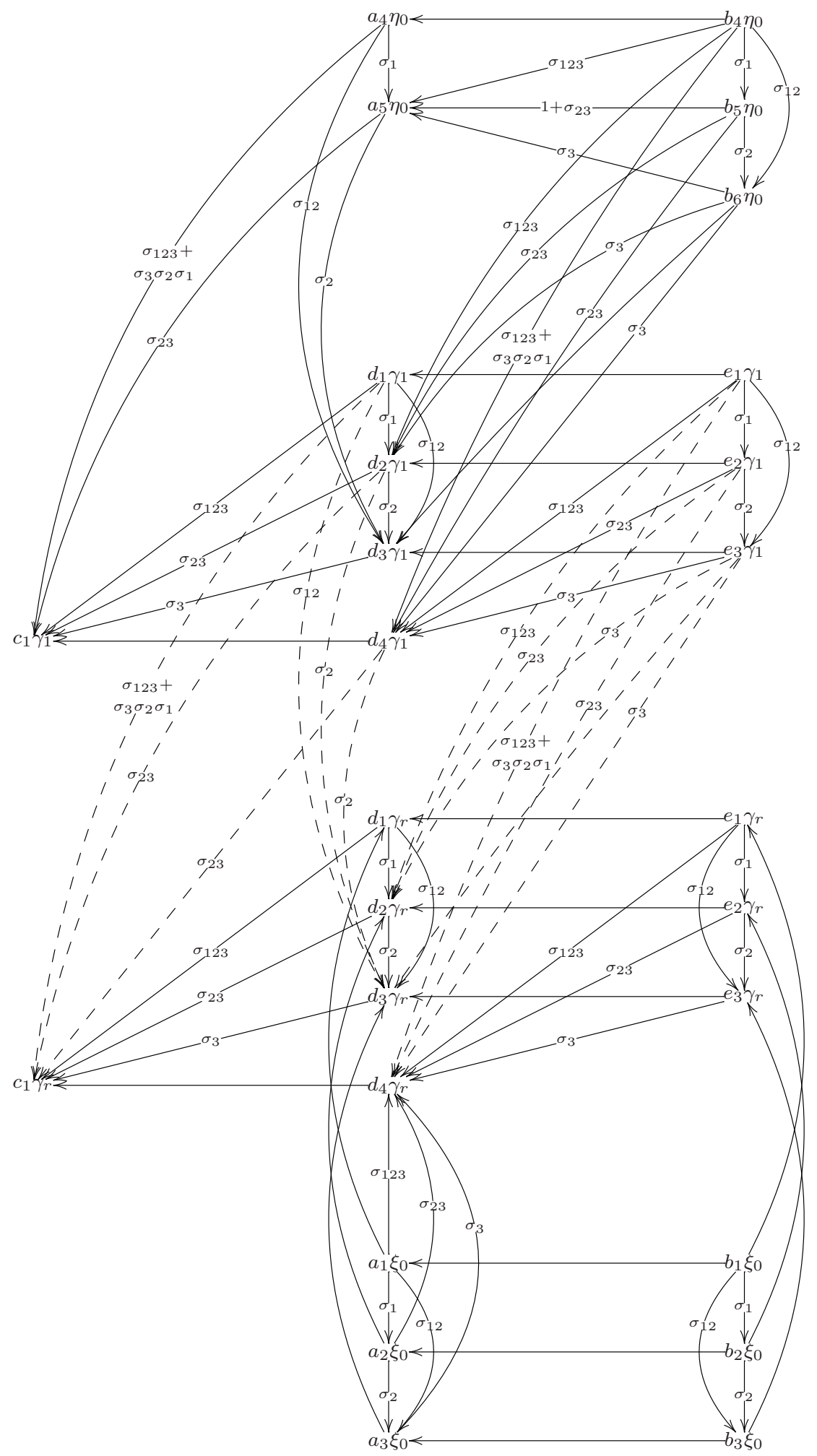

Figure 17. The subspace $P_{\text {unst }}$ when $s<2 \tau(J)$, corresponding to the unstable chain $\eta_{0} \stackrel{\rho_{3}}{\longrightarrow} \gamma_{1} \stackrel{\rho_{23}}{\longrightarrow} \cdots \stackrel{\rho_{23}}{\longrightarrow} \gamma_{s} \stackrel{\rho_{1}}{\longleftarrow} \xi_{0}$. 


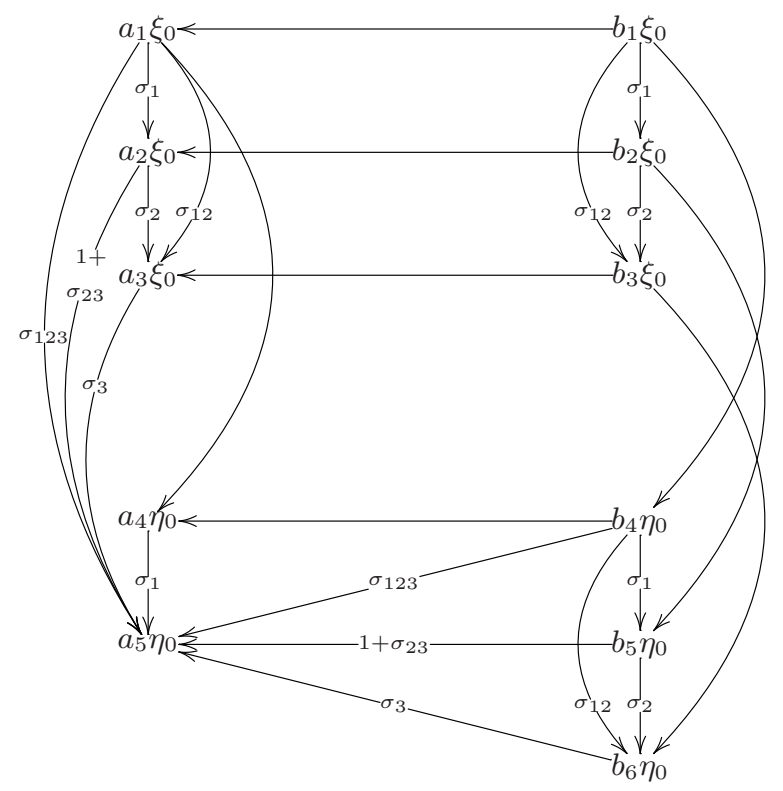

Figure 18. The subspace $P_{\text {unst }}$ when $s=2 \tau(J)$, corresponding to the unstable chain $\xi_{0} \stackrel{\rho_{12}}{\longrightarrow} \eta_{0}$.

- In $P_{\text {vert }}^{j}$, when $k_{j}>1$, there are multiplications

$$
\begin{array}{ll}
b_{1} \xi_{2 j} \stackrel{\sigma_{3} \sigma_{2} \sigma_{12}}{\longrightarrow} d_{3} \kappa_{2}^{j} & b_{1} \xi_{2 j} \stackrel{\sigma_{3} \sigma_{2} \sigma_{123}}{\longrightarrow} c_{1} \kappa_{2}^{j} \\
e_{1} \kappa_{i}^{j} \stackrel{\sigma_{3} \sigma_{2} \sigma_{12}}{\longrightarrow} d_{3} \kappa_{i+2}^{j} & e_{1} \kappa_{i}^{j} \stackrel{\sigma_{3} \sigma_{2} \sigma_{123}}{\longrightarrow} c_{1} \kappa_{i+2}^{j} \quad\left(i=1, \ldots, k_{j}-2\right) .
\end{array}
$$

- In $P_{\text {hor }}^{j}$, when $l_{j}=1$, there is a multiplication $b_{4} \eta_{2 j-1} \stackrel{\sigma_{3} \sigma_{2}}{\longrightarrow} a_{4} \eta_{2 j}$. When $l_{j}>1$, there are multiplications

$$
\begin{aligned}
b_{4} \eta_{2 j-1} \stackrel{\sigma_{3} \sigma_{2} \sigma_{12}}{\longrightarrow} d_{3} \lambda_{2}^{j} & b_{4} \eta_{2 j-1} \stackrel{\sigma_{3} \sigma_{2} \sigma_{123}}{\longrightarrow} c_{1} \lambda_{2}^{j} \\
e_{1} \lambda_{l_{j}-1}^{j} \stackrel{\sigma_{3} \sigma_{2}}{\longrightarrow} a_{4} \eta_{2 j} & \\
e_{1} \lambda_{i}^{j} \stackrel{\sigma_{3} \sigma_{2} \sigma_{12}}{\longrightarrow} d_{3} \lambda_{i+2}^{j} & e_{1} \lambda_{i}^{j} \stackrel{\sigma_{3} \sigma_{2} \sigma_{123}}{\longrightarrow} c_{1} \lambda_{i+2}^{j} \quad\left(i=1, \ldots, l_{j}-2\right) .
\end{aligned}
$$

- In $P_{\text {unst }}$ in the case when $s<2 \tau(J)-1$, there are multiplications

$$
\begin{array}{ll}
b_{4} \eta_{0} \stackrel{\sigma_{3} \sigma_{2} \sigma_{12}}{\longrightarrow} d_{3} \gamma_{2} & b_{4} \eta_{0} \stackrel{\sigma_{3} \sigma_{2} \sigma_{123}}{\longrightarrow} c_{1} \gamma_{2} \\
e_{1} \gamma_{i} \stackrel{\sigma_{3} \sigma_{2} \sigma_{12}}{\longrightarrow} d_{3} \gamma_{i+2} & e_{1} \gamma_{i} \stackrel{\sigma_{3} \sigma_{2} \sigma_{123}}{\longrightarrow} c_{1} \gamma_{i+2} \quad(i=1, \ldots, r-2) .
\end{array}
$$

Finally, we must consider the multiplications in the tensor product that do not respect the splitting in (4.2). These arise from sequences of arrows in $\widehat{\operatorname{CFD}}\left(\mathcal{X}_{J}^{s}\right)$ that involve multiple stable or unstable chains, and they depend on the change-of-basis coefficients relating $\left\{\eta_{0}, \ldots, \eta_{2 n}\right\}$ and $\left\{\xi_{0}, \ldots, \xi_{2 n}\right\}$. 
For instance, if $\eta_{2 j}=\xi_{2 h}$ (where $j, h \in\{1, \ldots, n\}$ ), then $\widehat{\operatorname{CFD}}\left(\mathcal{X}_{J}^{s}\right)$ contains a string of arrows of the form

$$
\eta_{2 j-1} \stackrel{\rho_{3}}{\longrightarrow} \lambda_{1}^{j} \stackrel{\rho_{23}}{\longrightarrow} \cdots \stackrel{\rho_{23}}{\longrightarrow} \lambda_{l_{j}}^{j} \stackrel{\rho_{2}}{\longrightarrow} \eta_{2 j} \stackrel{\rho_{123}}{\longrightarrow} \kappa_{1}^{h} \stackrel{\rho_{23}}{\longrightarrow} \cdots \stackrel{\rho_{23}}{\longrightarrow} \kappa_{k_{h}}^{h} .
$$

Any multiplication in $\widehat{\mathrm{CFAA}}\left(\mathcal{Y}, B_{3}, 0\right)$ that uses a contiguous subsequence of

$$
\rho_{3}, \underbrace{\rho_{23}, \ldots, \rho_{23}}_{l_{j}-1 \text { times }}, \rho_{2}, \rho_{123}, \underbrace{\rho_{23}, \ldots, \rho_{23}}_{k_{h}-1 \text { times }}
$$

contributes a nonzero multiplication in the tensor product that need not respect the splitting. Similarly, if $\eta_{2 j}=\xi_{2 h-1}$, then the same is true for contiguous subsequences of

$$
\rho_{3}, \underbrace{\rho_{23}, \ldots, \rho_{23}}_{l_{j}-1 \text { times }}, \rho_{2}, \rho_{1} .
$$

Similar sequences may also occur near the unstable chain, where we take $\xi_{0}$ instead of $\xi_{2 h-1}$ or $\xi_{2 h}$. By Proposition 2.4, these are the only such sequences that occur. More generally, if the coefficient of $\xi_{p}$ in $\eta_{2 j}$ is nonzero, we obtain multiplications that do not respect the splitting in (4.2). We make this notion more precise below.

By inspecting the matrices $M_{x y}$, we see that the only sequences of this form that actually occur in $\widehat{\mathrm{CFAA}}\left(\mathcal{Y}, B_{3}, 0\right)$ are $\rho_{3} \rho_{2} \rho_{123}, \rho_{3} \rho_{2} \rho_{12}$, and $\rho_{3} \rho_{2} \rho_{1}$, which occur in the first three rows of $M_{a c}, M_{a d}, M_{b a}, M_{b c}$, and $M_{b d}$. Accordingly, the only multiplications that do not preserve the splitting arise when there is a horizontal edge $\eta_{2 j-1} \rightarrow \eta_{2 j}$ of length 1 , and they act on the elements $a_{i} \otimes \eta_{2 j-1}$ and $b_{i} \otimes \eta_{2 j-1}(i=1,2,3)$.

Notice that there are no multiplications into or out of any of the subspaces $P_{\text {hor }}^{j}$. Therefore, each $P_{\text {hor }}^{j}$ is actually a direct summand of $\widehat{\mathrm{CFA}}\left(\mathcal{V}, D_{J, s}\right)$ as an $\mathcal{A}_{\infty}$ submodule, as is $P=\bigoplus_{j=1}^{n} P_{\text {vert }}^{j} \oplus P_{\text {unst }}$. This implies that the tensor product $\widehat{\operatorname{CFA}}\left(\mathcal{V}, D_{J, s}\right) \otimes$ $\widehat{\mathrm{CFD}}\left(\mathcal{X}_{K}^{t}\right)$ (whose total homology, ignoring the filtration, is $\widehat{\mathrm{HF}}\left(S^{3}\right) \cong \mathbb{F}$ ) will also split as a direct sum. We shall see in Subsection 4.3 that the direct summand coming from $P$ has odd rank, which implies that it must have a nonzero contribution to the total homology. Therefore, each summand coming from $P_{\text {hor }}^{j}$ is acyclic and hence does not affect the computation of $\tau\left(D_{J, s}(K, t)\right)$. Thus, we shall henceforth ignore the submodules $P_{\text {hor }}^{j}$.

It is preferable to describe all of the multiplications that do not respect the splitting in terms of the bases specified in (4.1). Recall that $\left(x_{p, q}\right)$ and $\left(y_{p, q}\right)$ are the changeof-basis matrices, so that $\xi_{p}=\sum_{q=0}^{2 n} x_{p, q} \eta_{q}$ and $\eta_{p}=\sum_{q=0}^{2 n} y_{p, q} \xi_{q}$. Let $\mathfrak{j}$ denote the set $\left\{j \in\{1, \ldots, n\} \mid l_{j}=1\right\}$. For each $p \in\{0, \ldots, 2 n\}$ and $h \in\{1, \ldots, n\}$, each $j \in \mathfrak{j}$ for which $x_{p, 2 j-1}=1$ and $y_{2 j, 2 h-1}=1$ contributes multiplications (which we will specify shortly) from $a_{i} \xi_{p}$ and/or $b_{i} \xi_{p}(i=1,2,3)$ into $P_{\text {vert }}^{h}$ via the sequence $\rho_{3} \rho_{2} \rho_{123}$. Of course, multiple values of $j$ may satisfy this criterion, but they all contribute the same multiplications, so we really only care about the count of such $j$ modulo 2 . That is, define $u_{p, h}=\sum_{j \in \mathrm{j}} x_{p, 2 j-1} y_{2 j, 2 h-1}$; there are multiplications from $a_{i} \xi_{p}$ and $b_{i} \xi_{p}$ into $P_{\text {vert }}^{h}$ iff $u_{p, h}=1$. 


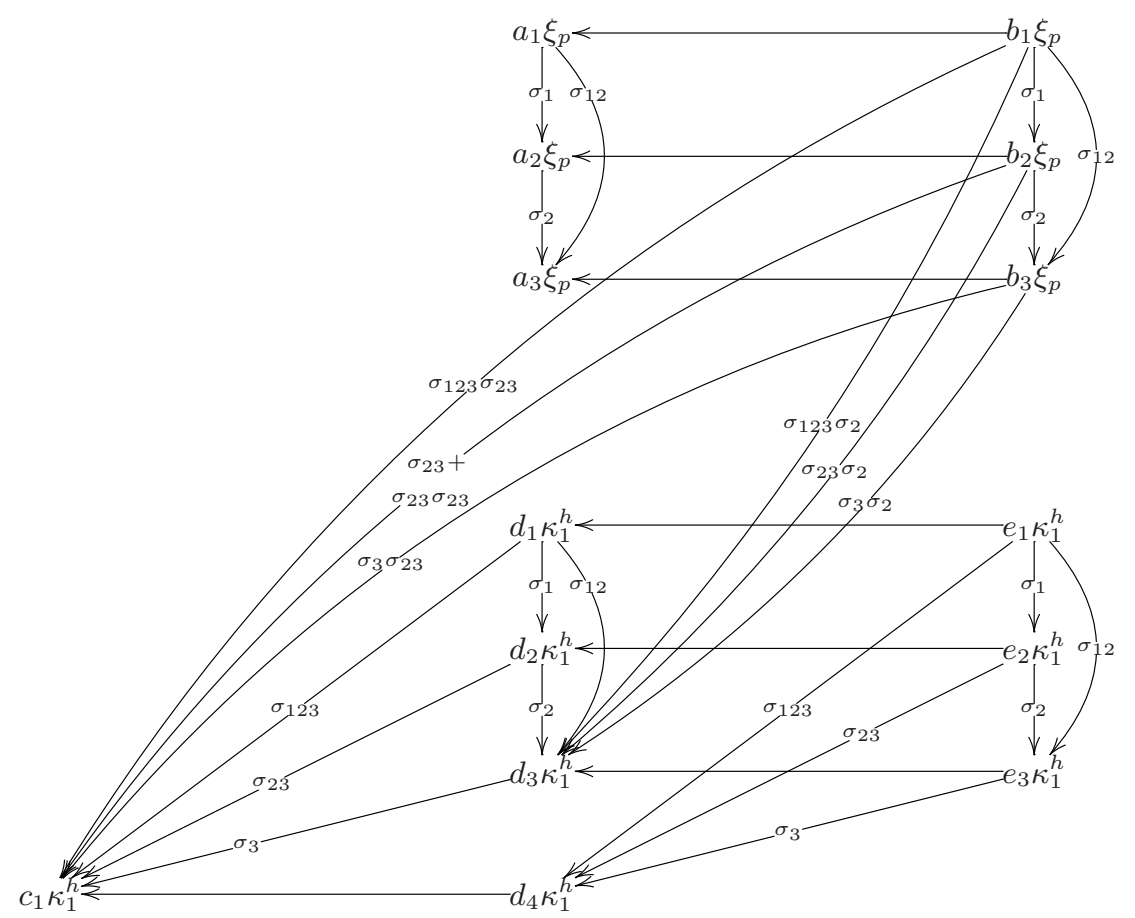

FigurE 19. Multiplications coming from a sequence $\rho_{3} \rho_{2} \rho_{123}$ when $u_{p, h}=1$.

Similarly, each $j$ for which $x_{p, 2 j-1}=1$ and $y_{2 j, 2 h}(h=1, \ldots, n)$ contributes multiplications via $\rho_{3} \rho_{2} \rho_{1}$, so define $v_{p, h}=\sum_{j \in \mathrm{j}} x_{p, 2 j-1} y_{2 j, 2 h}$. Finally, we set $w_{p}=$ $\sum_{j \in \mathrm{j}} x_{p, 2 j-1} y_{2 h, 0}$; this determines whether there are additional multiplications from $a_{i} \xi_{p}$ and $b_{i} \xi_{p}$ into the unstable chain via $\rho_{3} \rho_{2} \rho_{1}, \rho_{3} \rho_{2} \rho_{12}$, or $\rho_{3} \rho_{2} \rho_{123}$, according to whether $s<2 \tau(J), s=2 \tau(J)$ or $s>2 \tau(K)$, respectively (although we are ignoring the third case).

We now specify these multiplications:

- If $u_{p, h}=1$, the sequence $\rho_{3} \rho_{2} \rho_{123}$ provides the multiplications shown in Figure 19.

- If $v_{p, h}=1$, the sequence $\rho_{3} \rho_{2} \rho_{1}$ provides the multiplications shown in Figure 20.

- If $s<2 \tau(J)$ and $w_{p}=1$, the sequence $\rho_{3} \rho_{2} \rho_{1}$ provides the multiplications shown in Figure 20, where we replace $\kappa_{k_{h}}^{h}$ by $\gamma_{r}$. 


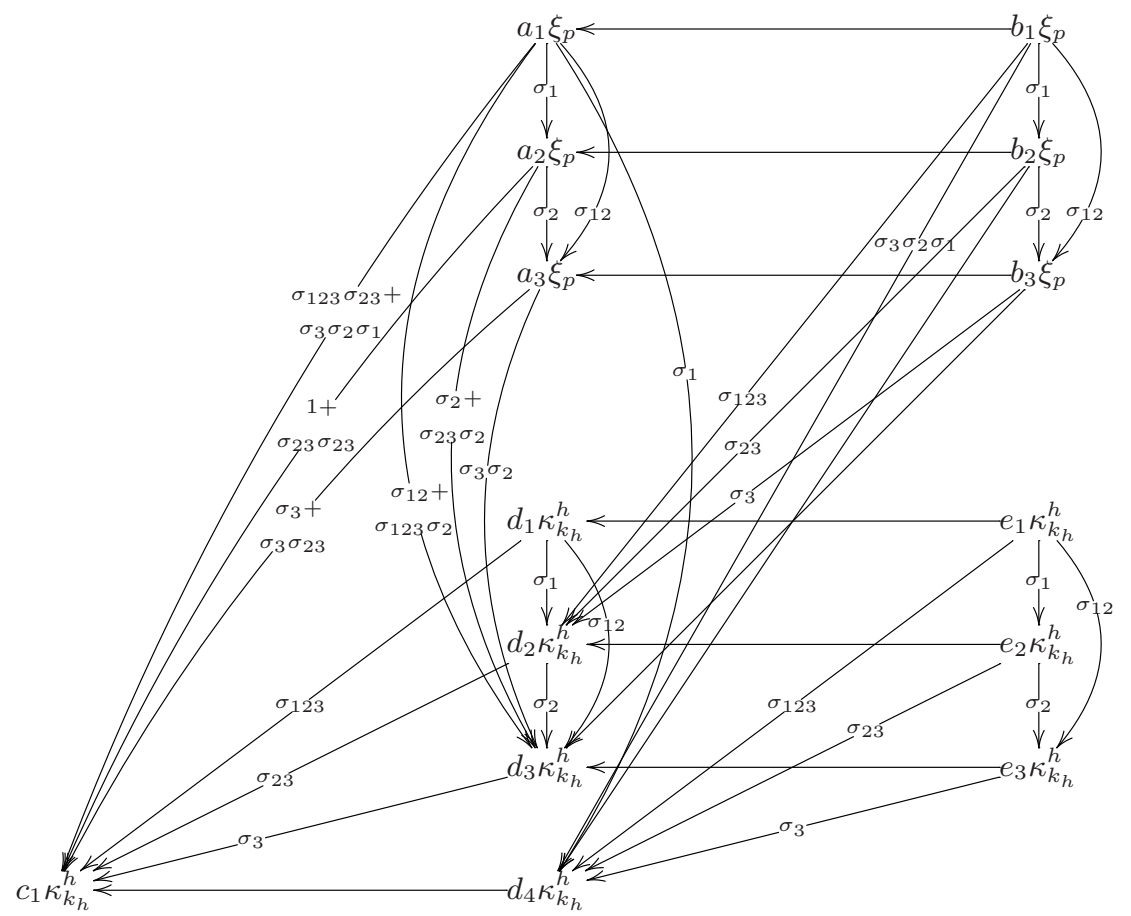

FigurE 20. Multiplications coming from a sequence $\rho_{3} \rho_{2} \rho_{1}$ when $v_{p, h}=1$. If $w_{p}=1$ and $s<2 \tau(K)$, we obtain the same multiplications by replacing $\kappa_{k_{h}}^{h}$ by $\gamma_{r}$.

- Finally, if $s=2 \tau(K)$ and $w_{p}=1$, the sequence $\rho_{3} \rho_{2} \rho_{12}$ provides the following multiplications:

$$
\begin{aligned}
& a_{1} \xi_{p} \stackrel{\sigma_{1}}{\longrightarrow} a_{5} \eta_{0} \\
& b_{1} \xi_{p} \stackrel{\sigma_{123}}{\longrightarrow} a_{5} \eta_{0} \\
& b_{2} \xi_{p} \stackrel{1+\sigma_{23}}{\longrightarrow} a_{5} \eta_{0} \\
& b_{3} \xi_{p} \stackrel{\sigma_{3}}{\longrightarrow} a_{5} \eta_{0} .
\end{aligned}
$$

4.2. Simplification of $\widehat{\mathrm{CFA}}\left(\mathcal{V}, D_{J, s}\right)$. Next, we may simplify $\widehat{\mathrm{CFA}}\left(\mathcal{V}, D_{J, s}\right)$ by canceling unmarked edges that preserve the filtration level. In order to keep track of additional edges that may appear, we must look carefully at the order of cancellation. As mentioned above, we ignore the direct summands $P_{\text {hor }}^{j}$. Define $P^{0}=P_{\text {unst }}$ and $P^{j}=P_{\text {vert }}^{j}$.

Assume first that $s<2 \tau(J)$.

For each $j \in\{1, \ldots, n\}$, in $P^{j}$, we may cancel the differentials $b_{1} \xi_{2 j-1} \rightarrow e_{1} \kappa_{k_{j}}^{j}$, $b_{2} \xi_{2 j-1} \rightarrow e_{2} \kappa_{k_{j}}^{j}, b_{2} \xi_{2 j-1} \rightarrow e_{2} \kappa_{k_{j}}^{j}$, and $a_{1} \xi_{2 j-1} \rightarrow d_{1} \kappa_{k_{j}}^{j}$. Since the targets of those arrows do not lie at the heads of any other arrows, no additional arrows are introduced. Similarly, in $P^{0}$, cancel $b_{1} \xi_{0} \rightarrow e_{1} \gamma_{r}, b_{2} \xi_{0} \rightarrow e_{2} \gamma_{r}, b_{2} \xi_{0} \rightarrow e_{2} \gamma_{r}$, and $a_{1} \xi_{0} \rightarrow d_{1} \gamma_{r}$. 
Next, we cancel the differentials $a_{2} \xi_{2 j-1} \rightarrow d_{2} \kappa_{k_{j}}^{j}$ and $a_{2} \xi_{0} \rightarrow d_{2} \gamma_{r}$. Because of the edge $a_{2} \xi_{2 j-1} \stackrel{\sigma_{23}}{\longrightarrow} d_{4} \kappa_{k_{j}}^{j}$, canceling $a_{2} \xi_{2 j-1} \rightarrow d_{2} \kappa_{k_{j}}^{j}$ introduces new multiplications:

$$
\begin{array}{ll}
e_{1} \kappa_{k_{j}-1}^{j} \stackrel{\sigma_{123} \sigma_{2}}{\longrightarrow} a_{3} \xi_{2 j-1} & e_{1} \kappa_{k_{j}-1}^{j} \stackrel{\sigma_{123} \sigma_{23}}{\longrightarrow} d_{4} \kappa_{k_{j}}^{j} \\
e_{2} \kappa_{k_{j}-1}^{j} \stackrel{\sigma_{23} \sigma_{2}}{\longrightarrow} a_{3} \xi_{2 j-1} & e_{2} \kappa_{k_{j}-1}^{j} \stackrel{\sigma_{23} \sigma_{23}}{\longrightarrow} d_{4} \kappa_{k_{j}}^{j} \\
e_{3} \kappa_{k_{j}-1}^{j} \stackrel{\sigma_{3} \sigma_{2}}{\longrightarrow} a_{3} \xi_{2 j-1} & e_{3} \kappa_{k_{j}-1}^{j} \stackrel{\sigma_{3} \sigma_{23}}{\longrightarrow} d_{4} \kappa_{k_{j}}^{j} .
\end{array}
$$

(If $k_{j}=1$, then replace $e_{i} \kappa_{k_{j}-1}^{j}$ by $b_{i} \xi_{2 j}$ in (4.7).) We shall examine the effects of these cancellations on the edges that do not respect the splitting momentarily.

Next, because of the edge $a_{3} \xi_{2 j-1} \stackrel{\sigma_{3}}{\rightarrow} d_{4} \kappa_{k_{j}}^{j}$, canceling $a_{3} \xi_{2 j-1} \rightarrow d_{3} \kappa_{k_{j}}^{j}$ removes the edge $e_{3} \kappa_{k_{j}-1}^{j} \stackrel{\sigma_{3}}{\longrightarrow} d_{4} \kappa_{k_{j}}^{j}$ and adds edges

$$
\begin{aligned}
& d_{1} \kappa_{k_{j}-1}^{j} \stackrel{\sigma_{12} \sigma_{3}}{\longrightarrow} d_{4} \kappa_{k_{j}}^{j} \\
& d_{2} \kappa_{k_{j}-1}^{j} \stackrel{\sigma_{2} \sigma_{3}}{\longrightarrow} d_{4} \kappa_{k_{j}}^{j} \\
& d_{4} \kappa_{k_{j}-1}^{j} \stackrel{\sigma_{2} \sigma_{3}}{\longrightarrow} d_{4} \kappa_{k_{j}}^{j} \\
& e_{1} \kappa_{k_{j}-2}^{j} \stackrel{\sigma_{3} \sigma_{2} \sigma_{12} \sigma_{3}}{\longrightarrow} d_{4} \kappa_{k_{j}}^{j} .
\end{aligned}
$$

Because we will ultimately tensor with $\widehat{\mathrm{CFD}}\left(X_{K}^{t}\right)$, in which the sequences $\sigma_{2} \sigma_{3}$ and $\sigma_{12} \sigma_{3}$ do not appear, we may disregard these four edges. We also eliminate the edge $e_{3} \kappa_{k_{j}-1}^{j} \stackrel{\sigma_{3}}{\rightarrow} d_{4} \kappa_{k_{j}-1}^{j}$. The same thing occurs in $P^{0}$ when we cancel $a_{3} \xi_{0} \rightarrow d_{3} \gamma_{r}$.

Let $Q^{j}$ denote the module resulting from $P^{j}$ after the cancellations just described. The multiplications on $Q^{j}$ are shown in Figures 21 and 22 and equations (4.3) and (4.5).

Now we keep track of what these cancellations do to the edges that do not respect the splitting, as shown in Figures 19 and 20.

If $u_{p, j}=1$, then there are edges from $b_{i} \xi_{p}$ to $d_{3} \kappa_{1}^{j}$, as shown in Figure 19. If $k_{j}=1$, then canceling $a_{3} \xi_{2 j-1} \rightarrow d_{3} \kappa_{1}^{j}$ will introduce new multiplications coming from $b_{i} \xi_{p}$, but all of these multiplications involve $\sigma_{2} \sigma_{3}$ or $\sigma_{12} \sigma_{3}$ and may thus be disregarded. Also, when $p=2 m+1$ or $p=0$ these edges are eliminated when we cancel $b_{i} \xi_{2 m+1} \rightarrow e_{i} \kappa_{k_{m}}^{m}$ or $b_{i} \xi_{0} \rightarrow e_{i} \gamma_{r}$, respectively.

If $v_{p, j}=1$, when we cancel $a_{2} \xi_{2 j-1} \rightarrow d_{2} \kappa_{k_{j}}^{j}$, we obtain multiplications

$$
\begin{array}{ll}
b_{1} \xi_{p} \stackrel{\sigma_{123} \sigma_{2}}{\longrightarrow} a_{3} \xi_{2 j-1} & b_{1} \xi_{p} \stackrel{\sigma_{123} \sigma_{23}}{\longrightarrow} d_{4} \kappa_{k_{j}}^{j} \\
b_{2} \xi_{p} \stackrel{\sigma_{23} \sigma_{2}}{\longrightarrow} a_{3} \xi_{2 j-1} & b_{2} \xi_{p} \stackrel{\sigma_{23} \sigma_{23}}{\longrightarrow} d_{4} \kappa_{k_{j}}^{j} \\
b_{3} \xi_{p} \stackrel{\sigma_{3} \sigma_{23}}{\longrightarrow} d_{4} \kappa_{k_{j}}^{j} & b_{3} \xi_{p} \stackrel{\sigma_{3} \sigma_{2}}{\longrightarrow} a_{3} \xi_{2 j-1}
\end{array}
$$




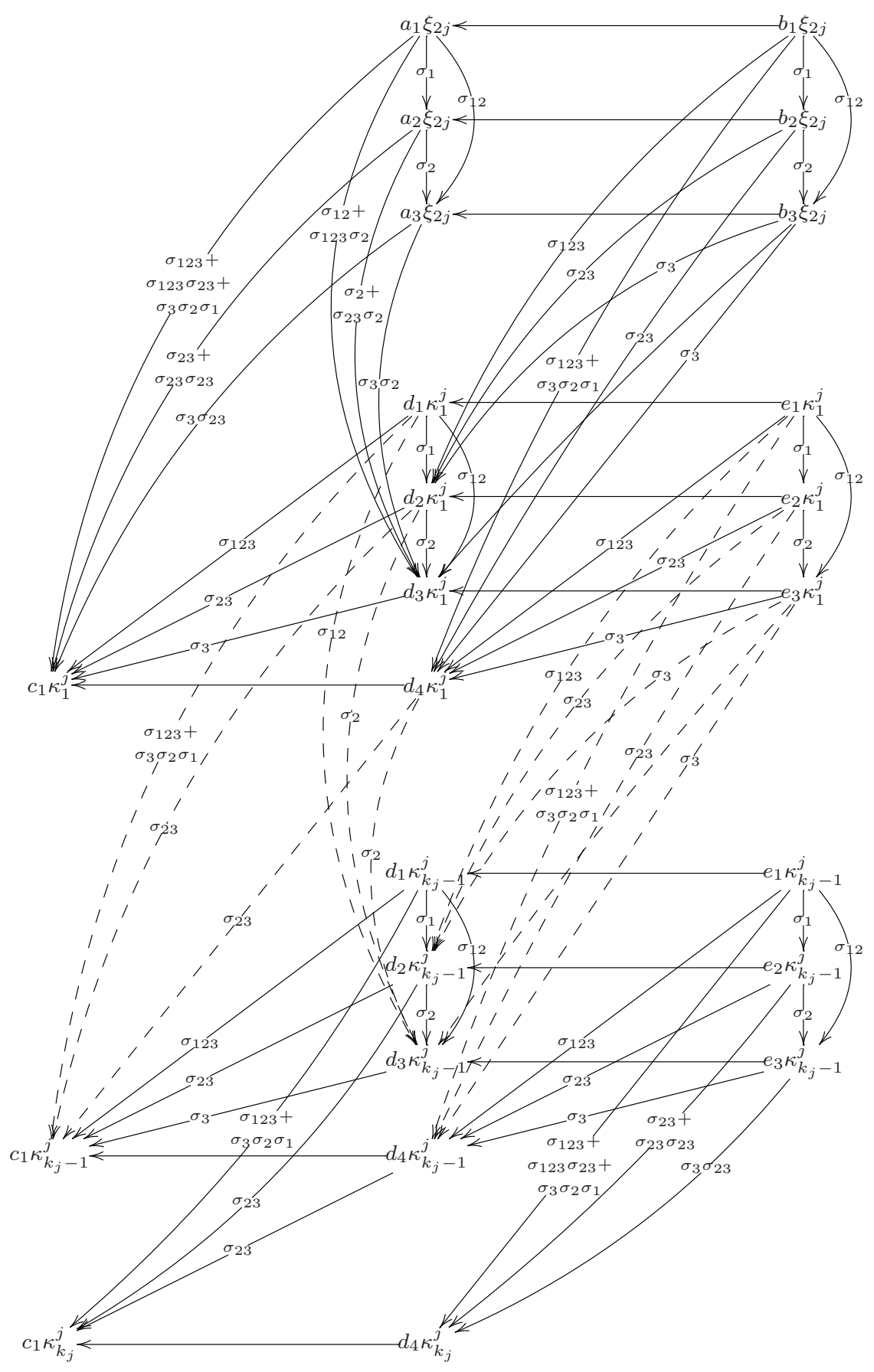

Figure 21. The subspace $Q^{j}(j>0)$ obtained from $P_{\text {vert }}^{j}$ by canceling edges.

in addition to the ones already appearing in Figure 20. When we then cancel $a_{3} \xi_{2 j-1} \rightarrow d_{3} \kappa_{k_{j}}^{j}$, we obtain new multiplications:

$$
\begin{aligned}
& a_{1} \xi_{p} \stackrel{\sigma_{12} \sigma_{3}+\sigma_{123} \sigma_{2} \sigma_{3}}{\longrightarrow} d_{4} \kappa_{k_{j}}^{j} \\
& a_{2} \xi_{p} \stackrel{\sigma_{2} \sigma_{3}+\sigma_{23} \sigma_{2} \sigma_{3}}{\longrightarrow} d_{4} \kappa_{k_{j}}^{j} \\
& a_{3} \xi_{p} \stackrel{\sigma_{3} \sigma_{2} \sigma_{3}}{\longrightarrow} d_{4} \kappa_{k_{j}}^{j} \\
& b_{3} \xi_{p} \stackrel{\sigma_{3}}{\longrightarrow} d_{4} \kappa_{k_{j}}^{j}
\end{aligned}
$$




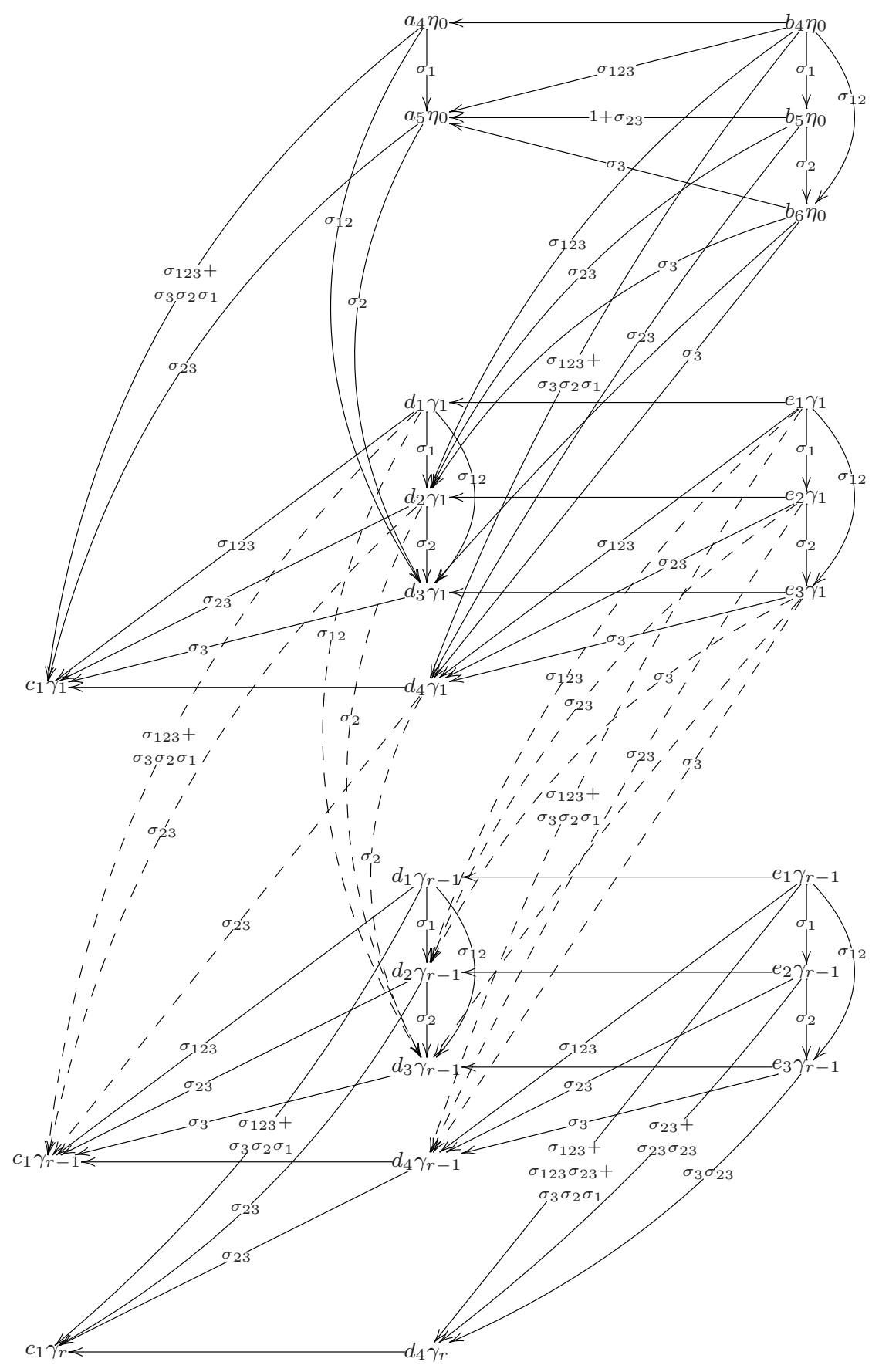

Figure 22. The subspace $Q^{0}$ obtained from $P_{\text {unst }}$ by canceling edges, when $s<2 \tau(J)$. 


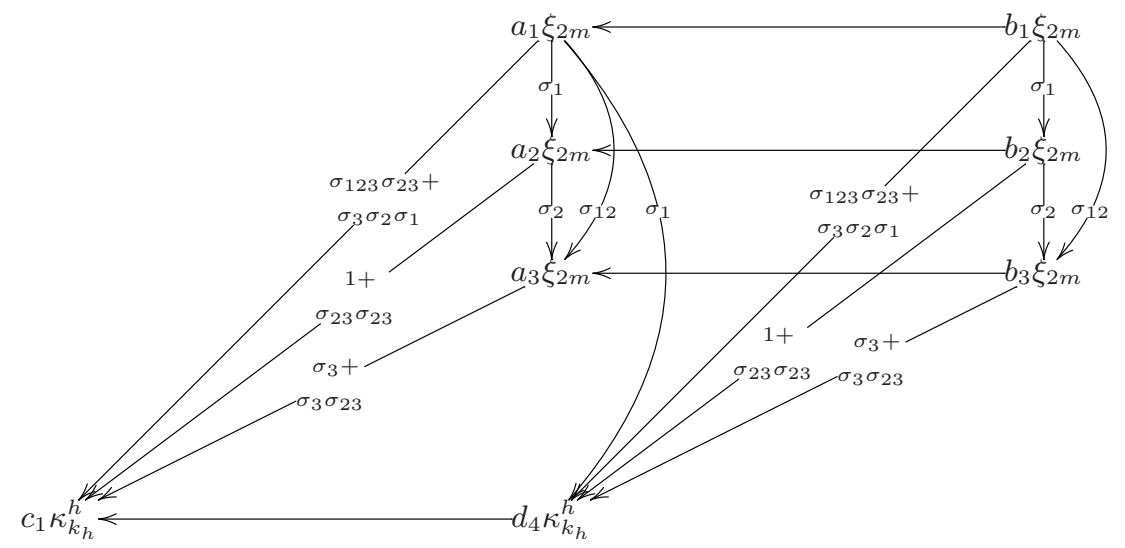

Figure 23. Reduced form of Figure 20 when $p=2 m, m>0$.

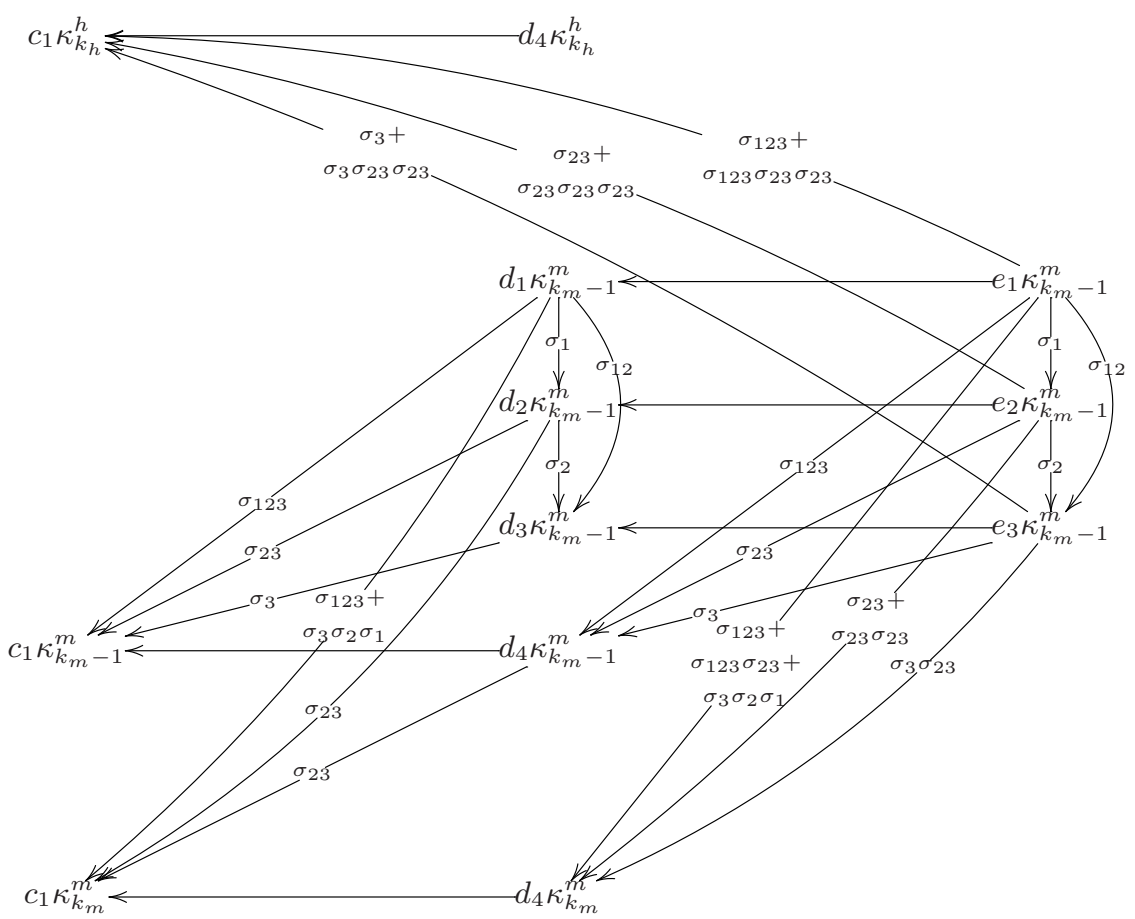

FiguRE 24. Reduced form of Figure 20 in the case where $p=2 m+1$ (or $p=0$, replacing $\kappa_{k_{m}-1}^{m}$ by $\gamma_{r-1}$ and $\kappa_{k_{m}}^{m}$ by $\gamma_{r}$ ).

Most of these may be disregarded by Proposition 2.4. If $p=2 m$ for $m>0$, the resulting reduced form of Figure 20 is shown in Figure 23. On the other hand, if $p=2 m+1$, we also cancel the edges $a_{i} \xi_{2 m+1} \rightarrow d_{i} \kappa_{k_{m}}^{m}$ and $b_{i} \xi_{2 m+1} \rightarrow e_{i} \kappa_{k_{m}}^{m}$, introducing the multiplications shown in Figure 24. Similarly, if $p=0$, we cancel the edges $a_{i} \xi_{0} \rightarrow d_{i} \gamma_{r}$ and $b_{i} \xi_{0} \rightarrow e_{i} \gamma_{r}$, introducing similar multiplications. 
We now return to the case where $s=2 \tau(J)$. In $P_{\text {unst }}$, the edges $a_{1} \xi_{0} \rightarrow a_{4} \eta_{0}$, $b_{1} \xi_{0} \rightarrow b_{4} \eta_{0}, b_{2} \xi_{0} \rightarrow b_{5} \eta_{0}$, and $b_{3} \xi_{0} \rightarrow b_{6} \eta_{0}$ cancel, and since their targets do not have any other incoming edges, no new multiplications are introduced. The only three remaining generators are $a_{2} \xi_{0}, a_{3} \xi_{0}$, and $a_{5} \eta_{0}$, all in filtration level 0 , with the following multiplications:

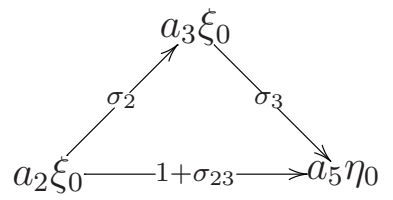

As above, $a_{2} \xi_{0}$ and $a_{3} \xi_{0}$ may have some outgoing edges, and $a_{5} \eta_{0}$ may have some incoming ones. The rest of the argument goes through unchanged.

4.3. Tensor product over $\mathcal{A}_{\sigma}$. Let $Q=\bigoplus_{j=0}^{n} Q^{j}$, with multiplications as described

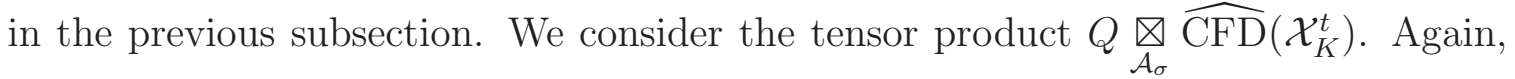
the goal is to obtain a decomposition of the tensor product according to the stable and unstable chains in $\widehat{\mathrm{CFD}}\left(\mathcal{X}_{K}^{t}\right)$.

It is convenient to give the generators of $Q^{j}$ new names. For $j=1, \ldots, n$ and $i=1, \ldots, k_{j}-1$, define:

$$
\begin{array}{llll}
A^{j}=a_{1} \xi_{2 j} & A^{\prime j}=b_{1} \xi_{2 j} & E_{i}^{j}=d_{1} \kappa_{i}^{j} & E_{i}^{\prime j}=e_{1} \kappa_{i}^{j} \\
B^{j}=a_{2} \xi_{2 j} & B^{\prime j}=b_{2} \xi_{2 j} & F_{i}^{j}=d_{2} \kappa_{i}^{j} & F_{i}^{\prime j}=e_{2} \kappa_{i}^{j} \\
C^{j}=a_{3} \xi_{2 j} & C^{\prime j}=b_{3} \xi_{2 j} & G_{i}^{j}=d_{3} \kappa_{i}^{j} & G_{i}^{\prime j}=e_{3} \kappa_{i}^{j} \\
D^{j}=c_{1} \kappa_{k_{j}}^{j} & D^{\prime j}=d_{4} \kappa_{k_{j}}^{j} & H_{i}^{j}=c_{1} \kappa_{i}^{j} & H_{i}^{\prime j}=d_{4} \kappa_{i}^{j}
\end{array}
$$

When $s<2 \tau(J)$, for $i=1, \ldots, r-1$, define:

$$
\begin{array}{llll}
A^{0}=a_{4} \eta_{0} & A^{\prime 0}=b_{4} \eta_{0} & E_{i}^{0}=d_{1} \gamma_{i} & E_{i}^{\prime 0}=e_{1} \gamma_{i} \\
B^{0}=a_{5} \eta_{0} & B^{\prime 0}=b_{5} \eta_{0} & F_{i}^{0}=d_{2} \gamma_{i} & F_{i}^{\prime 0}=e_{2} \gamma_{i} \\
& C^{\prime 0}=b_{6} \eta_{0} & G_{i}^{0}=d_{3} \gamma_{i} & G_{i}^{\prime 0}=e_{3} \gamma_{i} \\
D^{0}=c_{1} \gamma_{r} & D^{\prime 0}=d_{4} \gamma_{r} & H_{i}^{0}=c_{1} \gamma_{i} & H_{i}^{\prime 0}=d_{4} \gamma_{i}
\end{array}
$$

Also, for notational convenience, define $k_{0}=r$.

We divide up the generators of the subspaces $Q^{j}$ by Alexander grading and idempotent:

\begin{tabular}{|c|c|c|c|}
\hline & $A=-1$ & $A=0$ & $A=1$ \\
\hline$\iota_{0}^{\sigma}$ & & $A^{j}, C^{j}, E_{i}^{j}, G_{i}^{j}$ & $A^{\prime j}, C^{\prime j}, E_{i}^{\prime j}, G_{i}^{\prime j}$ \\
\hline$\iota_{1}^{\sigma}$ & $D^{j}, H_{i}^{j}$ & $B^{j}, D^{\prime j}, F_{i}^{j}, H_{i}^{\prime j}$ & $B^{\prime j}, F_{i}^{\prime j}$ \\
\hline
\end{tabular}

In Figures 21 and 22, notice that of the generators in idempotent $\iota_{0}, A^{j}, A^{\prime j}, E_{i}^{j}$, and $E_{i}^{\prime j}$ have outgoing edges labeled $\sigma_{1}, \sigma_{12}$, and $\sigma_{123}$, while $C^{j}, C^{\prime j}, G_{i}^{j}$, and $G_{i}^{\prime j}$ have outgoing edges labeled $\sigma_{3}$ and incoming edges labeled $\sigma_{2}$ and $\sigma_{12}$. Accordingly, 
it makes sense to associate the former with the vertical chains and the latter with the horizontal chains. That is, for each $J \in\{1, \ldots, N\}$ and $j \in\{0, \ldots, n\}$, define:

$$
\begin{aligned}
Z_{\text {vert }}^{J, j}= & \left\langle A^{j}, A^{\prime j}, E_{i}^{j}, E_{i}^{\prime j}\right\rangle \otimes\left\langle\Xi_{2 J-1}, \Xi_{2 J}\right\rangle \\
& +\left\langle B^{j}, B^{\prime j}, D^{j}, D^{\prime j}, F_{i}^{j}, F_{i}^{\prime j}, H_{i}^{j}, H_{i}^{\prime j}\right\rangle \otimes\left\langle\mathrm{K}_{I}^{J} \mid 1 \leq I \leq K_{J}\right\rangle \\
Z_{\mathrm{hor}}^{J, j}= & \left\langle C^{j}, C^{\prime j}, G_{i}^{j}, G_{i}^{\prime j}\right\rangle \otimes\left\langle\mathrm{H}_{2 J-1}, \mathrm{H}_{2 J}\right\rangle \\
& +\left\langle B^{j}, B^{\prime j}, D^{j}, D^{\prime j}, F_{i}^{j}, F_{i}^{\prime j}, H_{i}^{j}, H_{i}^{\prime j}\right\rangle \otimes\left\langle\Lambda_{I}^{J} \mid 1 \leq I \leq L_{J}\right\rangle \\
Z_{\text {unst }}^{j}= & \left\langle A^{j}, A^{\prime j}, E_{i}^{j}, E_{i}^{\prime j}\right\rangle \otimes\left\langle\Xi_{0}\right\rangle \\
& +\left\langle C^{j}, C^{\prime j}, G_{i}^{j}, G_{i}^{\prime j}\right\rangle \otimes\left\langle\mathrm{H}_{0}\right\rangle \\
& +\left\langle B^{j}, B^{\prime j}, D^{j}, D^{\prime j}, F_{i}^{j}, F_{i}^{\prime j}, H_{i}^{j}, H_{i}^{\prime j}\right\rangle \otimes\left\langle\Gamma_{i} \mid 1 \leq I \leq R\right\rangle .
\end{aligned}
$$

Then, as a vector space,

$$
Q \nabla \widehat{\operatorname{CFD}}\left(\mathcal{X}_{K}^{t}\right)=\bigoplus_{\substack{J=1, \ldots, N \\ j=0, \ldots, n}} Z_{\text {vert }}^{J, j} \oplus \bigoplus_{\substack{J=1, \ldots, N \\ j=0, \ldots, n}} Z_{\text {hor }}^{J, j} \oplus \bigoplus_{j=0}^{n} Z_{\text {unst }}^{j} .
$$

For fixed $J$, we write $Z_{\text {vert }}^{J, *}=\bigoplus_{j=0}^{n} Z_{\text {vert }}^{J, j}$, and so on.

As before, it is easy to verify that the differentials on the tensor product coming from $m_{1}$ and $m_{2}$ multiplications in Figures 21 and 22 respect the splitting (4.13). These differentials are illustrated in Figures 25 through 29. Note that we obtain slightly different differentials depending on whether $j=0$ or $j>0$. The doubledotted arrows correspond to the dashed arrows in Figures 15 through 18, for instance, in Figure 25, the double-dotted arrow from $E_{1}^{\prime j} \Xi_{2 J}$ to $H_{k_{j}-1}^{\prime j} \mathrm{~K}_{1}^{J}$ really means that there are differentials $E_{i}^{\prime j} \Xi_{2 J} \rightarrow H_{i+1}^{\prime j} \mathrm{~K}_{1}^{J}$ for $i=1, \ldots, k_{j}-2$.

Next, we must consider the differentials coming from the remaining multiplications on $Q$. First, we look at differentials that respect the splitting. If $k_{j}>1$, the relevant multiplications on $Q^{j}$ are:

$$
\begin{array}{cccc}
A^{j} \stackrel{\sigma_{123} \sigma_{2}}{\longrightarrow} G_{1}^{j} & B^{j} \stackrel{\sigma_{23} \sigma_{2}}{\longrightarrow} G_{1}^{j} & C^{j} \stackrel{\sigma_{3} \sigma_{2}}{\longrightarrow} G_{1}^{j} & \text { if } j>0 \\
A^{\prime j} \stackrel{\sigma_{123} \sigma_{23}}{\longrightarrow} D^{\prime j} & B^{\prime j} \stackrel{\sigma_{23} \sigma_{23}}{\longrightarrow} D^{\prime j} & C^{\prime j} \stackrel{\sigma_{3} \sigma_{23}}{\longrightarrow} D^{\prime j} & \text { if } k_{j}=1 \\
E_{k_{j}-1}^{\prime j} \stackrel{\sigma_{123} \sigma_{23}}{\longrightarrow} D^{\prime j} & F_{k_{j}-1}^{\prime j} \stackrel{\sigma_{23} \sigma_{23}}{\longrightarrow} D^{\prime j} & G_{k_{j}-1}^{\prime j} \stackrel{\sigma_{3} \sigma_{23}}{\longrightarrow} D^{\prime j} & \text { if } k_{j}>1 .
\end{array}
$$

Therefore:

- In $Z_{\text {vert }}^{J, j}$, if $K_{J}>1$, there are differentials $E_{k_{j-1}}^{\prime j} \Xi_{2 J} \rightarrow D^{\prime j} \mathrm{~K}_{2}^{J}$ and $F_{k_{j-1}}^{\prime j} \mathrm{~K}_{I}^{J} \rightarrow$ $D^{\prime j} \mathrm{~K}_{I+2}^{J}$.

- In $Z_{\text {hor }}^{J, j}$, if $K_{J}>1$, there are differentials $G_{k_{j-1}}^{\prime j} \mathrm{H}_{2 J-1} \rightarrow D^{\prime j} \Lambda_{2}^{J}$ and $F_{k_{j-1}}^{\prime j} \Lambda_{I}^{J} \rightarrow$ $D^{\prime j} \Lambda_{I+2}^{J}$. Additionally, when $j>0$, there are differentials $C^{j} \mathrm{H}_{2 J-1} \rightarrow G_{1}^{j} \mathrm{H}_{2 J}$ if $K_{J}=1$, and $B^{j} \Lambda_{K_{J}-1}^{J} \rightarrow G_{1}^{j} \mathrm{H}_{2 J}$ if $K_{J}>1$.

- In $Z_{\text {unst }}^{j}$, if $t<2 \tau(K)-1$, there are differentials $G_{k_{j-1}}^{\prime j} \mathrm{H}_{0} \rightarrow D^{\prime j} \Gamma_{2}$ and $F_{k_{j-1}}^{\prime j} \Gamma_{I} \rightarrow D^{\prime j} \Gamma_{I+2}$. If $t=2 \tau(K)+1$, there are differentials $A^{j} \Xi_{0} \rightarrow G_{1}^{j} \mathrm{H}_{0}$ 


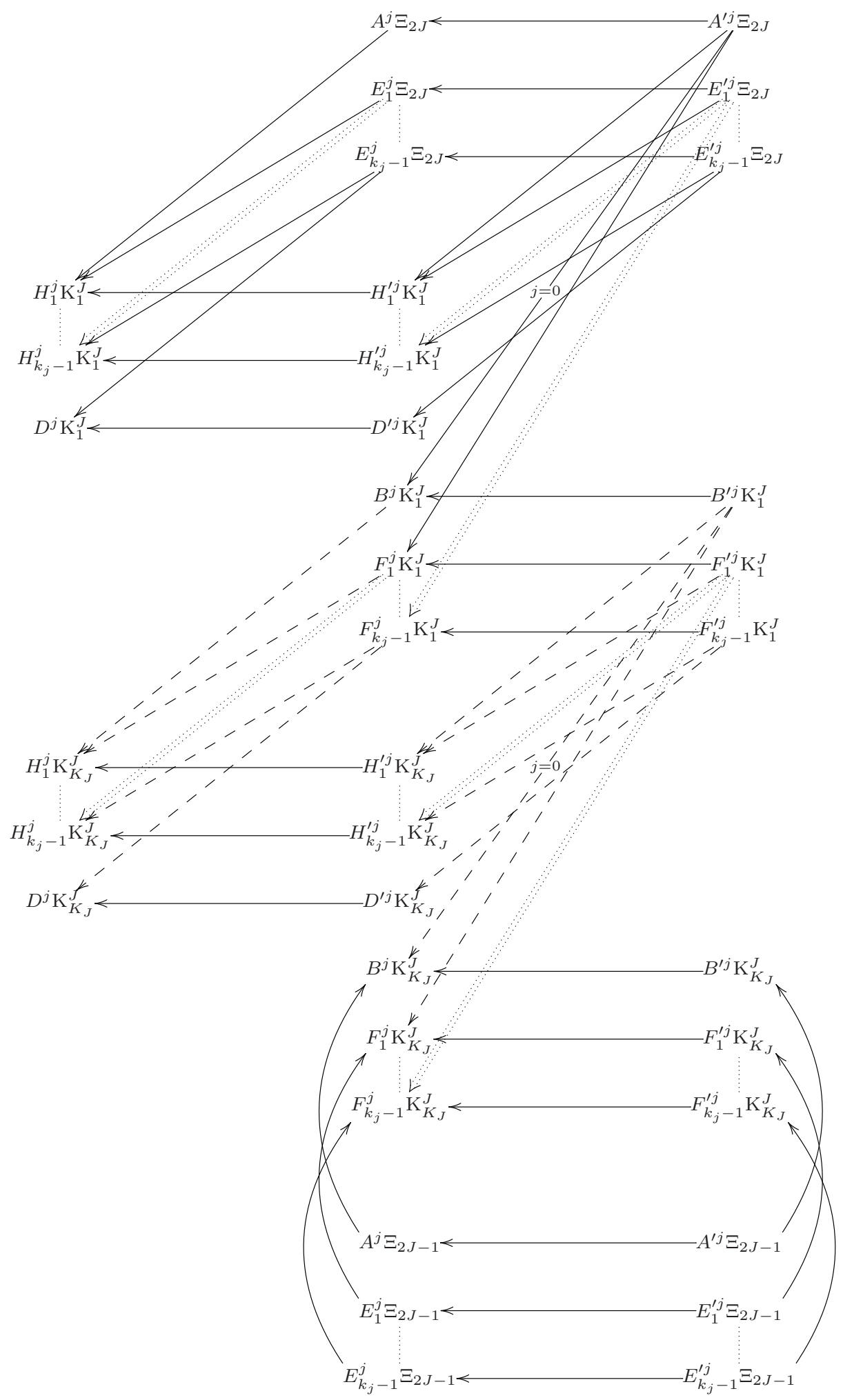

FiguRE 25. The subspace $Z_{\text {vert }}^{J, j}$, corresponding to a vertical stable chain $\Xi_{2 J} \stackrel{\sigma_{123}}{\longrightarrow} \mathrm{K}_{1}^{J} \stackrel{\sigma_{23}}{\longrightarrow} \cdots \stackrel{\sigma_{23}}{\longrightarrow} \mathrm{K}_{K_{J}}^{J} \stackrel{\sigma_{1}}{\longleftarrow} \Xi_{2 J-1}$. 


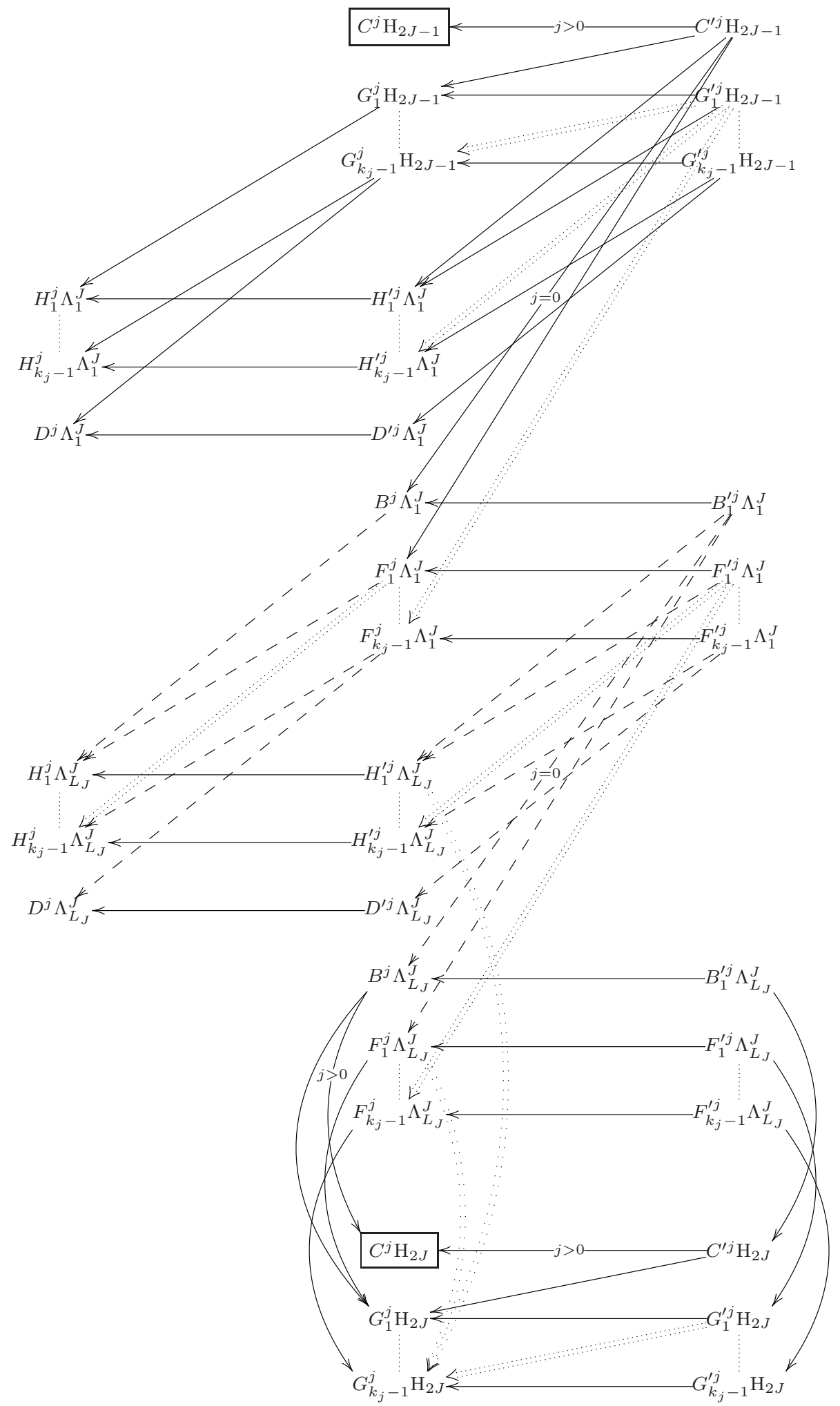

FiguRE 26. The subspace $Z_{\text {hor }}^{J, j}$, corresponding to a horizontal stable chain $\mathrm{H}_{2 J-1} \stackrel{\sigma_{3}}{\longrightarrow} \Lambda_{1}^{J} \stackrel{\sigma_{23}}{\longrightarrow} \cdots \stackrel{\sigma_{23}}{\longrightarrow} \Lambda_{L_{J}}^{J} \stackrel{\sigma_{2}}{\longrightarrow} \mathrm{H}_{2 J}$. 


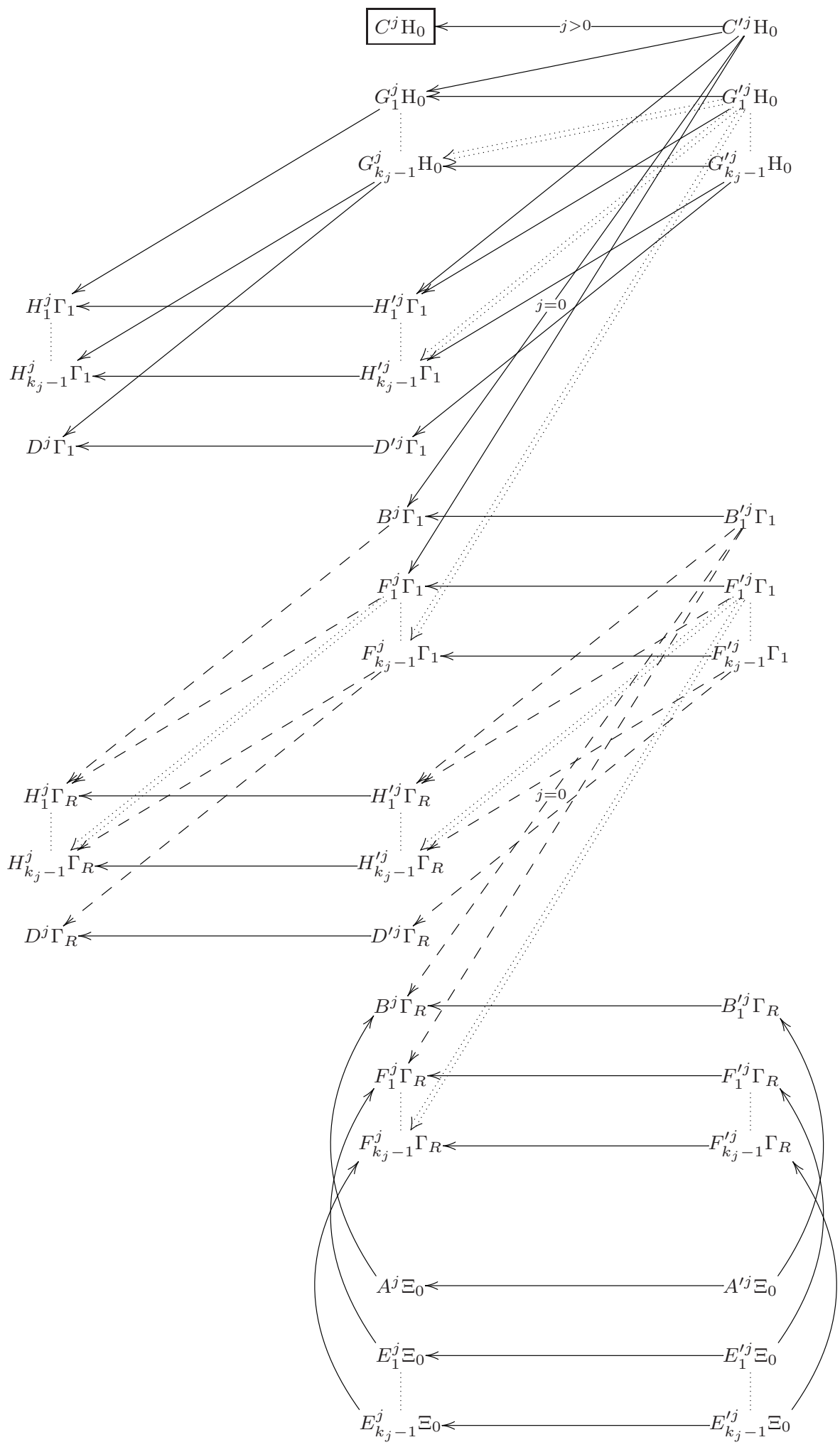

Figure 27. The subspace $Z_{\text {unst }}^{j}$ when $t<2 \tau(K)$, corresponding to the unstable chain $\mathrm{H}_{0} \stackrel{\sigma_{3}}{\longrightarrow} \Gamma_{1} \stackrel{\sigma_{23}}{\longrightarrow} \cdots \stackrel{\sigma_{23}}{\longrightarrow} \Gamma_{R} \stackrel{\sigma_{1}}{\longleftarrow} \Xi_{0}$. 


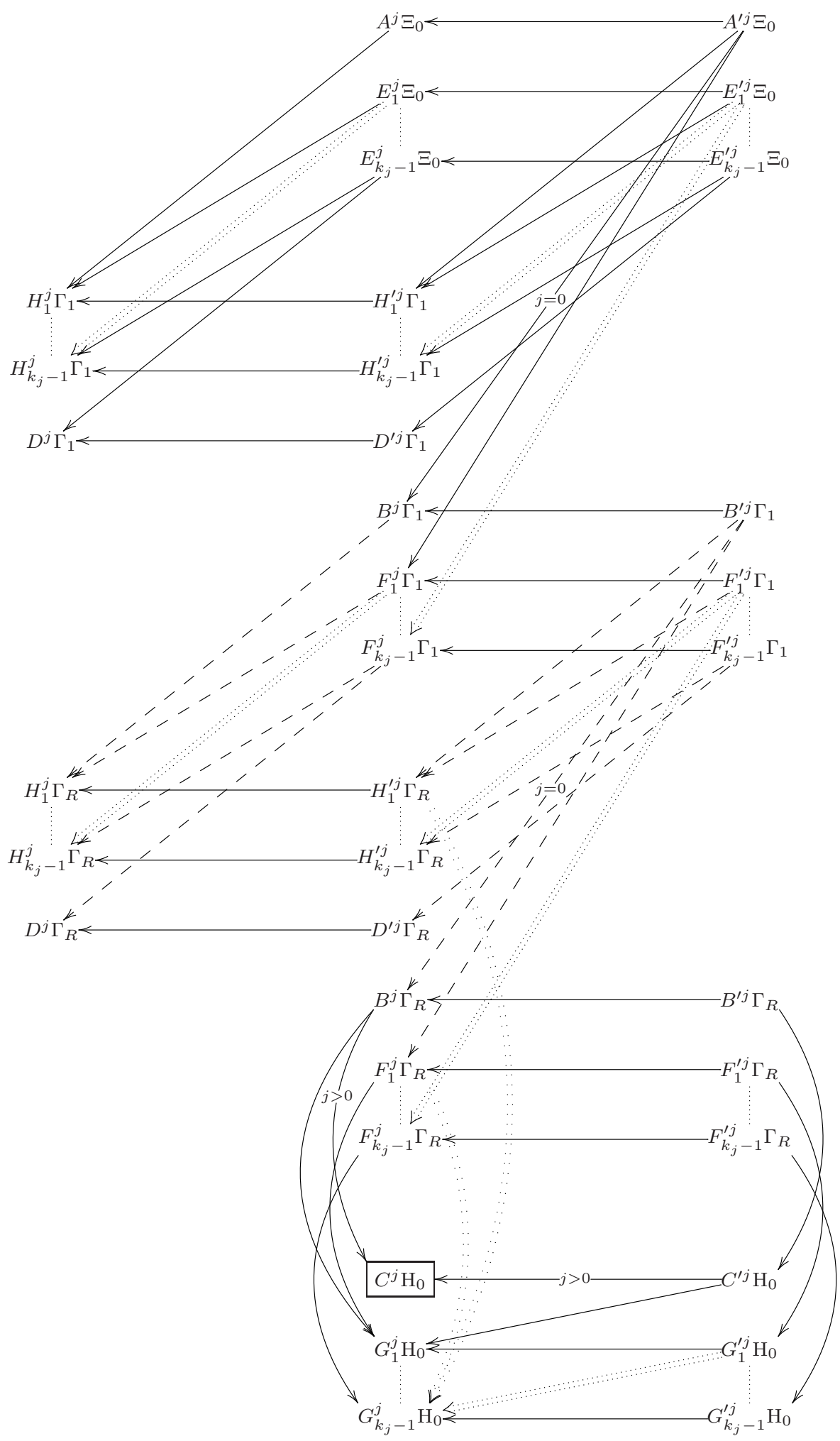

Figure 28. The subspace $Z_{\text {unst }}^{j}$ when $t>2 \tau(K)$, corresponding to the unstable chain $\Xi_{0} \stackrel{\sigma_{123}}{\longrightarrow} \Gamma_{1} \stackrel{\sigma_{23}}{\longrightarrow} \cdots \stackrel{\sigma_{23}}{\longrightarrow} \Gamma_{R} \stackrel{\sigma_{2}}{\longrightarrow} \mathrm{H}_{0}$. 


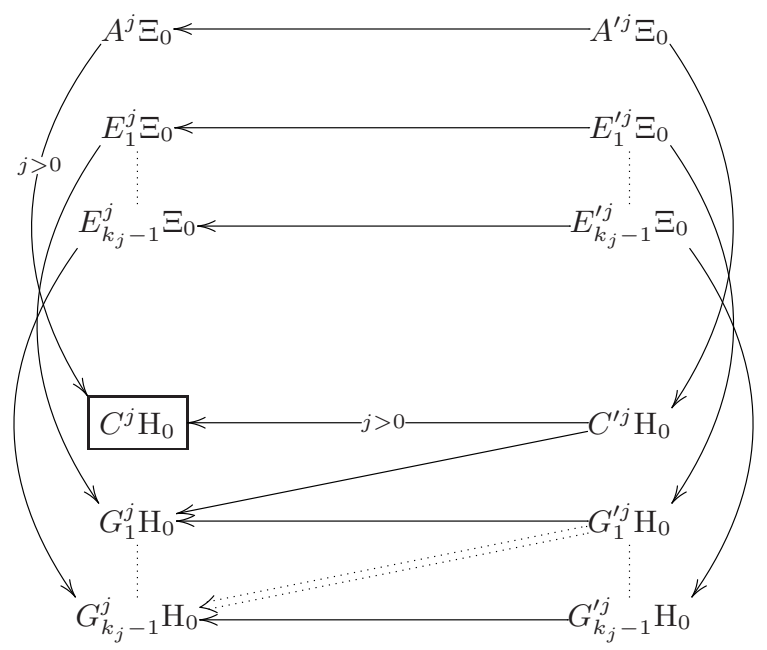

Figure 29. The subspace $Z_{\text {unst }}^{j}$ when $t=2 \tau(K)$, corresponding to the unstable chain $\Xi_{0} \stackrel{\sigma_{12}}{\longrightarrow} \mathrm{H}_{0}$.

for $j>0$. If $t>2 \tau(K)+1$, there are differentials $E_{k_{j-1}}^{\prime j} \Xi_{0} \rightarrow D^{\prime j} \Gamma_{2}$ and $F_{k_{j-1}}^{\prime j} \Gamma_{I} \rightarrow D^{\prime j} \Gamma_{I+2}$ for all $j$, and $B^{j} \Gamma_{R-1} \rightarrow G_{1}^{j} \mathrm{H}_{0}$ for $j>0$.

Next, we may have some differentials that preserve the decomposition

$$
\bigoplus_{J} Z_{\text {vert }}^{J, *} \oplus \bigoplus_{J} Z_{\text {hor }}^{J, *} \oplus Z_{\text {unst }}^{*}
$$

but which come from the multiplications on $Q$ that do not preserve the splitting $Q=\bigoplus_{j=0}^{n} Q^{j}$, shown in Figures [19, 23, and 24, The resulting differentials are shown in Table 2. In each line that involves expressions like $\mathrm{K}_{I}^{J}, \Lambda_{I}^{J}$, and $\Gamma_{I}$, we assume that $K_{J}, L_{J}$, or $R$ is sufficiently large for the indices to make sense and that $I$ ranges over appropriate bounds. The symbol * denotes both primed and unprimed symbols; thus, for instance, the notation $A^{* j} \Xi_{2 J} \rightarrow D^{* h} \mathrm{~K}_{2}^{J}$ means that there are differentials $A^{j} \Xi_{2 J} \rightarrow D^{h} \mathrm{~K}_{2}^{J}$ and $A^{\prime j} \Xi_{2 J} \rightarrow D^{\prime h} \mathrm{~K}_{2}^{J}$. Additionally, note that if $k_{h}=1$, then we replace $H_{1}^{h}$ by $D^{h}$ where it appears; if $k_{j}=1$, we replace $E_{k_{j}-1}^{\prime j}, F_{k_{j}-1}^{\prime j}$, and $G_{k_{j}-1}^{\prime j}$ by $A^{\prime j}, B^{\prime j}$, and $C^{\prime j}$, respectively.

Notice that almost all of the differentials in Table 2 drop the filtration level by a nonzero amount. The two exceptions are $A^{j} \Xi_{2 J-1} \rightarrow D^{\prime h} \mathrm{~K}_{K_{J}}^{J}$ and $A^{j} \Xi_{0} \rightarrow D^{\prime h} \Gamma_{R}$ in the second column.

Finally, we must look at differentials that do not respect the splitting at all. Notice that the sequence $\sigma_{3} \sigma_{2} \sigma_{1}$ occurs several times in Figures 21] and 22, and the sequences $\sigma_{3} \sigma_{2} \sigma_{12}$ and $\sigma_{3} \sigma_{2} \sigma_{123}$ occur in Equations (4.3) and (4.5), and these are the only such sequences that appear. More precisely, in $Q^{j}$ with $k_{j}>1$, we have the following 


\begin{tabular}{|c|c|c|c|}
\hline & $u_{2 j, h}=1, j, h>0$ & $v_{2 j, h}=1, j>0$ & $v_{2 j-1, h}=1$ or $w_{h}=1$ \\
\hline$Z_{\text {vert }}^{J}$ & $\begin{aligned} A^{\prime j} \Xi_{2 J} & \rightarrow H_{1}^{h} \mathrm{~K}_{2}^{J} \\
B^{\prime j} \mathrm{~K}_{I}^{J} & \rightarrow H_{1}^{h} \mathrm{~K}_{I+1}^{J} \\
B^{\prime j} \mathrm{~K}_{I}^{J} & \rightarrow H_{1}^{h} \mathrm{~K}_{I+2}^{J}\end{aligned}$ & $\begin{aligned} A^{* j} \Xi_{2 J} & \rightarrow D^{* h} \mathrm{~K}_{2}^{J} \\
B^{* j} \mathrm{~K}_{I}^{J} & \rightarrow D^{* h} \mathrm{~K}_{I}^{J} \\
B^{* j} \mathrm{~K}_{I}^{J} & \rightarrow D^{* h} \mathrm{~K}_{I+2}^{J} \\
A^{j} \Xi_{2 J-1} & \rightarrow D^{\prime h} \mathrm{~K}_{K_{J}}^{J}\end{aligned}$ & $\begin{aligned} E_{k_{j}-1}^{\prime j} \Xi_{2 J} & \rightarrow D^{h} \mathrm{~K}_{1}^{J} \\
E_{k_{j}-1}^{\prime j} \Xi_{2 J} & \rightarrow D^{h} \mathrm{~K}_{3}^{J} \\
F_{k_{j}-1}^{\prime j} \mathrm{~K}_{I}^{J} & \rightarrow D^{h} \mathrm{~K}_{I+1}^{J} \\
F_{k_{j}-1}^{\prime j} \mathrm{~K}_{I}^{J} & \rightarrow D^{h} \mathrm{~K}_{I+3}^{J}\end{aligned}$ \\
\hline$Z_{\text {hor }}^{J}$ & 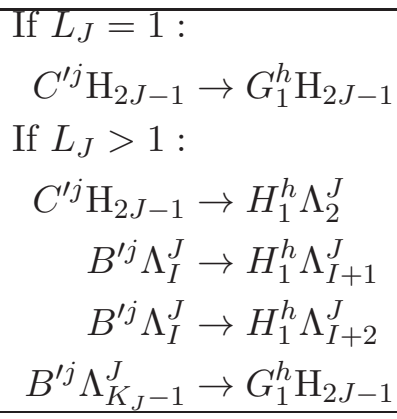 & $\begin{aligned} C^{* j} \mathrm{H}_{2 J-1} & \rightarrow D^{* J} \Lambda_{1}^{J} \\
C^{* j} \mathrm{H}_{2 J-1} & \rightarrow D^{* J} \Lambda_{2}^{J} \\
B^{* j} \Lambda_{I}^{J} & \rightarrow D^{* h} \Lambda_{I}^{J} \\
B^{* j} \Lambda_{I}^{J} & \rightarrow D^{* h} \Lambda_{I+2}^{J}\end{aligned}$ & $\begin{aligned} G_{k_{j}-1}^{\prime j} \mathrm{H}_{2 J-1} & \rightarrow D^{h} \Lambda_{1}^{J} \\
G_{k_{j}-1}^{\prime j} \mathrm{H}_{2 J-1} & \rightarrow D^{h} \Lambda_{3}^{J} \\
F_{k_{j}-1}^{\prime j} \Lambda_{I}^{J} & \rightarrow D^{h} \Lambda_{I+1}^{J} \\
F_{k_{j}-1}^{\prime j} \Lambda_{I}^{J} & \rightarrow D^{h} \Lambda_{I+3}^{J}\end{aligned}$ \\
\hline $\begin{array}{c}Z_{\text {unst }} \\
t<2 \tau(K)\end{array}$ & $\begin{array}{l}C^{\prime j} \mathrm{H}_{0} \rightarrow H_{1}^{h} \Gamma_{2} \\
B^{\prime j} \Gamma_{I} \rightarrow H_{1}^{h} \Gamma_{I+1} \\
B^{\prime j} \Gamma_{I} \rightarrow H_{1}^{h} \Gamma_{I+2}\end{array}$ & $\begin{aligned} C^{* j} \mathrm{H}_{0} & \rightarrow D^{* J} \Gamma_{1} \\
C^{* j} \mathrm{H}_{0} & \rightarrow D^{* J} \Gamma_{2} \\
B^{* j} \Gamma_{I} & \rightarrow D^{* h} \Gamma_{I} \\
B^{* j} \Gamma_{I} & \rightarrow D^{* h} \Gamma_{I+2} \\
A^{j} \Xi_{0} & \rightarrow D^{\prime h} \Gamma_{R}\end{aligned}$ & $\begin{aligned} G_{k_{j}-1}^{\prime j} \mathrm{H}_{0} & \rightarrow D^{h} \Gamma_{1} \\
G_{k_{j}-1}^{\prime j} \mathrm{H}_{0} & \rightarrow D^{h} \Gamma_{3} \\
F_{k_{j}-1}^{\prime j} \Gamma_{I} & \rightarrow D^{h} \Gamma_{I+1} \\
F_{k_{j}-1}^{\prime j} \Gamma_{I} & \rightarrow D^{h} \Gamma_{I+3}\end{aligned}$ \\
\hline $\begin{array}{c}Z_{\text {unst }}, \\
t>2 \tau(K)\end{array}$ & $\begin{array}{l}\text { If } R=1: \\
A^{\prime j} \Xi_{0} \rightarrow G_{1}^{h} \mathrm{H}_{0} \\
\text { If } R>1: \\
A^{\prime j} \Xi_{0} \rightarrow H_{1}^{h} \Gamma_{2} \\
B^{\prime j} \Gamma_{I} \rightarrow H_{1}^{h} \Gamma_{I+1} \\
B^{\prime j} \Gamma_{I} \rightarrow H_{1}^{h} \Gamma_{I+2} \\
B^{\prime j} \Gamma_{R-1} \rightarrow G_{1}^{h} \mathrm{H}_{0}\end{array}$ & $\begin{array}{l}A^{* j} \Xi_{0} \rightarrow D^{* h} \Gamma_{2} \\
B^{* j} \Gamma_{I} \rightarrow D^{* h} \Gamma_{I} \\
B^{* j} \Gamma_{I} \rightarrow D^{* h} \Gamma_{I+2}\end{array}$ & $\begin{aligned} E_{k_{j}-1}^{\prime j} \Xi_{0} & \rightarrow D^{h} \Gamma_{1} \\
E_{k_{j}-1}^{\prime j} \Xi_{0} & \rightarrow D^{h} \Gamma_{3} \\
F_{k_{j}-1}^{\prime j} \Gamma_{I} & \rightarrow D^{h} \Gamma_{I+1} \\
F_{k_{j}-1}^{\prime j} \Gamma_{I} & \rightarrow D^{h} \Gamma_{I+3}\end{aligned}$ \\
\hline
\end{tabular}

TABLE 2. Differentials arising from the multiplications in Figures 19, 23, and 24.

multiplications:

$$
\begin{aligned}
& A^{j} \stackrel{\sigma_{3} \sigma_{2} \sigma_{1}}{\longrightarrow} H_{1}^{j} \quad A^{\prime j} \stackrel{\sigma_{3} \sigma_{2} \sigma_{1}}{\longrightarrow} H_{1}^{\prime j} \\
& E_{i}^{j} \stackrel{\sigma_{3} \sigma_{2} \sigma_{1}}{\longrightarrow} H_{i+1}^{j} \quad E_{i}^{\prime j} \stackrel{\sigma_{3} \sigma_{2} \sigma_{1}}{\longrightarrow} H_{i+1}^{\prime j} \quad\left(i=1, \ldots, k_{j}-2\right) \\
& E_{k_{j}-1}^{j} \stackrel{\sigma_{3} \sigma_{2} \sigma_{1}}{\longrightarrow} D^{j} \quad E_{k_{j}-1}^{\prime j} \stackrel{\sigma_{3} \sigma_{2} \sigma_{1}}{\longrightarrow} D^{\prime j} \\
& A^{\prime j} \stackrel{\sigma_{3} \sigma_{2} \sigma_{12}}{\longrightarrow} G_{2}^{j} \quad A^{\prime j} \stackrel{\sigma_{3} \sigma_{2} \sigma_{123}}{\longrightarrow} H_{2}^{j} \\
& E_{i}^{\prime j} \stackrel{\sigma_{3} \sigma_{2} \sigma_{12}}{\longrightarrow} G_{i+2}^{j} \quad E_{i}^{\prime j} \stackrel{\sigma_{3} \sigma_{2} \sigma_{123}}{\longrightarrow} H_{i+2}^{j} \quad\left(i=1, \ldots, k_{j}-3\right) \\
& E_{k_{j}-2}^{\prime j} \stackrel{\sigma_{3} \sigma_{2} \sigma_{123}}{\longrightarrow} D^{j}
\end{aligned}
$$


If $k_{j}=1$, then we simply have $A^{j} \stackrel{\sigma_{3} \sigma_{2} \sigma_{1}}{\longrightarrow} D^{j}$ and $A^{\prime j} \stackrel{\sigma_{3} \sigma_{2} \sigma_{1}}{\longrightarrow} D^{\prime j}$. Finally, from Figure 23. if $v_{2 j, h}=1$, then there are multiplications $A^{j} \stackrel{\sigma_{3} \sigma_{2} \sigma_{1}}{\longrightarrow} D^{h}$ and $A^{\prime j} \stackrel{\sigma_{3} \sigma_{2} \sigma_{1}}{\longrightarrow} D^{\prime h}$.

Notice that all of these multiplications come out of $A^{j}, A^{\prime j}, E_{i}^{j}$, or $E_{i}^{\prime j}$, all of which are paired with $\left\{\Xi_{0}, \ldots, \Xi_{2 N}\right\}$ rather than $\left\{\mathrm{H}_{0}, \ldots, \mathrm{H}_{2 N}\right\}$ in (4.12). It follows that each group $Z_{\text {hor }}^{J, *}$ is actually a direct summand as a chain complex. We shall see that the generator of the total homology comes from $\bigoplus_{J} Z_{\text {vert }}^{J, *} \oplus Z_{\text {unst }}^{*}$, so we may ignore each of the $Z_{\text {hor }}^{J, *}$ summands as before. Furthermore, if we define $U_{P, M}, V_{P, M}$, and $W_{P}$ analogously to $u_{p, h}, v_{p, m}$, and $w_{p}$ above, then we obtain differentials from $A^{j} \Xi_{P}$, $A^{\prime j} \Xi_{P}, E_{i}^{j} \Xi_{P}$, and/or $E_{i}^{\prime j} \Xi_{P}$ to elements of $Z_{\mathrm{vert}}^{M}$ and $Z_{\text {unst }}$ whenever $U_{P, M}, V_{P, M}$, or $W_{P}$ is nonzero. Specifically:

- If $V_{P, M}=1$, then there are differentials

$$
\begin{aligned}
A^{j} \Xi_{P} & \rightarrow H_{1}^{j} \mathrm{~K}_{K_{M}}^{M} & A^{\prime j} \Xi_{P} & \rightarrow H_{1}^{\prime j} \mathrm{~K}_{K_{M}}^{M} \\
E_{i}^{j} \Xi_{P} & \rightarrow H_{i+1}^{j} \mathrm{~K}_{K_{M}}^{M} & E_{i}^{\prime j} \Xi_{P} & \rightarrow H_{i+1}^{\prime j} \mathrm{~K}_{K_{M}}^{M} \quad\left(i=1, \ldots, k_{j}-2\right) \\
E_{k_{j}-1}^{j} \Xi_{P} & \rightarrow D^{j} \mathrm{~K}_{K_{M}}^{M} & E_{k_{j}-1}^{\prime j} \Xi_{P} & \rightarrow D^{\prime j} \mathrm{~K}_{K_{M}}^{M}
\end{aligned}
$$

if $k_{j}>1$, and $A^{j} \Xi_{P} \rightarrow D^{j} \mathrm{~K}_{K_{M}}^{M}$ and $A^{\prime j} \Xi_{P} \rightarrow D^{\prime j} \mathrm{~K}_{K_{M}}^{M}$ if $k_{j}=1$. Also, if $v_{2 j, h}=1$, then there are differentials $A^{j} \Xi_{P} \rightarrow D^{h} \mathrm{~K}_{K_{M}}^{M}$ and $A^{\prime j} \Xi_{P} \rightarrow D^{\prime h} \mathrm{~K}_{K_{M}}^{M}$.

Similarly, if $W_{P}=1$ and $t<2 \tau(K)$, then we obtain similar differentials going into $Z_{\text {unst }}$, replacing $\mathrm{K}_{K_{M}}^{M}$ by $\Gamma_{R}$.

- If $U_{P, M}=1$, then there are differentials

$$
\begin{aligned}
A^{\prime j} \Xi_{P} & \rightarrow H_{2}^{j} \mathrm{~K}_{1}^{M} \\
E_{i}^{\prime j} \Xi_{P} & \rightarrow H_{i+2}^{j} \mathrm{~K}_{1}^{M} \quad\left(i=1, \ldots, k_{j}-3\right) \\
E_{k_{j}-2}^{\prime j} \Xi_{P} & \rightarrow D^{j} \mathrm{~K}_{1}^{M} .
\end{aligned}
$$

Similarly, if $W_{P}=1$ and $t>2 \tau(K)$, then we obtain similar differentials going into $Z_{\text {unst }}$, replacing $\mathrm{K}_{1}^{M}$ by $\Gamma_{1}$.

- Finally, if $W_{P}=1$ and $t=2 \tau(K)$, there are differentials

$$
\begin{aligned}
& A^{\prime j} \Xi_{P} \rightarrow G_{2}^{j} \mathrm{H}_{0} \\
& E_{i}^{\prime j} \Xi_{P} \rightarrow G_{i+2}^{j} \mathrm{H}_{0}\left(i=1, \ldots, k_{j}-3\right)
\end{aligned}
$$

4.4. Computation of $\tau\left(D_{J, s}(K, t)\right)$. We now describe the edge cancellations that occur in each of the pieces. Recall that we must cancel edges in increasing order of the amount by which they drop filtration level. We shall see that a single generator survives. The filtration level of this generator, by definition, is $\tau\left(D_{J, s}(K, t)\right)$.

We start by canceling the filtration-preserving edges in $Z_{\mathrm{vert}}^{J, j}$. Note that there are are no other edges into $B^{\prime j} K_{K_{J}}^{J}$ or $F_{i}^{\prime j} \mathrm{~K}_{K_{J}}^{J}$, so eliminating the edges coming from these does not introduce any new edges. If $V_{2 J-1}, M=1$, or if $W_{2 J-1}=1$ and $t<2 \tau(K)$, then canceling the edges $A^{j} \Xi_{2 J-1} \rightarrow B^{j} \mathrm{~K}_{K_{J}}^{J}$ and $E_{i}^{j} \rightarrow F_{k}^{j} \mathrm{~K}_{K_{J}}^{J}$ introduces some new edges, which all reduce filtration level by 2 . Note also that the filtration-preserving 
edges $A^{j} \Xi_{2 j-1} \rightarrow D^{\prime h} \mathrm{~K}_{K_{J}}^{J}(j>0)$ in Table 2 are eliminated, since $B^{j} \mathrm{~K}_{K_{j}}^{J}$ has no other incoming edges when $j>0$.

In $Z_{\text {unst }}^{j}$, when $t<2 \tau(K)$, we perform the same cancellations as in $Z_{\text {vert }}^{J, j}$, mutatis mutandis. When $t>2 \tau(K)$, there are $2 k_{j}$ filtration-preserving edges to cancel when $j>0$ (namely, $B^{* j} \Gamma^{R} \rightarrow C^{* j} \mathrm{H}_{0}$ and $F_{i}^{* j} \Gamma^{R} \rightarrow G_{i}^{* j} \mathrm{H}_{0}$ for $i=1, \ldots, k_{j}-1$ ), but only $2 k_{0}-1$ such edges in $Z_{\text {unst }}^{0}$, since the generator $C_{0} \mathrm{H}_{0}$ does not exist. Thus, the generator $B^{0} \Gamma^{R}$ survives after these cancellations. Also, note that canceling $B^{j} \Gamma^{R} \rightarrow C^{j} \mathrm{H}_{0}$ and $F_{i}^{j} \Gamma^{R} \rightarrow G_{i}^{j} \mathrm{H}_{0}$ may introduce some new differentials using the arrows in Table 2, but they all filtration level by 2 .

When $t=2 \tau(K)$, the only generator in $Z_{\text {unst }}^{0}$ that survives is $A^{0} \Xi_{0}$. Notice, however, that by (4.15), there is a differential $A^{0} \Xi_{0} \rightarrow H_{1}^{0} \mathrm{~K}_{K_{M}}^{M}$ for any $M$ with $V_{0, M}=1$. All the generators of $Z_{\text {unst }}^{j}$ for $j>0$ are canceled.

We have now canceled all edges that preserve the filtration level, so we now begin canceling differentials that drop filtration level by 1 . Specifically, starting at the top of Figure 25 and working down, we cancel every edge of the form $X^{\prime} \rightarrow X$, where $X^{\prime}$ and $X$ are two generators in the same row (e.g., $A^{\prime j} \Xi_{2 J} \rightarrow A^{j} \Xi_{2 J}$ ). We use the following key observations:

- If $X$ is in filtration level 0 and $X^{\prime}$ is in level 1 , then $X$ has no other incoming edges, since by induction we have already eliminated everything above $X$ and $X^{\prime}$, and Table 2 and Equations (4.15) and (4.16) contain no differentials that go into $A^{j} \Xi_{2 J}, E_{i}^{j} \Xi_{2 J}, B^{j} \mathrm{~K}_{I}^{J}$, or $F_{i}^{j} \mathrm{~K}_{I}^{J}$ from elsewhere.

- If $X$ is in filtration level -1 and $X^{\prime}$ is in level 0 , then $X^{\prime}$ has no other outgoing edges, since Table 2 and Equations (4.15) and (4.16) contain no differentials that go out of $H_{i}^{\prime j} \mathrm{~K}_{I}^{J}$ or $D^{\prime j} \mathrm{~K}_{I}^{J}$.

Thus, we can completely cancel $Z_{\text {vert }}^{J, j}$.

If $t=2 \tau(K)$, we have now eliminated all generators except $A^{0} \Xi_{0}$, which is in filtration level 0 , so $\tau\left(D_{J_{s}}(K, t)\right)=0$ when $s<2 \tau(J)$ and $t=2 \tau(K)$.

If $t>2 \tau(K)$, we proceed with $Z_{\text {unst }}^{j}$ just as with $Z_{\text {vert }}^{J, j}$. When $j>0$, all generators in $Z_{\text {unst }}^{j}$ cancel; when $j=0$, the one surviving generator is $B^{0} \Gamma_{R}$, which is in filtration level 0. Thus, $\tau\left(D_{J_{s}}(K, t)\right)=0$ when $s<2 \tau(J)$ and $t>2 \tau(K)$.

If $t<2 \tau(K)$, when $j>0$, we start by canceling $C^{\prime j} \mathrm{H}_{0} \rightarrow C^{j} \mathrm{H}_{0}$ and proceeding downward in Figure 27, as before, eliminating all generators. When $j=0$, we start by canceling $G_{1}^{\prime j} \mathrm{H}_{0} \rightarrow G_{1}^{j} \mathrm{H}_{0}$ and proceed downward, and we thus see that the only surviving generator is $C^{\prime j} \mathrm{H}_{0}$, which is in filtration level 1 . Thus, $\tau\left(D_{J_{s}}(K, t)\right)=1$ when $s<2 \tau(J)$ and $t<2 \tau(K)$.

Finally, we must return to the case where $s=2 \tau(J)$. Recall that $Q_{0}$ in this case consists of three generators, all in filtration level 0 , as in (4.11). For $j>0$, the definitions of $Z_{\mathrm{vert}}^{J, j}, Z_{\mathrm{hor}}^{J, j}$, and $Z_{\text {unst }}^{j}$ go through the same way, and we see again that all of the resulting generators eventually cancel. It follows that the surviving generator must be in filtration level 0 , so $\tau\left(D_{J, s}(K, t)\right)=0$ whenever $s=2 \tau(K)$. 


\section{Other Results Regarding $D_{J, s}(K, t)$}

Prior to Hedden's complete computation of $\widehat{\mathrm{HFK}}$ and $\tau$ of all twisted Whitehead doubles [4], Livingston and Naik [13] used the formal properties of $\tau$ to understand the asymptotic behavior of $\tau$ for large values of the twisting parameter. They proved:

Theorem 5.1. Suppose $\nu$ is any homomorphism from the smooth knot concordance group to $\mathbb{Z}$ with the properties that $|\nu(K)| \leq g_{4}(K)$ and $\nu\left(T_{p, q}\right)=(p-1)(q-1) / 2$, where $p, q>0$ and $T_{p, q}$ denotes the $(p, q)$ torus knot. Then for any knot $K$, there exists $t_{\nu}(K) \in \mathbb{Z}$ such that

$$
\nu\left(W h_{+}(K, t)\right)= \begin{cases}1 & t \leq t_{\nu}(K) \\ 0 & t>t_{\nu}(K)\end{cases}
$$

and $T B(K) \leq t_{\nu}(K)<-T B(-K)$ (where $T B(K)$ denotes the maximal ThurstonBennequin number of $K)$.

Two invariants satisfying the hypotheses of Theorem 5.1 are $\tau(K)$ and $-s(K) / 2$, a renormalization of Rasmussen's concordance invariant $s(K)$ [17]. Around the same time, Hedden and Ording [5] proved that these two invariants are not equal by showing that $\tau\left(W h_{+}\left(T_{2,3}, 2\right)\right)=0$ while $s\left(W h_{+}\left(T_{2,3}, 2\right)\right)=-2$, disproving a conjecture of Rasmussen. Later, Hedden [4 showed that $t_{\tau}(K)=2 \tau(K)-1$ for any knot $K$. Finding a general formula for the $s$ invariant of Whitehead doubles remains an open question.

We may extend the techniques of Livingston and Naik to study knots of the form $D_{J, s}(K, t)$ as well.

Proposition 5.2. Let $\nu$ be an invariant satisfying the hypotheses of Theorem [5.1, and fix knots $J$ and $K$.

(1) If $s \leq T B(J)$ and $t \leq T B(K)$, then $\nu\left(D_{J, s}(K, t)\right)=1$. If $s \geq-T B(-J)$ and $t \geq-T B(-K)$, then $\nu\left(D_{J, s}(K, t)\right)=-1$.

(2) For fixed $s$ (resp. $t)$, the function $t \mapsto \nu\left(D_{J, s}(K, t)\right)$ (resp. $s \mapsto \nu\left(D_{J, s}(K, t)\right)$ ) is non-increasing and has as its image either $\{-1,0\},\{0\}$, or $\{0,1\}$.

Proof. The proof is very similar to that of [13, Theorem 2].

Let $A(J, s)$ denote an annulus in $S^{3}$, embedded along $J$ with framing $s$, and define $A(K, t)$ analogously. We may obtain a Seifert surface for $D_{J, s}(K, t)$ as a plumbing $A(J, s) * A(K, t)$. By results of Rudolph [19, 21], when $s \leq T B(J)$ and $t \leq T B(K)$, the annuli $A(J, s)$ and $A(K, t)$ are quasipositive surfaces, so $A(J, s) * A(K, t)$ is also a quasipositive surface. Thus, $D_{J, s}(K, t)$ is a strongly quasipositive knot with genus 1 , and hence $\nu\left(D_{J, s}(K, t)\right)=1$ [12, Theorem 4]. Mirroring gives the second half of $(1)$.

The non-increasing statement in (2) follows from the fact that $D_{J, s}(K, t)$ is obtained from $D_{J, s-1}(K, t)$ or $D_{J, s}(K, t-1)$ by changing a positive crossing to a negative crossing, which can only preserve or decrease $\nu$ [12, Corollary 3]. Also, since $D_{J, s}(K, t)$ is related to $D_{J, s^{\prime}}(K, t)$ or $D_{J, s}\left(K, t^{\prime}\right.$ ) (for any $s^{\prime}$ or $t^{\prime}$ ) by changing the number of twists in a band of a Seifert surface, each of the two functions can assume at most two values, either $\{-1,0\}$ or $\{0,1\}[13$, Corollary 5]. Finally, we rule out the possibility that either 

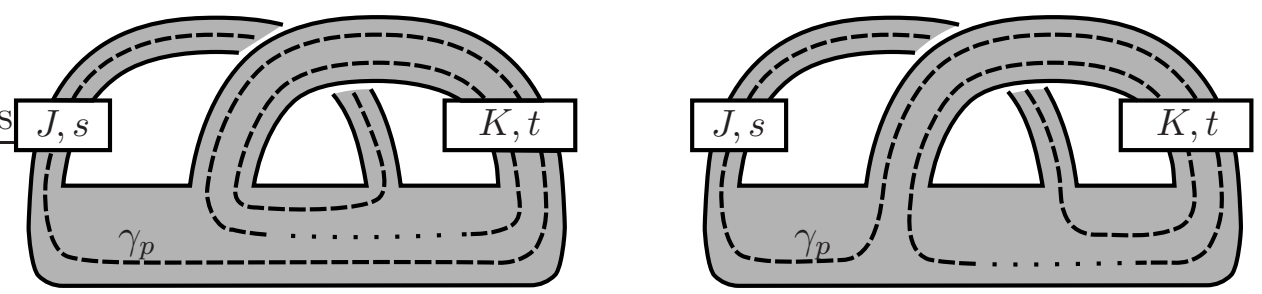

FigurE 30. The Seifert surface $F$ with the curve $\gamma_{p}$, in the cases where $p<0$ (left) and $p>0$ (right).

of the functions in (1) is constant and nonzero. Suppose, without loss of generality, that $\nu\left(D_{J, s}(K, t)\right)=1$ for a fixed $s$ and all $t$. In particular, $\nu\left(D_{J, s}(K,-T B(-K))\right)=$ 1. On the other hand, $\nu\left(D_{J,-T B(-J)}(K,-T B(-K))\right)=-1$, which contradicts the fact that the image of the function $s \mapsto \nu\left(D_{J, s}(K,-T B(-K))\right)$ contains at most two consecutive integers.

It follows that if $s \leq T B(J)$ and $t \geq-T B(-K)$, or $s \geq-T B(-J)$ and $t \leq T B(K)$, then $\nu\left(D_{J, s}(K, t)\right)=0$. Thus, for large absolute values of $s$ and $t, \nu\left(D_{J, s}(K, t)\right)=$ $\tau\left(D_{J, s}(K, t)\right)$. On the other hand, the behavior of $\nu\left(D_{J, s}(K, t)\right.$ for small $s$ and $t$ (specifically, when $T B(J)<s<-T B(-J)$ or $T B(K)<t<-T B(-K)$ ) may be more complicated than the simple behavior of $\tau$ given by Theorem 1.1 .

In another direction, we may also look for instances when $D_{J, s}(K, t)$ is actually smoothly slice. The following proposition generalizes Casson's argument [7, page 227] that the $p(p+1)$-twisted positive Whitehead double of the $(p, p+1)$ torus knot is smoothly slice. For an oriented knot $K$ and relatively prime integers $p, q$, let $C_{p, q}(K)$ denote the $(p, q)$-cable of $K$. (Note that $C_{p, q}(K)^{r}=C_{-p,-q}(K)=C_{p, q}\left(K^{r}\right)$ and $\overline{C_{p, q}(K)}=C_{p,-q}(\bar{K})$.)

Proposition 5.3. Let $K$ be any knot, and let $p, t \in \mathbb{Z}$. If $J$ is any knot that is smoothly concordant to $-C_{p, p t \pm 1}(K)$, then $D_{J,-p(p t \pm 1)}(K, t)$ is smoothly slice.

Proof. Let $F$ be the Seifert surface for $D_{J, s}(K, t)$ shown in Figure 30, and let $\gamma_{p}$ be a curve that winds once around the band tied into $J$ and $p$ times around the band tied into $K$, as indicated. The knot type of $\gamma_{p}$ is $C_{p, p t+1}(K)$, and the surface framing on $\gamma_{p}$ is $s+p+p^{2} t$. Thus, if $J$ is smoothly concordant to $-C_{p, p t+1}(K)$ and $s=-p(p t+1)$, we may surger $F$ along $\gamma_{p}$ in $D^{4}$ along a smooth slice disk for $J \# C_{p, p t+1}(K)$, resulting in a smooth slice disk for $D_{J, s}(K, t)$.

If we reverse the crossing between the two bands of $F$, we obtain the result with the opposite signs.

Proposition 5.3 is quite interesting in light of work of Hom [6], who found a general formula for the $\tau$ invariant of all cable knots in terms of $p, q, \tau(K)$, and an invariant $\epsilon(K) \in\{-1,0,1\}$ that depends solely on the knot Floer complex of $K$. She proved:

Theorem 5.4. Let $K$ be a knot, and let $p>0$. Then:

- If $\epsilon(K)=1$, then $\tau\left(C_{p, q}(K)\right)=p \tau(K)+\frac{1}{2}(p-1)(q-1)$ for all $q$.

- If $\epsilon(K)=-1$, then $\tau\left(C_{p, q}(K)\right)=p \tau(K)+\frac{1}{2}(p-1)(q+1)$ for all $q$. 
- If $\epsilon(K)=0$, then $\tau(K)=0$, and

$$
\tau\left(C_{p, q}(K)\right)= \begin{cases}\frac{1}{2}(p-1)(q+1) & q<0 \\ \frac{1}{2}(p-1)(q-1) & q>0 .\end{cases}
$$

We may use Theorem 5.4 to compute the value of $\tau$ for the cable knots appearing in Proposition 5.3, where we take $t=2 \tau(K)$.

Corollary 5.5. For any knot $K$, if either $\epsilon(K) \geq 0$ and $p>0$, or $\epsilon(K) \leq 0$ and $p<0$, there exists a knot $J$ such that $D_{J, 2 \tau(J)-p}(K, 2 \tau(K))$ is smoothly slice, while $\tau\left(D_{J, 2 \tau(J)-p}\left(K, 2 \tau(K)-\frac{p}{|p|}\right)\right) \neq 0$.

Proof. Suppose that $\epsilon(K)=1$ and $p>0$. Set $J=-C_{p, 2 p \tau(K)+1}(K)$, so that:

$$
\begin{aligned}
2 \tau(J)-p & =-2 \tau\left(C_{p, 2 p \tau(K)+1}(K)\right)-p \\
& =-2 p \tau(K)-(p-1)(2 p \tau(K))-p \\
& =-2 p^{2} \tau(K)-p \\
& =-p(2 p \tau(K)+1) .
\end{aligned}
$$

By Proposition 5.3, $D_{J, 2 \tau(J)-p}(K, 2 \tau(K))$ is smoothly slice. On the other hand, $\tau\left(D_{J, 2 \tau(J)-p}(K, 2 \tau(K)-1)=1\right.$ by Theorem 1.1. The case where $\epsilon(K)=-1$ and $p<0$ follows by mirroring, since $\epsilon(\bar{K})=-\epsilon(K)$. Finally, if $\epsilon(K)=0$, we set $J=-C_{p, 1}(K)$ if $p>0$ and $J=-C_{-p,-1}$ if $p<0$.

Theorem 1.1 says that the set $\left\{(s, t) \in \mathbb{Z}^{2} \mid D_{J, s}(K, t)=0\right\}$ always has the same shape for any $J$ and $K$, up to translation: the union of the second and fourth quadrants of the $\mathbb{Z}^{2}$ lattice, including both axes. Corollary 5.5 implies that any point on the boundary of this region may be realized by a smoothly slice knot $D_{J, s}(K, t)$ for suitable choices of $J$ and $K$.

Finally, recall that the main idea of the proof of Theorem 1.1 is that only the form of the unstable chains in $\widehat{\mathrm{CFD}}\left(\mathcal{X}_{J}^{s}\right)$ and $\widehat{\mathrm{CFD}}\left(\mathcal{X}_{K}^{t}\right)$ matters for the computation of $\tau\left(D_{J, s}(K, t)\right)$. Petkova [16] and Hom [6] have observed similar behavior in using bordered Heegaard Floer homology to compute $\tau\left(C_{p, q}(K)\right)$. The invariant $\epsilon(K)$ defined by Hom describes the structure of the part of $\widehat{\operatorname{CFD}}\left(\mathcal{X}_{K}^{t}\right)$ "near" the unstable chain. Specifically, when we take vertically and horizontally reduced bases $\left\{\tilde{\xi}_{0}, \ldots, \tilde{\xi}_{2 n}\right\}$ and $\left\{\tilde{\eta}_{0}, \ldots, \tilde{\eta}_{2 n}\right\}$ for $\mathrm{CFK}^{-}(K)$, we may arrange that $\tilde{\xi}_{0}=\tilde{\eta}_{i}$ for some $i$. The cases $\epsilon(K)=1, \epsilon(K)=-1$, and $\epsilon(K)=0$ correspond, respectively, to whether $i$ is even and positive, odd and positive, or zero. Within each case, Hom showed that only the form of the unstable chain matters for computing $\tau\left(C_{p, q}(K)\right)$. It is an interesting question whether the behavior of $\tau$ for more general classes of satellite knots can be described in this way.

\section{Appendix A. Notes on the COMPUtation}

This section provides further details about the Mathematica notebooks used for the computations in Section 3. The computation makes use of two packages that are designed to be useful for Heegaard Floer homology computations: HeegaardDiagram.nb, 
which is used to find the index-1 domains in a Heegaard diagram, and TorusAlgebra.nb, which provides algebraic tools for working with $\mathcal{A}_{\infty}$-modules and type- $D$ structures over the torus algebra. In the hope that these tools will be of use to other researchers in the future, Sections A.1 and A.2 provide brief user's guides. The computation of $\widehat{\mathrm{CFAA}}\left(\mathcal{Y}, B_{3}, 0\right)$ is found in the notebook Borromean.nb, which is described in Section A.3. All of these notebooks are available as ancillary materials in this article's arXiv folder: http://arxiv.org/src/1008.3349/anc.

A.1. HeegaardDiagram.nb. The file HeegaardDiagram.nb contains functions for finding all of the positive domains of index 1 in a Heegaard diagram in order to compute $\widehat{\mathrm{CF}}$ of a closed 3-manifold or $\widehat{\mathrm{CFD}}$ of a bordered manifold with all boundary components of genus 1 . This is fundamentally a problem of solving systems of linear equations. Obviously, the program does not determine whether or not a given domain supports holomorphic representatives, but it generates a list of domains whose moduli spaces that can then be checked by hand (or with computer assistance, as in the present setting).

Preliminary input. The basic input for the program consists of the following data. We label all of the intersection points between the $\alpha$ and $\beta$ curves $x_{1}, \ldots, x_{n}$, and the regions of the diagram $R_{1}, \ldots, R_{m}$, so that the basepointed region is $R_{1}$. Assume the Heegaard diagram has $k$ boundary components and that the genus is $g$. (Typically $k \in\{0,1,2\}$, but in principle we can take $k$ to be arbitrary.)

To input the Heegaard diagram, one must specify the following data:

- pointdata is an $n \times 4$ array recording which regions are incident to the intersection points. Specifically, if we draw a neighborhood of $x_{i}$ such that the $\alpha$ curve is the horizontal axis, the $\beta$ curve is the vertical axis, and the four quadrants are $R_{a}, R_{b}, R_{c}$, and $R_{d}$ (starting with the upper-right quadrant and going counterclockwise), then the $i^{\text {th }}$ entry of pointdata is the list $\{a, b, c, d\}$.

- euler is a list of the Euler measures of the regions $R_{1}, \ldots, R_{m}$.

- boundary is a $k \times 3$ array that records which regions abut each boundary component of the diagram. For a closed diagram, this is simply the empty list. For a bordered diagram, if the three regions (other than $R_{1}$ ) adjacent to the $i^{\text {th }}$ boundary component are $R_{a}, R_{b}$, and $R_{c}$ - adjacent to the $\rho_{1}, \rho_{2}, \rho_{3}$ arcs, respectively, following the labeling convention for $\widehat{\mathrm{CFD}}$ - then the $i^{\text {th }}$ entry of boundary is $\{a, b, c\}$.

- alphaarcs is a $k \times 2$ array of lists recording which intersection points are on each of the $\alpha$ arcs. Specifically, if $\alpha_{1}^{i}$ and $\alpha_{2}^{i}$ are the arcs that abut the $i^{\text {th }}$ boundary component, where $\alpha_{1}^{i}$ meets the boundary between the basepoint and $\rho_{1}$, and between $\rho_{2}$ and $\rho_{3}$, the $(i, 1)^{\text {th }}$ (resp. $(i, 2)^{\text {th }}$ ) entry of alphaarcs is the list of the indices of the intersection points on $\alpha_{1}^{i}$ (resp. $\left.\alpha_{2}^{i}\right)$.

- alphacircles is a list of length $g-k$ whose $i^{\text {th }}$ entry is the list of the indices of the intersection points on the $i^{\text {th }} \alpha$ circle.

- beta is a list of length $g$ whose $i^{\text {th }}$ entry is the list of the indices of the intersection points on the $g^{\text {th }} \beta$ circle. 
The first command to execute is Initialize[], which initializes the values of several other variables that are used throughout the computation.

A generator $\left(x_{i_{1}}, \ldots, x_{i_{g}}\right)$, where $x_{i} \in \beta_{i}$, is represented by the ordered $g$-tuple of indices $\left(i_{1}, \ldots, i_{g}\right)$. Note that the points should be written in the same order as the $\beta$ circles. The list of all generators should be stored as generators, which Initialize [] does automatically (using the function FindGenerators []), but one can also define such a list manually.

Finding domains. The following functions are used in finding domains:

- PositiveDomain[from, to, constraints] takes three arguments: two generators from and to, given in the format described above, and a list constraints indicating the constraints imposed on the multiplicities of certain regions. The latter is a list of pairs $\left(a_{i}, b_{i}\right)$, where each pair corresponds to requiring the region $R_{a_{i}}$ to have multiplicity $b_{i}$. We always require that there are enough constraints so that there is at most one solution to the linear equations (2.3) . (If there are no provincial periodic domains in the diagram, then constraining the multiplicities of each of the boundary regions is sufficient; compare Lemma 3.2.) PositiveDomain[from, to, constraints] returns a list of length $m$ consisting of the multiplicities of each of the regions in the unique positive domain from from to to if one exists, and \{\} otherwise.

- Index1Domain[from, to, constraints] likewise returns the unique index1 positive domain from from to to if one exists, and \{\} otherwise. Note: Because of the way the index of a domain in a bordered diagram is computed (see page 24), this function only works properly if the multiplicity of each boundary region is either 0 or 1 . By Proposition 2.1, we need only consider such domains in order to compute $\widehat{\mathrm{CFD}}$.

- CoeffsToList[domain] takes as its argument a positive domain domain in the format output by PositiveDomain and Index1Domain as above, and it outputs the list of which regions have nonzero multiplicity, with repetitions for multiplicities greater than 1 . This format is more convenient for inspecting domains manually, especially when the number of regions is large. The function ListToCoeffs [domain] reverses this process.

- FindIndex1Domains [constraints] takes as its argument a list of constraints as above, and it outputs a list of triples \{from, to, domain\} consisting all of the index-1 domains satisfying the given constraints. Specifically, it applies the function Index1Domain to every pair of generators (taken from the list generators), and applies CoeffsToList to each of the outputs. Because this involves solving a large system of linear equations for each pair, it can take a long time to run.

- FastPositiveDomain, FastIndex1Domain, and FastFindIndex1Domains are more efficient versions of the functions above. The function Initialize[] generates a list of domains connecting each pair of consecutive generators in generators and finds a basis for the group of periodic domains.

FastPositiveDomain [i, j, constraints] 
finds a positive domain, if one exists, from the $i^{\text {th }}$ entry of generators to the $\mathrm{j}$ entry by a two-step process: first, it finds a "test domain" by adding together entries in the preloaded list of domains, and then it solves a system of linear equations to determine what linear combination of periodic domains, if any, can be added to the test domain to give a domain satisfying the needed constraints. This system typically involves far fewer variables than the used in PositiveDomain.

The other two functions work analogously. FastFindIndex1Domains saves additional time by considering only pairs of generators that occupy the appropriate $\alpha$ arcs to be compatible with the given constraints, as per Proposition 2.1 this feature can be disabled with the option setting TypeDOnly $\rightarrow$ False.

Important note: At present, FastFindIndex1Domains requires that all generators in generators represent the same $\operatorname{spin}^{c}$ structure - i.e., that there is a domain connecting any two generators. If there are multiple spin ${ }^{c}$ struc- $^{-}$ tures, they should each be handled separately, building the list of generators manually each time. The author plans to address this issue in a future version of the program.

- AllIndex1Domains [] finds all of the index-1 domains in the diagram. (This is the only function that a typical user needs to call.) Specifically, it cycles through all possible sets of constraints where each boundary region has multiplicity either 0 and 1 and calls FastFindIndex1Domains for each set. To impose constraints on regions other than the boundary, AllIndex1Domains can take an extra argument extraregions, a list of tuples $\left(a_{i}, b_{i}, c_{i}\right)$, where each one corresponds to letting the multiplicity of region $R_{a_{i}}$ range from $b_{i}$ to $c_{i}$; the default value of extraregions is \{\} . This option should be used when the diagram contains periodic domains, although some thought is needed to determine the appropriate bounds. The option setting MonitorProgress->True provides a progress indicator that indicates the time elapsed and which set of constraints is being considered.

A.2. TorusAlgebra.nb. The file TorusAlgebra.nb contains functions used for computations with $\mathcal{A}_{\infty}$ modules, type- $D$ structures, and bimodules over the torus algebra. It includes an implementation of the edge reduction algorithm described in Subsection [2.6. and it can compute the box tensor product. Although gradings are not discussed in this paper, the package also contains some functionality for working with the non-abelian grading on bordered Floer homology, with certain caveats described below. It makes considerable use of Mathematica's capabilities for pattern-matching and symbolic manipulation.

Algebra basics. The six Reeb elements in $\mathcal{A}\left(T^{2}\right)$ are represented by the symbols rho[1], rho[2], rho[3], rho[1,2], rho[2,3], and rho[1,2,3]. Here rho can be any function initialized with the command

rho[w_-_Integer, $\mathrm{x}_{-}$Integer, $\mathrm{y}_{-}$Integer, $\mathrm{z}_{---}$Integer $]:=0 / ; \mathrm{x}+1 \mathrm{l}=\mathrm{y}$; 
This guarantees that an expression containing non-consecutive indices (e.g. rho $[1,3]$ ) automatically becomes 0 . The package automatically initializes the commands rho, sigma, tau, and phi, along with the actual corresponding Greek letters.

Tensor products of algebra are elements denoted using the $\otimes$ symbol, which in Mathematica is typed Esc c *Esc or $\backslash$ [CircleTimes]. One may also use the CircleTimes command; thus,

CircleTimes [rho [3], rho[2]]

is the same as rho[3] $\otimes \mathrm{rho}[2]$.

We list the basic commands for working with the algebra, but the typical user does not need them.

- CollectVars sorts the entries of a tensor product of algebra elements according to which function they involve. Thus CollectVars $[r h o[2] \otimes \operatorname{sigma}[1] \otimes$ rho[1]] returns rho [2] $\otimes$ rho[1] $\otimes$ sigma[1]. It does not change the order of factors using the same function.

- Group takes two arguments: a tensor product of algebra elements and a list of which algebras are treated as type $A$. The other algebras are treated as type $D$, meaning that multiplications are evaluated. For instance:

- The expression Group $[r h o[1] \otimes r h o[2] \otimes$ sigma $[1], \quad\{r h o\}]$ returns rho [1] $\otimes$ rho [2] $\otimes$ sigma $[1]$.

- The expressions Group $[$ rho $[1] \otimes$ rho $[2] \otimes$ sigma $[1], \quad\{$ sigma $\}]$

and

Group [rho [1] $\otimes$ rho [2] $\otimes$ sigma $[1], \quad\{\}]$ both return rho $[1,2] \otimes$ sigma $[1]$, since $\rho_{1} \rho_{2}=\rho_{12}$ in $\mathcal{A}$.

- Group [rho[2] $\otimes$ rho[1] $\otimes$ sigma [1], \{sigma\}] returns 0, since $\rho_{2} \rho_{1}=0$ in $\mathcal{A}$.

- SelectPart and SelectRemaining both take as arguments a tensor product of algebra elements and the name of one of the algebras. SelectPart returns the factors that use the specified algebra; SelectRemaining returns the factors that do not use that algebra. Thus,

SelectPart [rho[1] $\otimes$ rho [2] $\otimes$ sigma [1], rho]

and

SelectRemaining $[r h o[1] \otimes r h o[2] \otimes$ sigma [1] , sigma]

both return rho[1] $\otimes$ rho [2].

The commands Group, SelectPart, and SelectRemaining all distribute over addition in their first arguments.

Modules. A module, bimodule, or even multimodule is represented by a list such as the following, which represents $\widehat{\mathrm{CFA}}$ of a solid torus with a particular framing:

SolidTorusA $=\{\{\{$ rho, 0$\}\},\{\{1\},\{2\},\{1\}\}$, $\{\{0, \operatorname{rho}[1], 1+\operatorname{rho}[1,2]\},\{0,0, \operatorname{rho}[2]\},\{0,0,0\}\}$, 


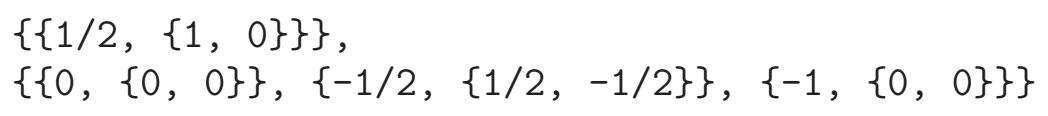

The data for a module are as follows:

- The first entry records the algebras that act and whether the action is type $D$ or type $A$. The convention is that 0 means type $A$ and 1 means type $D$. Thus, the entry $\{\{r h o, 0\}\}$ means that we have an $A$ module over a single copy of the algebra, in which elements are denoted rho[1], etc. Likewise $\{\{$ rho, 1$\},\{$ sigma, 1$\}\}$ would signify a type $D D$ bimodule, and $\{\{$ rho, 0$\}, \quad\{$ sigma, 1$\}\}$ would signify a type $A D$ bimodule in which the type $A$ action uses the rho elements and the type $D$ action uses the sigma elements. The order in which the algebras are listed matters throughout.

- The second entry records the idempotents of the generators. The convention is that 1 corresponds to $\iota_{0}$ and 2 corresponds to $\iota_{1}$. (This unfortunate convention is needed because Mathematica indexes lists from 0 rather than from 1.) In the example, the first and third generators are in idempotent $\iota_{0}$ and the second is in $\iota_{1}$. For bimodules, we record the idempotents for each action in order.

- The third entry is a matrix recording the differential or the $\mathcal{A}_{\infty}$ multiplications. The convention is that the $(i, j)^{\text {th }}$ entry records differentials from $x_{i}$ to $x_{j}$ (where $\left\{x_{1}, \ldots, x_{n}\right\}$ is a basis). Thus, in the example shown, we have:

$$
\begin{aligned}
m_{1}\left(x_{1}\right) & =x_{3} \\
m_{2}\left(x_{1}, \rho_{1}\right) & =x_{2} \\
m_{2}\left(x_{1}, \rho_{12}\right) & =x_{3} \\
m_{2}\left(x_{2}, \rho_{2}\right) & =x_{3} .
\end{aligned}
$$

For higher multiplications in a type $A$ structure, we would represent $m_{4}\left(x_{i}, \rho_{3}, \rho_{2}, \rho_{1}\right)=$ $x_{j}$, for instance, with a rho [3] $\otimes$ rho [2] $\otimes$ rho [1] term in the $(i, j)^{\text {th }}$ entry.

When working in a bimodule, we must list the elements of the two algebras in the order in which they occur in the first entry.

- The fourth and fifth entries are used when working with gradings; they may be omitted when gradings are not used. We work with the reduced grading group $G=\left\{(m ; a, b) \mid m, a, b \in \frac{1}{2} \mathbb{Z}\right)$; elements are written $\{\mathrm{m},\{\mathrm{a}, \mathrm{b}\}\}$. For bimodules, the gradings take the form $\{m,\{a, b\},\{c, d\}\}$. The fourth entry denotes the gradings of a basis for the group of periodic domains, and the fifth entry is a list of coset representatives for all of the generators.

Note: At present, gradings do not work properly for mixed bimodules (type $A D$ or $D A$ ), but they do work with single modules and with $A A$ or $D D$ bimodules.

- Extra gradings may be given as additional entries in the module. This is how we generally encode filtrations, such as the filtration on bordered Floer homology induced by a knot. 
The function DSquared can be used to verify that the differential on a type- $D$ module satisfies $\partial^{2}=0$. Note that for large matrices, this function can take a long time to run. At present, the package does not include an analogous function for verifying the $\mathcal{A}_{\infty}$ relations on a type- $A$ module.

The function CheckGradings checks whether the gradings on a module are consistent with the differential, returning either True or False. It works for single modules and $A A$ and $D D$ bimodules. It also has a FullForm option which, if enabled, lists every single nontrivial differential and whether or not it is consistent with the gradings.

Evaluating tensor products. The command for evaluating tensor products is TensorProduct. It takes four arguments: The first and third are the names of the $A$ and $D$ modules, respectively. The second argument is a number indicating which algebra on the first module is being used for the tensor product, and the fourth argument does the same for the second module.

Thus, for example, if we have two modules of the form

AABimodule $=\{\{\{r h o, 0\},\{$ sigma, 0$\}\}, \ldots\}$

DModule $=\{\{\{$ rho, 1$\}, \ldots\}$

we compute their tensor product with the command

TensorProduct [AABimodule, 1, DModule, 1]

resulting in an $A$ module of the form $\{\{\{$ sigma, 0 $\}\}, \ldots\}$. By default, the matrix in the new module will be given as a SparseArray object, unless the SparseArrayForm setting is set to False.

The variable names of the two algebras over which we are tensoring should match each other, and there should be no overlap in the remaining variable names. In the preceding example, to use the second algebra structure on AABimodule rather than the first, one might enter

TensorProduct [AABimodule /. sigma->tau, 2, DModule /. rho->tau, 1]

to change the variable names before evaluating the tensor product. Note, however, that this substitution does not work if the differentials are presented as SparseArray objects.

If either of the modules in a tensor product does not contain grading information, include the option setting Graded $\rightarrow$ False.

The following options allow the user to monitor the progress of the computation:

- MonitorProgress -> True gives running updates of which steps are being performed.

- ListGradings -> True explains the computations of the gradings of the generators in the tensor product.

- ListEdges $\rightarrow$ True explains all of the differentials that occur in the tensor product.

Each of these options is set to False by default. 
If either module has extra gradings or filtrations, you can have them extend to the tensor product using the AFiltrations or DFiltrations options. If the modules are graded, a filtration is typically the sixth entry, so we might write something like

TensorProduct [AABimodule, 1, DModule, 1, DFiltrations $\rightarrow\{6\}$ ]

to extend a filtration on DModule to the tensor product. If filtrations on both the $A$ and $D$ modules are used, those coming from the $A$ module are listed first.

Reducing modules. The ReduceModule command is used to simplify a module by looking for entries in the matrix that equal 1. The basic syntax is ReduceModule [Module], where Module is the name of the module.

If Module does not contain grading information, the option Graded should be set to False.

If Module has a filtration, you can keep track of the filtration as edges are canceled in increasing order of the amount by which they drop filtration level. If the filtration information is in the sixth entry in Module, for instance, the command is

ReduceModule[Module, Filtrations $\rightarrow\{6\}]$

The output is of the form $\{\mathrm{M},\{\{\mathrm{M} 1, \ldots, \mathrm{Mk}\}\}\}$, where $\mathrm{M}$ is the module that results from performing all simplifications, and $\mathrm{M} 1, \ldots, \mathrm{Mk}$ are the pages of the spectral sequence associated to the filtration. Usually, one is interested in the module M1, which is filtered chain homotopy equivalent to M; one can obtain this directly using the command

ReduceModule [Module, Filtrations $\rightarrow\{6\}][[2,1,1]]$

With multiple filtrations - e.g., when computing link Floer homology — ReduceModule cancels edges in the order of the amount by which they drop the total filtration level, while keeping track of each individual filtration. The syntax is, e.g.,

ReduceModule [Module, Filtrations $\rightarrow\{6,7\}]$

The option setting ListEdges -> True causes ReduceModule to display every single cancellation that is performed. The option MonitorProgress is an integer, 0 by default. When it is set to a positive number $n$, ListEdges displays an update (with the elapsed time) after every $n$ cancellations.

Examples. Several useful bordered Heegaard Floer modules are built into in the TorusAlgebra.nb package (including a few not discussed in this paper). All of these are given with gradings except where otherwise noted.

- SolidTorusA is the simple $A$ module for a solid torus with a particular framing, as described above.

- IdentityAA is the $A A$ identity bimodule.

- LHTrefoilOD and RHTrefoilOD are the $D$ modules for the complement of the two trefoils, taken with the 0-framing. 
- BorromeanDD and BorromeanAA are the filtered $D D$ and $A A$ bimodules for the complement of two components of the Borromean rings, with the filtration induced by the third component, as computed in this paper. (The Maslov gradings on BorromeanDD are computed in Borromean.nb; those on BorromeanAA were computed by hand.)

- FramingSwitchDA and FramingSwitchAA are the bimodules for the diffeomorphism of the torus that takes each slope to its perpendicular slope. For instance, the following would produce the $D$ module for $\infty$ framing on the left-handed trefoil complement:

TensorProduct [FramingSwitchDA, 2, LHTrefoil0D /. rho -> sigma, 1]

The module FramingSwitchDA currently does not contain grading information.

- SplitBasepointDD is a bimodule used for computing the link Floer homology of two-component links obtained by taking a knot in each piece of a bordered decomposition. Specifically, given nulhomologous knots $K_{1} \subset Y_{1}$ and $K_{2} \subset Y_{2}$, one would often like to be able to compute the link Floer homology of $K_{1} \cup K_{2}$ in $Y_{1} \cup Y_{2}$. However, $\widehat{\mathrm{CFA}}\left(Y_{1}, K_{1}\right) \otimes \widehat{\mathrm{CFD}}\left(Y_{2}, K_{2}\right)$ is only an invariant of the bouquet of circles obtained by connecting $K_{1}$ and $K_{2}$. The link Floer complex of the actual link is given by

$\widehat{\mathrm{CFL}}\left(Y_{1} \cup Y_{2}, K_{1} \cup K_{2}\right) \simeq \widehat{\mathrm{CFA}}\left(Y_{1}, K_{1}\right) \otimes\left(\widehat{\mathrm{CFA}}\left(Y_{2}, K_{2}\right) \otimes\right.$ SplitBasepointDD $)$

with the $\mathbb{Z} \times \mathbb{Z}$ filtration induced from the filtrations on $\widehat{\mathrm{CFA}}\left(Y_{1}, K_{1}\right)$ and $\widehat{\mathrm{CFA}}\left(Y_{2}, K_{2}\right)$. (The author is grateful to Rumen Zarev for describing this construction.)

- T42DD is the $D D$ bimodule for the exterior of the $(4,-2)$ torus link with the -2 framing on both components. If we attach a copy of the 0-framed exterior of a knot $K$ to both boundary components, we obtain the branched double cover of $W h_{+}(K)$.

A.3. Borromean.nb. We begin by loading all of the functions in HeegaardDiagram .nb and TorusAlgebra.nb packages, encoding the data of the Heegaard diagram $\mathcal{H}^{\prime}$ (Figure 8) as described above, and running Initialize []. We then run AllIndex1Domains [] and store the complete list as m1list ( $\mathrm{m}$ for Maslov). This part of the computation takes approximately 7.5 minutes to run on the author's Lenovo X220 laptop, so the output is included as a separate cell for rapid pre-loading. (In contrast, finding the domains using FindIndex1Domains [] rather than FastFindIndex1Domains [], as described in Section A.1, takes over an hour and a half.)

The next step is to partition m1list into different families of domains that share the same holomorphic geometry, saving each as a separate list and then deleting that list from m1list to ensure no repetitions. For example, as noted in Section 3.2, any index-1 domain that does not use the regions $R_{2}, R_{4}, R_{7}$, or $R_{8}$ (which are the only regions with negative Euler measure) counts for the differential. We form a list 
nicediagram consisting of all such domains and then delete them from m1list using the following commands:

nicediagram $=$ Select $[m 1$ ist, Intersection $[\#[[3]],\{2,4,7,8\}]==\{\} \&]$; m1list $=$ Complement [m1list, nicediagram $]$;

Likewise, all instances of the domains $D_{1}$ and $D_{2}$ (which count for multiple pairs of generators) can be identified as follows:

rho23cutbigons = Select [m1list, \#[[3]] ==

Sort $[J o i n[\{7,8,36,37\}$, Range $[19,30]$, Range $[49,52]]]$ \& $]$; sigma12cutbigons = Select [m1list, \#[[3]] ==

$\{4,11,17,20,24,25,29,32,35,39,43,47,50\} \quad \&]$;

m1list $=$ Complement [m1list, rho23cutbigons, sigma12cutbigons $]$;

Other families of domains with the same geometry can be identified using the command SubsetQ[1ist1, list2] (part of the HeegaardDiagram.nb package), which determines whether or not list2 is a subset of list1. For instance, we may find one family of domains with the same geometry as $D_{3}$ using the command

Select [m1list, SubsetQ[\#[[3]], $\{7,8,36,37\}] \& \&$

SubsetQ $[\{36,37,7,8,48,49,31,30,18,19,38,10,5,3,13,41,15\}, \#[[3]]] \&]$ (Compare the definition of the list rho23cutrectangles in Borromean.nb, which includes these and other domains.) After all of these families have been defined and deleted from m1list, we verify that the resulting m1list is empty, confirming that all of the domains have been classified. The reader can easily verify that every family matches up with the domains described in Section 3.1 and that the counts given in Table 1 are correct.

The domains that count for the differential (as per Proposition 3.7) are stored in the list gooddomains. We have included a verification that every composition of two domains in gooddomains either cancels against another such composition or yields a pair of algebra elements that multiply to 0 (e.g. $\rho_{2} \rho_{1}$ ), which proves that the condition (2.2) holds for $\widehat{\mathrm{CFDD}}(\mathcal{H}, 0)$.

The module $\widehat{\operatorname{CFDD}}(\mathcal{H}, 0)$ is stored as bigborromeandd (using the conventions of Section A.2). Although we have not discussed Maslov gradings anywhere in this paper, we can easily compute the relative grading between any two generators directly from the differential, so we include the gradings for future applications. The command CheckGradings [bigborromeandd] verifies that the gradings are computed correctly.

We may apply the edge-reduction algorithm to $\widehat{\operatorname{CFDD}}(\mathcal{H}, 0)$ using the ReduceModule command, as described above. The resulting module, with 19 generators, is stored as borromeandd. This step takes under a second to execute. For purely aesthetic reasons, we permute the basis elements to obtain the version described in Theorem 3.8. The matrix in the statement of that theorem is taken directly from the output of Mathematica. Note that DSquared[borromeandd] yields the zero matrix, as expected.

We then use the TensorProduct and ReduceModule comands to compute $\widehat{\operatorname{CFAD}}\left(\mathcal{Y}, B_{3}, 0\right)$ and $\widehat{\mathrm{CFAA}}\left(\mathcal{Y}, B_{3}, 0\right)$, which are respectively stored as borromeanad and borromeanaa. 
Because the grading functions in TorusAlgebra.nb do not work for mixed bimodules, we disable the Graded option for each function. The matrices in Theorem 3.9 are taken directly from the output of this computation.

\section{REFERENCES}

[1] Žarko Bižaca, An explicit family of exotic Casson handles, Proc. Amer. Math. Soc. 123 (1995), no. 4, 1297-1302.

[2] Michael H. Freedman, Whitehead 3 is a "slice" link, Invent. Math. 94 (1988), no. 1, $175-182$.

[3] Michael H. Freedman and Frank Quinn, Topology of 4-manifolds, Princeton Mathematical Series, vol. 39, Princeton University Press, Princeton, NJ, 1990.

[4] Matthew Hedden, Knot Floer homology of Whitehead doubles, Geom. Topol. 11 (2007), 22772338.

[5] Matthew Hedden and Philip Ording, The Ozsváth-Szabó and Rasmussen concordance invariants are not equal, Amer. J. Math. 130 (2008), no. 2, 441-453.

[6] Jennifer Hom, Bordered Heegaard Floer homology and the tau-invariant of cable knots, J. Topol. (2013), to appear, arXiv:1202.1463.

[7] Louis H. Kauffman, On knots, Annals of Mathematics Studies, vol. 115, Princeton University Press, Princeton, NJ, 1987.

[8] Rob Kirby (ed.), Problems in low-dimensional topology, AMS/IP Stud. Adv. Math., vol. 2, Amer. Math. Soc., Providence, RI, 1997.

[9] Adam S. Levine, Slicing mixed Bing-Whitehead doubles, J. Topol. 5 (2012), no. 3, 713-726.

[10] Robert Lipshitz, Peter Ozsváth, and Dylan Thurston, Bordered Heegaard Floer homology, arXiv:0810.0687, 2009.

[11] Bimodules in bordered Heegaard Floer homology, arXiv:1003.0598, 2010.

[12] Charles Livingston, Computations of the Ozsváth-Szabó knot concordance invariant, Geom. Topol. 8 (2004), 735-742 (electronic).

[13] Charles Livingston and Swatee Naik, Ozsváth-Szabó and Rasmussen invariants of doubled knots, Algebr. Geom. Topol. 6 (2006), 651-657 (electronic).

[14] Peter Ozsváth and Zoltán Szabó, Knot Floer homology and the four-ball genus, Geom. Topol. 7 (2003), 615-639 (electronic).

[15] _ Holomorphic disks and knot invariants, Adv. Math. 186 (2004), no. 1, 58-116.

[16] Ina Petkova, Cables of thin knots and bordered Heegaard Floer homology, Quantum Topol. 4 (2013), no. 4, 377-409.

[17] Jacob Rasmussen, Khovanov homology and the slice genus, Invent. Math. 182 (2010), no. 2, 419-447.

[18] Jacob A. Rasmussen, Floer homology and knot complements, Ph.D. thesis, Harvard University, 2003, arXiv:math/0509499.

[19] Lee Rudolph, Quasipositive annuli. (Constructions of quasipositive knots and links. IV), J. Knot Theory Ramifications 1 (1992), no. 4, 451-466.

[20] , Quasipositivity as an obstruction to sliceness, Bull. Amer. Math. Soc. (N.S.) 29 (1993), no. $1,51-59$.

[21] _ Quasipositive plumbing (constructions of quasipositive knots and links. V), Proc. Amer. Math. Soc. 126 (1998), no. 1, 257-267.

[22] Sucharit Sarkar and Jiajun Wang, An algorithm for computing some Heegaard Floer homologies, Ann. of Math. (2) 171 (2010), no. 2, 1213-1236.

Mathematics Department, Princeton University, Fine Hall, Washington Road, PRINCETON, NJ 08544

E-mail address: asl2@math.princeton.edu 